\title{
A Monte Carlo Method of Solving Heat Conduction Problems
}

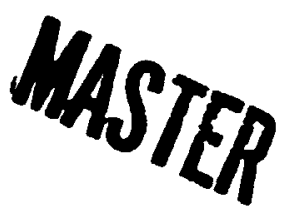

Prepared for the U.S. Nuclear Regulatory Commission Office of Nuclear Material Safety and Safeguards Under Interagency Agreement DOE 40-549-75
S. K. Fraley

T. J. Hoffman

P. N. Stevens 
OR:NL/NUREF/CSO-3

Distribution Category

UC-32

Contract No. W-7405 eng 26

\title{
A MONTE CARLO METHOD OF SOLYING HEAT CONDUCTION PROBLEAS ${ }^{\dagger}$
}

\author{
S. K. Fraley \\ T. J. Hof fman \\ Computer Sciences Divisiun \\ P. N. Stevens \\ Untversity of Tennessee
}

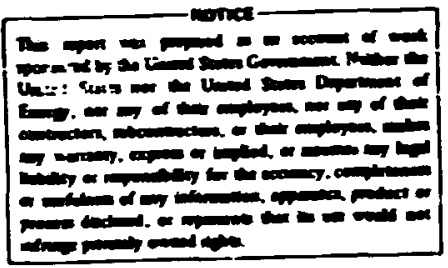

tSubmitted to the University of Tennessee by S. K. Fraley as a doctoral dissertation in the Department of Engineering Sctence and Mechantcs.

Date Puhlished: December 1977

PREPARED FGR THE U.S. NUCLEAR REGULATORY COMMISSION

OFF?CE OF NUC!EAR MATERIAL SAFETY AND SAFEGUARDS

UNDER INTERAGENCY AGREEMENT DOE 40-549-75

UNION CARBIDE CORPORATION, NUCLEAR DIVISION

operating the

Oak Ridge Gaseous Diffusion Plant - Oak Ridge Nationai Laboratory

Oak Ridge Y-12 Plant

- Paducah Gaseous Diffusion Plant

for the

DEPARTMENT OF ENERGY 


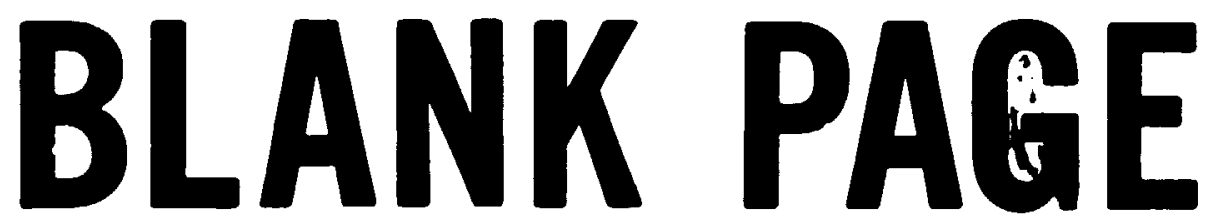


TABLE OF CONTENTS

ABSTRACT

CHAPTER

PAGE

I. INTRODUCTION ................... 1

II. TRANSPORT THEORY APPROXIMATION TO THE DIFFERENTIAL EQUATIOA OF HEAT CONDUCTION . . . . . . . . . 4

III. APPLICATION OF MONTE CARLO TO THE TRANSPORT EQUATION . . . 12

IV. COMPARISONS OF MONTE CARLO TRANSPORT SOLUTIONS TO ANALYTICAL SOLUTIONS . . . . . . . . . . . . 18

Two-Media Sphere ................. 18

Two-Media RPP Green's Function . . . . . . . . . . . 23

Two-Media RPP with Sinusoidal Interral Source... . . . 31

Two-Media RPP with Uniform Internal Source . . . . . . . 43

Two-Media RPP with Given Surface Temperature Distribution ................ . . 45

Three-Media RPP Green's Function ............. 48

Threc-Media RPP with Sinusoidal Internal Source..... 60

Two-Media RCC Green's Function ............. 64

Two-Media RCC with Functional Internal Source...... 71

Two-Media RCC with Uniform Internal Source . . . . . . 74

Two-Media RCC with Given Surface Temperature Distribution .................. 78

Two-Media RCC with Functional Internal Source and Given Surface Temperatire Distribution ....... 81

Sphere with Time Derendent Source . . . . . . . . . . . 83

Sphere with Time Dependent Source and Convective Boundary Condition.............. 84

V. CORRECTIONS TO THE TRANSPORT EQIATION APPROXIMATION . . . 90

VI. MONTE CARLO SOLUTION OF A COUPLED GAMAA RADIATION AND HEAT CONDUCTION PROBLEM . . . . . . . . . . . 98

VII. CONCLUSIONS AND RECOMMENDATIONS . . . . . . . . . . . 111

BIBLIOGRAPHY . . . . . . . . . . . . . . . . 115

APPENDIXES . . . . . . . . . . . . . . . . . . 121

A. VERIFICATION OF ANALYTJCAL SOLUTIONS . . . . . . . . . 124

B. DERIVATION OF A BOUNDED NEXT EVENT ESTIMATOR . . . . . 147 
PAGE

C. LISTING OF COAPUTER INPUT DATA AND SELECTED OUTPUT - . . - 151 


\section{LIST OF TABLES}

TABLE

PAGE

1. Temperature Comparisons for a Two-Media Sphere with a

Point Source ................. 22

2. Temperature Comparisons for a Two-Media RPP with a Sinusoidal Internal Source Using the Forward Formulation ................ . . 37

3. Temperatures at a Point Using a Bounded Next Event Estimator . . . . . . . . . . . . . . . . . .

4. Temperature Comparisons for a Two-Media RPP with a Sinusoidal Internal Source Using the Adjoint

Formulation . . . . . . . . . . . . . . . 42

5. Temperature Comparisons for a Two-Media RPP with a

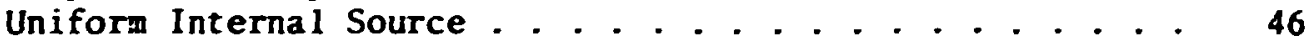

6. Temperature Comparisons for a Two-Media RPP with a Given Surface Temperature Distribution . . . . . . . . . 49

7. Temperature Comparisons for a Three-Media RPP with a Sinusoidal Internal Source . . . . . . . . . . . . 65

8. Temperature Comparisons for a Two-Media RCC with a Functional Internal Source . . . . . . . . . . . 75

9. Temperature Comparisons for DOT Runs with Different Scaling Factors... . . . . . . . . . . . 76

10. Temperature Comparisons for a Two-Media RCC with a Uniform Internal Source ...................

11. Temperature Comparisons for a Two-Media RCC with a Given Surface Temperature Distribution . . . . . . . . 82

12. Temperature Comparisons for a Sphere with a Time Dependent Source . . . . . . . . . . . . . . . 85

13. Temperature Comparisons for a Sphere with a Time Dependent Source and Convective Boundary Condition . . . . 89

14. Temperature Comparisons for Problem Two with the Negat ive Albedo Correction Factor . . . . . . . . . . . . 
15. Temperatures for $\theta=0^{c}$ Compared to Analytical Estimate . . 102

16. Te peratures for $\theta=45^{\circ}$ Compared to Analytical Estimate . - 102

17. Temperatures for $\theta=90^{\circ}$ Corpared to Analytical Estimate . . 103

18. Temperatures for $\theta=135^{\circ}$ Compared to Analytical Estimate . . 103

19. Tesperatures for $\theta=180^{\circ}$ Compared to Analytical Estinate . - 104

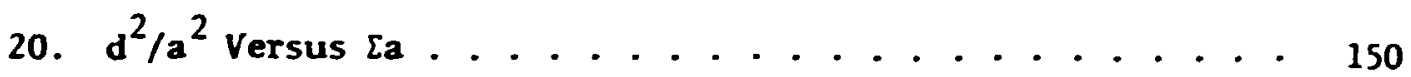




\section{LIST OF FIGURES}

FIQJRE

PAGE

1. Two-Media Sphere with Point Source at Center . . . . . . . 19

2. Two-lledia RPP with Point Source in Medium One . . . . . 24

3. Two-Media RPP with Distributed Source in Medium One . . . 32

4. Three-lfedia RPP with Point Source in Mediur One ...... 50

5. Three-Media RPP with Distributed Source in Medium One . . . 61

6. Two-Media RCC with a Ring Source in Medium One . . . . . 66

7. Two-Media RCC with a Distributed Source in Medium One . . 72

8. Diffusion Theory vs Transport Theory with Different Boundary Conditions ................. 95

9. One-Medium Sphere with Incident Gama Beam . . . . . . . 99

10. Temperatures for $\theta=0^{\circ}$ Compared to Aralytical Estimates................... 105

11. Temperatures for $\theta=45^{\circ}$ Compared to Analytical Estimates.................... 106

12. Temperatures for $\theta=90^{\circ}$ Compared to Analytical Estimates . . . . . . . . . . . . . . . 107

13. Temperatures for $\theta=135^{\circ}$ Compared to Analytical Estimates . . . . . . . . . . . . . . . . 108

14. Temperatures for $\theta=180^{\circ}$ Compared to Analytical Estimates.................. 109 


\begin{abstract}
A new approach in the use of Monte Carlo to solve heat conduction problems was developed using a transport equation approxination to the heat conduction equation. The nethed was shown to be applicable to the solution of multimedia problens in complex geonetries with no inherent Iinitations as to the geonetric complexity of problems which can be solved. Nuclear radiation transport and heat conduction probleas can be calculated with the same computer code; and, in the case whore the nuclear reactions are the heat source, the problem can be performed in a coupled mode with a single computer run.

The method was demonstrated for problems involving multimedia, internal sources, fixed temperature boundary conditions, convective boundary conditions, time-dependent sources, and for a coupled nuclear radiation-heat conduction problem. Comparisons were made to analytical solutions when they were available or could be generated.

The adjoint formulation was found to be the most suitable mode of solution for the class of problems which was considered and therefore the method appears to be more useful for calculating temperatures at specific points rather than temperature distributions.
\end{abstract}


CHAPTER I

INTRODUCTION

Monte Carlo methods are particularly useful in the solution of neutron and gama-ray transport problems which involve complex geometries. Recent work ${ }^{1}$ has suggested that the Monte Carlo approach might also be useful in the solution of certain classes of heat conduction problems.

In this paper a new approach to the use of Monte Carlo in the solution of heat conduction problems is developed. This method, which t.ses a transport equation approximation, will allow the solution of problems with as much geometric complexity as is necessary to adequately describe the physical model. The method is also completely compatible with Monte Carlo radiation transport programs. Thus, criticality, shielding, and heat transfer calculations can be performed with one geometric description and one computer code. Coupled radiation transport and heat conduction problems can also be performed in a single computer run where the heat generated by the nuclear radiation (for exampie, through fission reactions or by energy absorption) is the heat source for the heat transfer calculation.

There are many ways to solve the basic heat conduction problem both numerically and analytically. This method is not intended to replace existing methods. Nurserical techniques such as finite difference or finite element will in general provide a much faster solution. The proposed method will, however, be able to solve certain problems for 
which solutions were not previously feasible. Since the method uses the same logic as existing Monte Carln rudiation transport computer codes, it was not necessary to generate completely new computer programs for its implementation. The solution to the problems solved in this paper were performed using existing computer codes.

Other continuous Monte Carlo techniques for solving heat corduction problems have been reported, ${ }^{1-10}$ and a summary of these methods is given in Reference 1. However, all published results using these methods have been limited to single medium problems. Dissrete Monte Carlo approaches have also been used. ${ }^{11-13}$ These methods are Monte Carlo methods which solve the finite difference equaticiis and have not proven to be competitive with other finite difference techniques. Hybrid Mnnte Carlo approaches have also been developed, ${ }^{14,15}$ but these require the use of both analog and digital systems and appear to be limited in their potential applications.

The equations of heat conduction and their relationship to the transport equation approximation in its solution are described in Chapter II. Chapter III provides a description of the application of Monte Carlo to the solution of the transport equation.

The validity of the method is established in Chapter IV where Monte Carlo solutions are compared to analytical results for a wide range of problems. Since a major area of interest is the demonstration of the applicability of the transport equation approximation to multimedia problems, and analytical solutions were available for only a very limited number of multimedia problems, Chapter IV also includes 
the development of the analytical solutions for those problems for which solutions were not previously available. The verification of these solutions is given in Appendix A. A derivation of a bounded next event (flux at a point) estimator which was used in a Monte Carlo solution in Chapter IV is given in Appendix B.

Methods is correct for the differences between transport theory and diffusion theory as applied to heat conduction are developed in Chapter V.

A coupled gamma-ray, heat conduction problem is solved ill Chapter VI as an example of the type of problem for which this method is applicable. A listing of the input data and output for this problem is given in Appendix $C$.

Coriclusions and recommendations are given in Chapter VII. 
CHAPTER II

TRANSPORT THEORY APPROXIMATION TO THE DIFFERENTIAL EQUATION OF HEAT CONDUCTION

The differential equation of heat conduction through a stationary, isotropic solid is given by: 16

$$
\nabla \cdot K(\bar{r}, T) \nabla T(\bar{r}, t)+S(r, t)=\rho c \frac{\partial T}{\partial t},
$$

where

$$
\begin{aligned}
K(\bar{r}, T) & =\text { thermal conductivity } \\
\rho & =\text { density } \\
c & =\text { specific heat } \\
S(\bar{r}, t) & =\text { internal heat source }
\end{aligned}
$$

If the thermal conductivity is not a function of position or temperature, this simplifies to:

$$
K \nabla^{2} T(\bar{r}, t)+S(\bar{r}, t)=\rho c \frac{\partial T}{\partial t}
$$

It is desired to solve the above differential equation subject to the following types of standard boundary conditions.

1. Boundary condition of the first kind:

The temperature is specified along a boundary surface.

$$
T=f\left(\vec{r}_{s}, t\right)
$$

where $\quad \bar{r}_{s}$ is on a boundary surface. 
2. Boundary condition ot the second kind:

The normal derivative of the tempizature is specified along a boundary surface.

$$
\frac{\partial T}{\partial n}=f\left(\bar{r}_{s}, t\right)
$$

where $\quad \dot{\mathbf{r}}_{s}$ is on a boundary surface, and $\partial / \partial n$ represents differentiation along the outward normal to the surface.

3. Boundary condition of the third kind:

A combination of the temperature and normal derivative is specified at a boundary.

$$
K \frac{\partial T}{\partial \mathbf{n}}+h T=f\left(\bar{r}_{S}, t\right)
$$

where $\quad \dot{r}_{s}$ is on a boundary surface, $\partial / \partial n$ represents differentiation along the outward normal to the surface, $K$ is the thermal conductivity, and $h$ is the consective heat transfer coefficient. This type of boundary condition is normally associated with forced convection and written as

$$
K \frac{\partial T}{\partial n}=h\left(T_{\infty}-T\right)
$$

where $T_{\infty}$ is the temperature of the surroundings.

4. Natural convection boundary condition:

$$
K \frac{\partial T}{\partial n}=h\left(T_{\infty}-T\right)^{5 / 4}
$$


5. Thernal radiation boundary condition:

$$
K \frac{\partial T}{\partial n}=\sigma \varepsilon\left(T_{\infty}^{4}-T^{4}\right)
$$

where $\sigma$ is the Stefan-Boltzmann constant

$E$ is the enissivity of the surface.

Across interfaces between two different media

$$
K_{1} \frac{\partial T_{1}}{\partial n}=K_{2} \frac{\partial T_{2}}{\partial n}
$$

where $K_{1}$ and $T_{1}$ are near the boundary in the first medium and $K_{2}$ and $T_{2}$ are near the boundary in the second medium and $\mathrm{T}_{1}=\mathrm{T}_{2}$ at boundary.

The neutron diffusior. equation can be written as 17

$$
D \nabla^{2} \phi(\bar{r}, t)-\Sigma_{a} \phi(\bar{r}, t)+S(\bar{r}, t)=\frac{1}{V} \frac{\partial \phi}{\partial t},
$$

where $\phi$ is the neutron flux

D is the diffusion coefficient.

$\Sigma_{a}$ is the absorption cross section, and

$y$ is the neutron's speed.

D is normally given by

$$
D=\frac{1}{3\left(\Sigma-\mu \Sigma_{5}\right)},
$$

where $\Sigma$ is the total cross section,

$\bar{\mu}$ is the average cosine of the scattering angle, and

$\Sigma_{s}$ is the scattering cross section. 
If $\Sigma_{a}=0$, then $\Sigma=\Sigma_{s}$ and

$$
D=\frac{1}{3 \Sigma(1-\bar{\mu})}
$$

If the scattering is isotropic, $\bar{\mu}=0$, and

$$
\mathrm{D}=\frac{1}{3 \Sigma}
$$

Therefore, under these condition, the diffusion equation becomes

$$
\frac{1}{3 \Sigma} \nabla^{2} \phi+S(\bar{r}, t)=\frac{1}{v} \frac{\partial \phi}{\partial t},
$$

and by comparing the above to the heat conduction equation, the two equations are equivalent if

$$
\Sigma=\frac{1}{3 K},
$$

and

$$
v=\frac{1}{\rho c} \text {. }
$$

In neutron transport theory problems, the diffusion equation is frequently used as an approximation to the Boltzmann transport equation. Monte Carlo methods are available for solving the transport equation in problems with very complex geometries and these methods may then be used to approximate the solutions to the equivalent heat conduction problems.

Transport theory differs from diffusion theory when scattering is highly anisotropic or the angular distribution of particles (angular 
flux) is highly anisotropic. Transport theory also differs from diffusion theory in the application of various boundary conditions. For example, diffusion theory allows the specification of a zero f'ux boundary condition shereas transport theory allows only a zero incoming flux boundary convition.

In trying to adjust neutron diffusion theory to approxinate the transport equation, various methods of correction have been developed. For example, to account for anisotropic scattering the diffusion coefficient is modified by the average scattering angle and a zero flux boundary condition at ars extrapolated distance ${ }^{18}$ from the boundary is used to simlate a zerc incoming flux at the boundary. In optically thin regions (very few mean free paths thick), in the vicinity of strong absorbers or sources o when scattering is strongly anisotropic, the differences between the neutron diffusion approximation and transport theory may be significant and in these cases, the use of neutron diffusion theory is inappropriate. These differences are more easily corrected when transport theory is used to approximate diffusion theory. Diffusion theory is based $r n$ isotropic scattering and the scattering used in the transport solution can always be made isotropic. To correct for the anisotropic flux which transport theory will give in the vicinity of strong absorbers or sources or in optically thin regions, the actual cross sections used in ihe transport calculation can be scaled so that the regions of interest are no longer optically thin. An anisotropic flux will therefore exist only over a small region of the 
problem and the transport theory approxination to the diffusion equation will be appropriate.

Scaling of cross sections is accomplished by letting $T=B \phi$ where $B$ is a constant.

Then

$$
K B \nabla^{2} \phi+S(\bar{r}, t)=B \rho C \frac{\partial \phi}{\partial t}
$$

1. Boundary condition of first kind:

$$
\phi=\frac{f\left(r_{S}, t\right)}{B} .
$$

2. Boundary condition of second $k$ ind:

$$
\frac{\partial \phi}{\partial n}=\frac{f\left(r_{s}, t\right)}{B} .
$$

3. Boundary condition of third kind:

$$
K E \frac{\partial \phi}{\partial n}+n B \phi=f\left(r_{s}, t\right) .
$$

4. Natural convection boundary condition:

$$
K B \frac{\partial \phi}{\partial n}=h \beta^{5 / 4}\left(\phi_{\infty}-\Phi\right)^{5 / 4} \text {. }
$$

5. Thermal radiation boundary condition:

$$
K B \frac{\partial \phi}{\partial n}=\sigma \in \beta^{4}\left(\phi_{\infty}^{4}-\phi^{4}\right)
$$


across interface

$$
K_{1} \beta \frac{\partial \phi_{1}}{\partial n}=K_{2} \beta \frac{\partial \phi_{2}}{\partial n}
$$

and

$$
B \phi_{1}=B \phi_{2}
$$

Therefore, by letting $T=B \phi$ and comparing to the diffusion equation

$$
\Sigma=\frac{1}{3 K B},
$$

and

$$
v=\frac{1}{\rho c \beta}
$$

The source duesn't change, $S=S(\bar{r}, t)$ and the above revised boundary conditions should be used.

The method of solution using the transport equation approximation is then tc scive the Boltzmann transport equation for the flux $\phi$ under conditions of isotropic scattering and no absorption. The actual cross sections and velocities used in the calculation may be scaled using Equations $(2-25)$ and $(2-26)$ so as to make the differences between the transport equation solution and the difrusion equation solution as small as desired (with a penalty of increasing computation time). The boundary conditions used during the calculation are obtained from Equations (2-18) through (2-24). Since the diffusion equation is equivalent to the heat conduction equation under the above conditions, 
11

the transport equation approximation of the solution to the heat condition equation is then obtained from

$$
T=B
$$

The next chapter describes the application of Monte Carlo to the solution of the Boltzmann transport equation. 


\section{CHAPTER III}

\section{APPLICATION OF MONTE CARLO TO THE TRANSPORT EQUATION}

As shown in the previous chapter, the solution of a heat conduction proble can be approxinated by the solution of an appropriate transport proble. Once the appropriate transport problem has been defined, any of the methods which would normally be used in its solution can be used without additional justification. Monte Carlo ethods have been developed for solving transport problems and the previously developed nethodology is thereby appropriate. A mathematical treatnent of the application of the Monte Carlo method to solve the Boltzaann transport equation is given in a book by Spanier and Gelbard, 19 and a review of the Monte Carlo method as applied to particle transport is given by Carter and Cashwel1. 20

Only a brief discussion of the application of Monte Carlo methods to the solution of the transport equation will be given here. The formulation given is based primarily on the article by Coveyou, Cain, and Yost. 21

The integral form of the transport equation can be written as

$$
\psi(x)=S(x)+\int_{\Omega} \psi(y) G(y, x) d y
$$

where $\psi(x)$ is the event density (collisions and leakage are considered events)

$S(x)$ is the first event source distribution function $G(y, x)$ is the transition kernel (the probability of an event at $x$ given an event at $y$ ), and 
$x, y$ are vectors in the space $\Omega$ of interest and may include position, direction, time, and energy dependence.

$\int G(x, y) d y$ represents the nonabsorption probability at $x$. $\Omega$

The flux at any point in a mediua is obtained from the event density by dividing by the cross section of the mediun.

For nonwultiplying media

$$
\int_{\Omega} G(x, y) d y \leq 1 \text { for all } x \in \Omega .
$$

For the case of heat transfer all media will be nonabsorbing and

$$
\int_{\Omega} G(x, y) d y=\left\{\begin{array}{l}
1 \text { for all } x \text { at collision sites, } \\
0 \text { for all } x \text { at leakage sites. }
\end{array}\right.
$$

The usual problem is to calculate an effect of interest $\lambda$, which is given by the functional

$$
\lambda=\int_{\Omega} \psi(x) R(x) d x
$$

where $R(x)$ is the response function.

The adjoint formulation of the same problem can be written

$$
\tilde{\Psi}(x)=R(x)+\int_{\Omega} G(x, y) \tilde{\Psi}(y) d y,
$$

and

$$
\lambda=\int_{\Omega} \tilde{\psi}(x) S(x) d x .
$$

Note that in the adjoint formulation the integration of the transition kernel is with respect to the second variable instead of the first. The integral equation for $\psi$ will be referred to as the forward formulation to differentiate it from the adjoint formulation. 
Let

$$
\psi(x)=\sum_{p=0}^{q} \phi_{p}(x)
$$

where

$$
\psi_{0}(x)=S(x)
$$

and

$$
\psi_{p+1}(x)=\int_{\Omega} \psi_{p}(y) G(y, x) d y
$$

Then

$$
\int_{\Omega} \psi(y) G(y, x) d y=\int_{\Omega_{p}=0}^{q} \psi_{p}(y) G(y, x) d y
$$

The sumation, if it exists (existence is discussed in Chapter Three of Reference 19) is absolutely convergent since $\psi_{p}(y) \geq 0$ and $G(y, x) \geq 0$ and therefore sumation and integration can be interchanged giving

$$
\int_{\Omega} \Psi(y) G(y, x) d y=\sum_{p=0} \int_{\Omega} \psi_{p}(y) G(y, x) d y=\sum_{p=0}^{q} \psi_{p+1}(x)=\sum_{p=1}^{\infty} \Psi_{p}(x) .
$$

Therefore, adding $S(x)$ (or $\Psi_{0}(x)$ ) to both sides gives

$$
S(x)+\int_{\Omega}(y)^{r}(y, x) d y=\psi(x)
$$

and $\Psi(x)$ as defined by Equation (3-7) satisfies the integral form of the transport equation. 
Rewriting gives:

$$
\begin{aligned}
\psi\left(x_{1}\right)=S\left(x_{1}\right)+ & \int_{\Omega} S\left(x_{2}\right) G\left(x_{2}, x_{1} j d x_{2}\right. \\
& +\int_{\Omega} G\left(x_{3}, x_{1}\right) \int_{\Omega} S\left(x_{2}\right) G\left(x_{2}, x_{3}\right) d x_{2} d x_{3} \\
& +\int_{\Omega} G\left(x_{4}, x_{1}\right) \int_{\Omega} G\left(x_{3}, x_{4}\right) \int_{\Omega} S\left(x_{2}\right) G\left(x_{2}, x_{3}\right) d x_{2} d x_{3} d x_{4} \\
& +\ldots
\end{aligned}
$$

And $\lambda$ is given by

$$
\lambda=\int_{\Omega} \psi\left(\mathrm{x}_{1}\right) \mathrm{R}\left(\mathrm{x}_{1}\right) \mathrm{d} \mathrm{x}_{1}
$$

or

$$
\begin{aligned}
\lambda= & \int_{\Omega} S\left(x_{1}\right) R\left(x_{1}\right) d x_{1}+\int_{\Omega} \int R\left(x_{1}\right) S\left(x_{2}\right) G\left(x_{2}, x_{1}\right) d x_{2} d x_{1} \\
& +\int_{\Omega} \int R\left(x_{1}\right) G\left(x_{3}, x_{1}\right) \int_{\Omega} S\left(x_{2} j G\left(x_{2}, x_{3}\right) d x_{2} d x_{3} d x_{1}\right. \\
& +\int_{\Omega} \int R\left(x_{1}\right) G\left(x_{4}, x_{1}\right) \int_{\Omega} G\left(x_{3}, x_{4}\right) \int_{\Omega} S\left(x_{2}\right) G\left(x_{2}, x_{3}\right) d x_{2} d x_{3} d x_{4} d x_{1} \\
& +\ldots . .
\end{aligned}
$$

0r, interchanging dumy integration variables,

$$
\begin{aligned}
\lambda= & \int_{\Omega} S\left(x_{1}\right) R\left(x_{1}\right) d x_{1}+\iint_{\Omega} R\left(x_{2}\right) S\left(x_{1}\right) G\left(x_{1}, x_{2}\right) d x_{1} d x_{2} \\
& +\int_{\Omega \Omega} \int R\left(x_{3}\right) G\left(x_{2}, x_{3}\right) \underset{\Omega}{\int S\left(x_{1}\right) G\left(x_{1}, x_{2}\right) d x_{1} d x_{2} d x_{3}} \\
& +\int_{\Omega \Omega} \int R\left(x_{4}\right) G\left(x_{3}, x_{4}\right) \int_{\Omega} G\left(x_{2}, x_{3}\right) \int_{\Omega} S\left(x_{1}\right) G\left(x_{1}, x_{2}\right) d x_{1} d x_{2} d x_{3} d x_{4}
\end{aligned}
$$$$
+. \cdot .
$$ 
The above formulation ma be used to construct a Monte Carlo randon walk. 1) A position $x_{1}$ is picked fron $S(x)$ and $R\left(x_{1}\right)$ is scored. This is an estimate of the first integral. 2) Starting at $x_{1}$, a new position $x_{2}$ is picked using $G\left(x_{1}, y\right)$ and a value $w_{1}$ is assigned by

$$
w_{1}=\int_{S_{6}} G\left(x_{1}, y\right) d y
$$

The product $w_{1} \cdot R\left(x_{2}\right)$ is then scored as the estimate of the second integral. 3) Starting at $x_{2}$ a new position $x_{3}$ is picked using $G\left(x_{2}, v\right)$ and a value $w_{2}$ is assigned by

$$
w_{2}=w_{1} \int_{\Omega} G\left(x_{2}, y\right) d y .
$$

The product $w_{2} \cdot R\left(x_{3}\right)$ is then scored as the estimate of the third integral, 4) ....

This process is continued until an external boundary is reached or until the value of $w$ is so small that additional scores of $w \cdot R(x)$ would be negligible. In this case Russian roulette may be used to determine if the random walk is to be continued.

The random walk which comprises the series of points $x_{1}, x_{2}, \ldots$ in $\Omega$ will be called a history.

The cumulative score from the history is then an estimate of $\lambda$. If a large number of histories are performed, the mean will be a reasonable estimate of $\lambda$. The variance of the estimations should also be computed to provide an indication of the statistical uncertainty of the estimation. 
Similarly, the adjoint formulation of the solution for $\lambda$ can be written

$$
\lambda=\int_{\Omega} R\left(x_{1}\right) S\left(x_{1}\right) \mathrm{d} x_{1}+\iint_{\Omega} S\left(x_{2}\right) R\left(x_{1}\right) G\left(x_{2}, x_{1}\right) d x_{1} d x_{2}+\ldots
$$

Note that this is identical to the forward formulation with the order of integration interchanged.

The Monte Carlo solution of the adjoint formulation is accomplished in a similar manner except that $x_{1}$ is chosen from $R(x)$ and $x_{2}$ is chosen from $G\left(x, x_{1}\right)$, i.e., given a collision at $x_{1}, G\left(x, x_{1}\right)$ is the probability that it was preceded by a collision at $x$.

A number $\because$ techniques have been developed to reduce the variance of the estimate of $\lambda$ for given computational times. Some of the more common techniques include source biasing, Russian roulette, splitting, and exponential transform (path length stretching). Standard techniques are also available for obtaining an estimate of $\lambda$ from the Monte Carlo histories. Although only a few of these techniques are used in the following sections in the solution of the demonstration problems, all of the techniques which have been deseloped for the Monte Carlo solution of the transport equation are available for use. 
CHAPTER IV

\section{COMPARISONS OF MONIE CARLO TRANSPORT SOLUTIONS}

TO ANALYTICAL SOLUTIONS

In this chapter Monte Carlo solutions are formulated for a number of multimedia problems and compared against analytical solutions. Since analytical solutions were not available in the literature for multimedja problems involving more than rne dimension, ${ }^{22}$ the development of the analytical solutions for these problems is also presented.

\section{TWO MEDIA SPHERE}

Problem 1: To calculate the temperature at several points within a one-dimensional, two-media sphere (F1gure 1). Assume:

1. A point source of strength $s$ at the center

2. $K_{1}$ is the thermal corductivity for $0 \leq r \leq a$

3. $k_{2}$ is the thermal conductivity for $a \leq r \leq b$

a. No contact resistance between media

5. $T(r=b)=80^{\circ} \mathrm{C}$

6. steady state.

The analytical sulution can be obtained from Carslaw and Jaeger. 22

$$
\begin{array}{ll}
T(r)=\frac{s}{4 \pi K_{1} T}+\frac{s\left[b\left(K_{1}-K_{2}\right)-a K_{1}\right]}{4 \pi K_{1} K_{2} a b}+T_{b}, 0 \leq r \leq a . \\
T(r)=\frac{s}{4 \pi K_{2} r}-\frac{s}{4 \pi K_{2} b}+T_{b}, & a \leq r \leq b .
\end{array}
$$




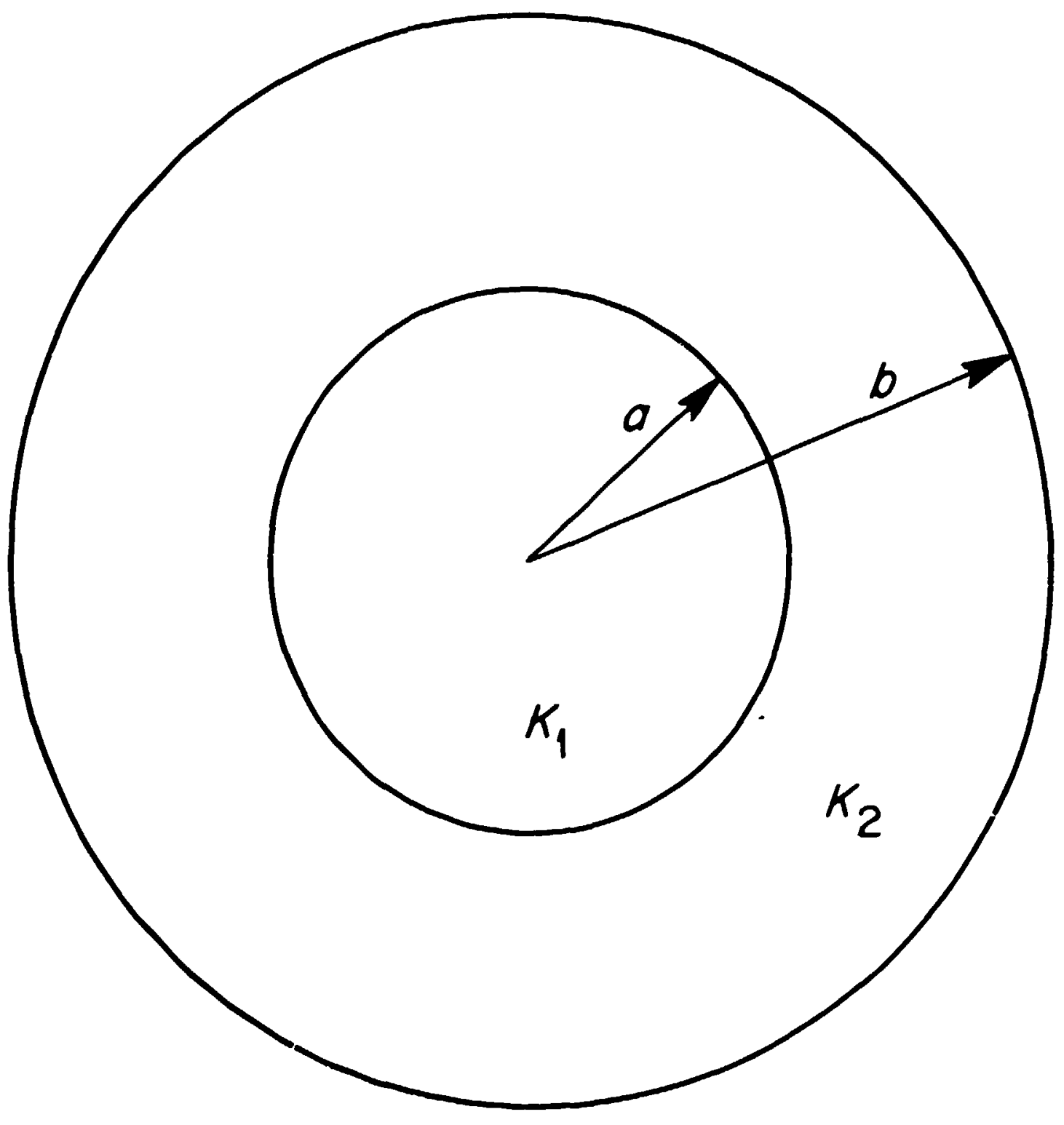

Figure 1. Two-media sphere with point source at center. 
The paraneters for the probien are:

$$
\begin{aligned}
& K_{1}=.3333 \mathrm{cal} / \mathrm{sec} \mathrm{cm}^{\circ} \mathrm{C} \\
& K_{2}=.1667 \mathrm{cal} / \mathrm{sec} \mathrm{cm}^{\circ} \mathrm{C} \\
& a=5 \mathrm{~cm} \\
& b=10 \mathrm{~cm} \\
& s=238.8 \mathrm{cal} / \mathrm{sec}(1000 \text { watts). }
\end{aligned}
$$

This gives:

$$
T(r)=\left\{\begin{array}{cl}
\frac{57.0}{r}+80.0 & 0 \leq r \leq 5 \\
\frac{114.0}{r}+68.6 & 5 \leq r \leq 10
\end{array},\right.
$$

where the temperature is given in ${ }^{\circ} \mathrm{C}$.

The Monte Carlo calculations were performed using the MORSE-SGC Morite Carlo neutron and gamma-ray transport code, ${ }^{23}$ which provides a Monte Carlo solution to either the forward or adjoint Boltzmann transport equation.

The parameters used in the transport equation solution (using the conversion in Chapter II) were
1. $\Sigma_{1}=\frac{1}{3 \mathrm{~K}_{1}}=1 \mathrm{~cm}^{-1}$
2. $\Sigma_{2}=\frac{1}{3 F_{2}}=2 \mathrm{~cm}^{-1}$
3. $a=5 \mathrm{~cm}$
4. $b=10 \mathrm{~cm}$
5. $\mathrm{s}=238.8 / \mathrm{sec}$ 
and the flux $\phi$ has units of $\mathrm{cm}^{-2} \mathrm{sec}^{-1}$. The flux was calculated at $1 \mathrm{~cm}$ radial intervals in the sphere using a boundary crossing estimator. The solution was obtained using the forward formulation. The results of these calculations are given in Table 1 along with the analytical results. (The estimate of the standard deviation of the Monte Carlo solution is also given). The Monte Carlo results were obtained using 4.5 min of CPU time on the IBM $369 / 91$.

To more accurately approximate the diffusion equation by the transport equation, an extrapolation distance correction could have been made to account for edge effects. The extrapolation distance $d$ is given by:

$$
\mathrm{d}=\frac{.71}{\Sigma_{2}}=.355 \mathrm{~cm} .
$$

This distance would be subtracted from the radius giving an outer radius of $19.645 \mathrm{~cm}$. The Monte Carlo solution as performed corresponds to the solution of a problem with outer radius of $20.355 \mathrm{~cm}$, if the extrapolation distance correction is incorporated.

The analytical solution for a composite sphere with an outer radius of $20.355 \mathrm{~cm}$ is included in the table to show the effects of including the correction. The Monte Carlo solution to the transport equation compares very well to the analytical solution once this correction is made, except in the vicinity of the point source. 
TABLE 1

TEMPERATURE COMPARISONS FOR A TWO-MEDIA SPHERE WITH A POINT SOURCE

\begin{tabular}{ccccc}
\hline $\begin{array}{c}\text { Radius } \\
\text { (cm) }\end{array}$ & $\begin{array}{c}\text { Analytic } \\
\text { Temperature }\end{array}$ & $\begin{array}{c}\text { Analytic Temperature } \\
\text { with Extrapolation } \\
\text { Distance Correction }\end{array}$ & $\begin{array}{c}\text { Monte Carlo } \\
\text { Temperature } \\
{ }^{\circ} \mathrm{C}\end{array}$ & $\begin{array}{c}\text { Estimated } \\
\text { Standard } \\
\text { Deviation } \\
\mathrm{C}^{\circ}\end{array}$ \\
\hline 1.0 & 137.0 & 137.4 & 142 & 1.8 \\
2.0 & 108.5 & 108.9 & 108 & 0.70 \\
3.0 & 99.00 & 99.39 & 98.6 & 0.54 \\
4.0 & 94.25 & 94.64 & 93.6 & 0.44 \\
5.0 & 91.40 & 91.79 & 91.2 & 0.26 \\
6.0 & 87.6 & 87.99 & 87.8 & 0.17 \\
7.0 & 84.89 & 85.28 & 85.2 & 0.14 \\
8.0 & 82.85 & 83.24 & 83.2 & 0.083 \\
9.0 & 81.27 & 81.66 & 81.7 & 0.047 \\
10.0 & 80.00 & 80.39 & 80.3 & 0.0004 \\
\hline
\end{tabular}

Note: 11 numbers calculated with Monte Carlo in this and subsequent tables will be given with an estimation of the standard deviation of the numbers. 


\section{TWO MEDIA RPP GREEN'S FUNCTION}

The next series of problems requires analytical solutions for threeiimensional, two-media rectangular parallelepipeds. The first step in generating the solutions to these problems is to derive an appropriate Green's function (Figure 2).

From Carslaw and Jaeger, ${ }^{22}$ the one-medium Green's function for a rectangular parallelepiped (RPP) $0 \leq x \leq a, 0 \leq y \leq b, 0 \leq z \leq c$, with zero surface temperature and a point source at $\left(x^{\prime}, y^{\prime}, z^{\prime}, \tau\right)$ is

$$
\begin{aligned}
T= & \frac{8}{a b c} \sum_{\ell=1 m=1 n=1}^{\infty} \sum_{l=1}^{\infty}\left[\sin \frac{\ell \pi x}{a} \sin \frac{\ell \pi x^{\prime}}{a} \sin \frac{m \pi y}{b} \sin \frac{m \pi y^{\prime}}{b} \sin \frac{n \pi z}{c} \sin \frac{n \pi z^{\prime}}{c}\right. \\
& e^{\left.-K \pi^{2}(t-\tau)\left(\frac{l^{2}}{a^{2}}+\frac{m^{2}}{b^{2}}+\frac{n^{2}}{c^{2}}\right)\right] .}
\end{aligned}
$$

To obtain the time independent Green's function integrate $\int_{0}^{t} d \tau$ and take $\lim t \rightarrow \infty$, to get

$$
T=\frac{8}{a b c k \pi^{2}} \sum_{l=1 m=1 n=1}^{\infty} \sum_{\sum}^{\infty} \frac{1}{\frac{l^{2}}{a^{2}}+\frac{m^{2}}{b^{2}}+\frac{n^{2}}{c^{2}}} \sin \frac{l \pi x}{a} \sin \frac{l \pi x^{\prime}}{a} \sin \frac{m \pi y}{b} \sin \frac{m \pi y^{\prime}}{b} \sin \frac{n \pi z}{c} \sin \frac{n \pi z^{\prime}}{c} .
$$

Also from Carslaw and Jaeger, ${ }^{22}$ if the RPP face at $x=0$ is maintained at $\phi(y, z, t)$, other faces at zero, with initial conditions $T(x, y, z, 0)=0$

$$
\begin{gathered}
T=\frac{8 K \pi}{a^{2} b c} \sum_{l=1 m=1 n=1}^{\infty} \sum_{l=1}^{q}\left[\ell \sin \frac{l \pi x}{a} \sin \frac{m \pi y}{b} \sin \frac{n \pi z}{c} \int_{0}^{t} d \tau \int_{0}^{c b} \int_{0}^{b} \sin \frac{m \pi y^{\prime}}{b} \sin \frac{n \pi z^{\prime}}{c} \phi\left(y^{\prime}, z^{\prime}, \tau\right)\right. \\
\cdot e^{\left.-K \pi^{2}(t-\tau)\left(\frac{l^{2}}{a^{2}}+\frac{m^{2}}{b^{2}}+\frac{n^{2}}{c^{2}}\right) d y^{\prime} d z^{\prime}\right]}
\end{gathered}
$$




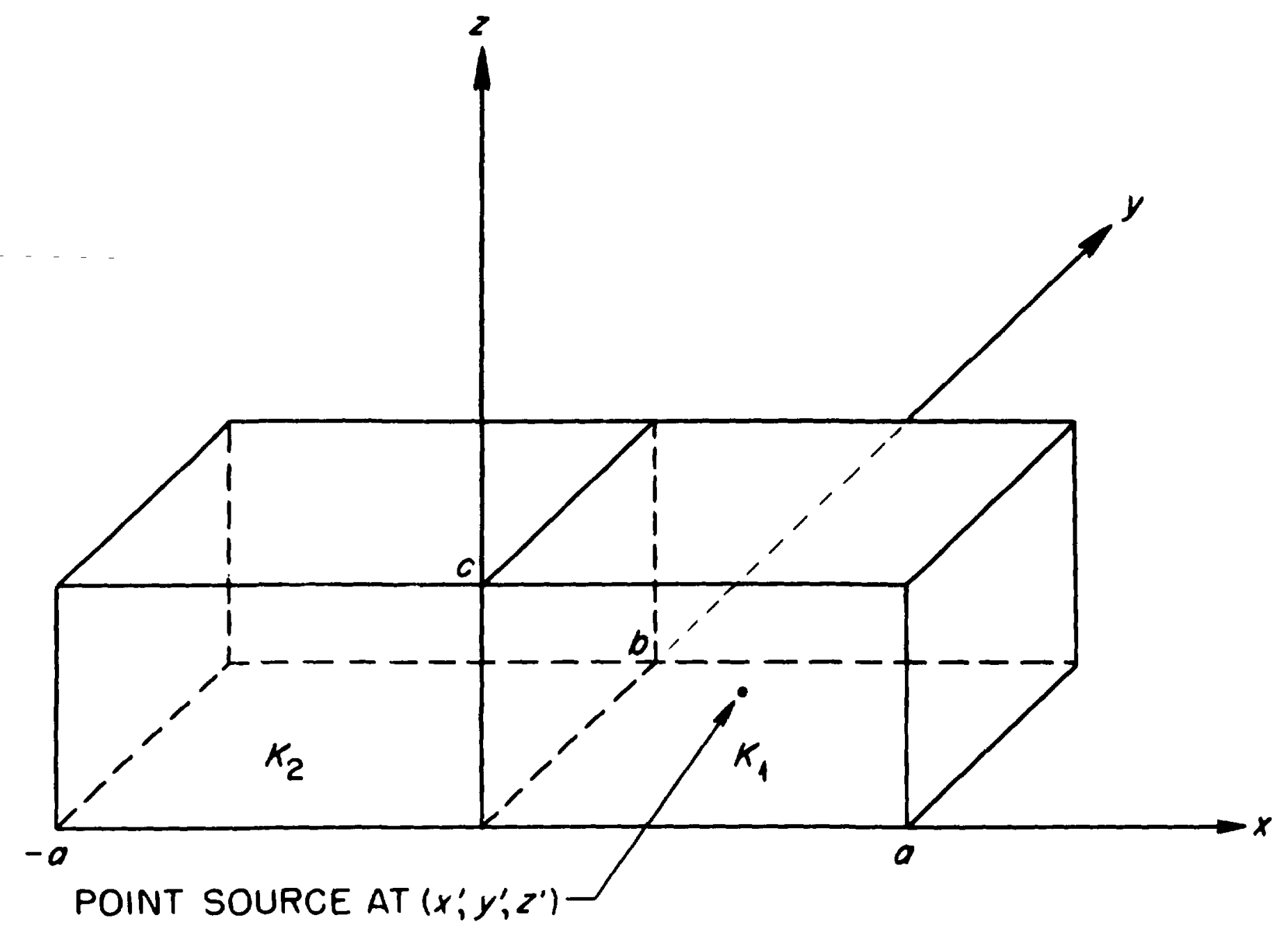

Figure 2. Two-media RPP with point source in modium one. 
Let $\phi(y, z, t)=\phi(y, z)$ and take $\lim t \rightarrow \infty$ to get the steady-state solution

$$
\begin{aligned}
& T=\frac{4}{b c} \sum_{\ell=1 m=1 n=1}^{q} \sum_{l}^{q} \mid \frac{2 l \pi}{\ell^{2} \pi^{2}+a^{2} \pi^{2}\left(\frac{m^{2}}{b^{2}}+\frac{n^{2}}{c^{2}}\right)^{\sin \frac{l \pi x}{a}} \sin \frac{m \pi y}{b} \sin \frac{n \pi z}{c}} \\
& \left.\int_{0}^{c} \int_{0}^{b} \phi\left(y^{\prime}, z^{\prime}\right) \sin \frac{m \pi y^{\prime}}{b} \sin \frac{n \pi z^{\prime}}{c} d y^{\prime} d z^{\prime}\right|^{\prime}
\end{aligned}
$$

But

$$
\sum_{l=1}^{\infty} \frac{2 \ell \pi}{l^{2} \pi^{2}+a^{2} \pi^{2}\left(\frac{m^{2}}{b^{2}}+\frac{n^{2}}{c^{2}}\right)} \sin \frac{\ell \pi x}{a}=\frac{\sinh \left[\pi(a-x)\left(\frac{m^{2}}{b^{2}}+\frac{n^{2}}{c^{2}}\right)^{1 / 2}\right]}{\sinh \left[\pi a\left(\frac{m^{2}}{b^{2}}+\frac{n^{2}}{c^{2}}\right)^{1 / 2}\right]} .
$$

Thrrefore

$$
T=\frac{4}{b c}{\underset{\sum}{m=1 n=1} \infty}_{\sum}^{\infty}\left\{\frac{\sinh \left[\pi(a-x)\left(\frac{m^{2}}{b^{2}}+\frac{n^{2}}{c^{2}}\right)^{1 / 2}\right.}{\sinh [\pi a} \frac{\left[\frac{m^{2}}{b^{2}}+\frac{n^{2}}{c^{2}}\right)^{1 / 2}}{\sin \frac{m \pi y}{b} \sin \frac{n \pi z}{c}}\right.
$$

To obtain a Green's function for a composite, two-media region, first assume an RPP $-a \leq x \leq 0,0 \leq y \leq b, 0 \leq z \leq c, K=K_{2}, \phi(y, z)$ at $x=0$, other faces at ze: 0 , then 


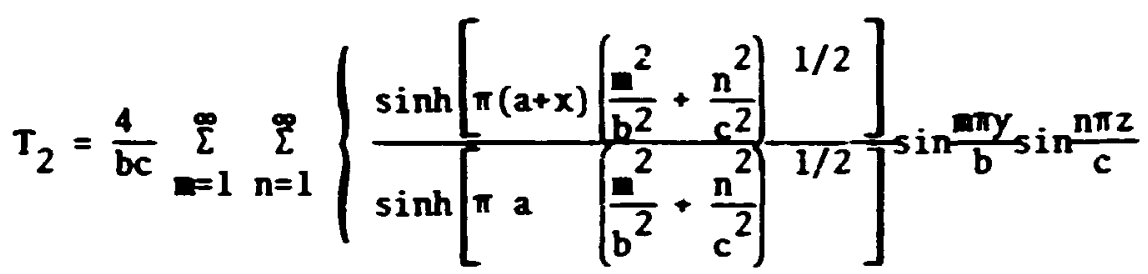

$$
\begin{aligned}
& \int_{0}^{c} \int_{0}^{b} \phi\left(y^{\prime}, z^{\prime}\right) \sin \frac{n \pi y^{\prime}}{b} \sin \frac{n \pi z^{\prime}}{c} d y^{\prime} d z^{\prime} \mid
\end{aligned}
$$

Now assume RPP $0 \leq x \leq z, 0 \leq y \leq b, 0 \leq z \leq c, K=K_{1}, \phi(y, z)$ at $x=0$, other faces zero, and point source at $\left(x^{\prime}, y^{\prime}, z^{\prime}\right)$, then

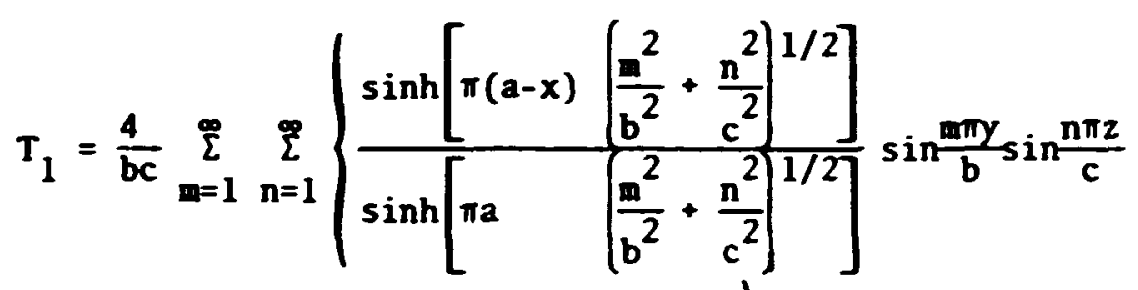

$$
\begin{aligned}
& \left.\cdot \int_{0}^{c} \int_{0}^{b} \phi\left(y^{\prime}, z^{\prime}\right) \sin \frac{m \pi y^{\prime}}{b} \sin \frac{n \pi z^{\prime}}{c} d y^{\prime} d z^{\prime}\right|_{l=1 m=1 n=1} ^{\infty} \mid \frac{8}{k_{1} \pi^{2} a b c}
\end{aligned}
$$

For continuity of heat flow at $x=0$

$$
\left.K_{2} \frac{\partial T_{2}}{\partial x}\right|_{x=0}=\left.K_{1} \frac{\partial T_{1}}{\partial x}\right|_{x=0}
$$


From Equation (4-11)

$$
\begin{aligned}
& \left.\frac{\partial T_{2}}{\partial x}\right|_{x=0}=\frac{4 \pi}{b c} \sum_{n=1}^{\infty} \sum_{n=1}^{\infty}\left\{\left(\frac{m^{2}}{b^{2}}+\frac{n^{2}}{c^{2}}\right)^{1 / 2} \frac{\cosh \pi a\left(\frac{m^{2}}{b^{2}}+\frac{n^{2}}{c^{2}}\right)^{1 / 2}}{\sinh \pi a\left(\frac{m^{2}}{b^{2}}+\frac{n^{2}}{c^{2}}\right)^{1 / 2} \sin \frac{m \pi y}{b} \sin \frac{n \pi z}{c}}\right. \\
& \left.\cdot \int_{0}^{c} \int_{0}^{b} \phi\left(y^{\prime}, z^{\prime}\right) \sin \frac{m \pi y^{\prime}}{b} \sin \frac{n \pi z^{\prime}}{c} d y^{\prime} d z^{\prime}\right\} \text {. }
\end{aligned}
$$

From Equation (4-12)

$$
\begin{aligned}
& \left.\frac{\partial T}{\partial x}\right|_{x=0}=-\frac{4 \pi}{b c} \sum_{m=1}^{\infty} \sum_{n=1}^{\infty}\left\{\left(\frac{m^{2}}{b^{2}}+\frac{n^{2}}{c^{2}}\right)^{1 / 2} \frac{\cosh \pi a\left(\frac{m^{2}}{b^{2}}+\frac{n^{2}}{c^{2}}\right)^{1 / 2}}{\sinh \pi a\left(\frac{m^{2}}{b^{2}}+\frac{n^{2}}{c^{2}}\right)^{1 . / 2}} \sin \frac{m \pi z}{b} \sin \frac{n \pi z}{c}\right. \\
& \left.\cdot \int_{0}^{c} \int_{0}^{b} \phi\left(y^{\prime}, z^{\prime}\right) \sin \frac{m \pi y^{\prime}}{b} \sin \frac{n \pi z^{\prime}}{c} d y^{\prime} d z^{\prime}\right\}+\frac{4}{k_{1} b c} \\
& -\sum_{\ell=1}^{\infty} \sum_{m=1}^{\infty} \sum_{n=1}^{\infty} \frac{2 l \pi}{l^{2} \pi^{2}+a^{2} \pi^{2}\left(\frac{m^{2}}{b^{2}}+\frac{n^{2}}{c^{2}}\right)} \sin \frac{l \pi x^{\prime}}{a} \sin \frac{m \pi y}{b} \sin \frac{m \pi y^{\prime}}{b} \sin \frac{n \pi z}{c} \sin \frac{n \pi z^{\prime}}{c} .
\end{aligned}
$$

Using

$$
\sum_{l=1}^{\infty} \frac{2 \ell \pi}{l^{2} \pi^{2}+a^{2} \pi^{2}\left(\frac{m^{2}}{b^{2}}+\frac{n^{2}}{c^{2}}\right)^{\sin \frac{l \pi x^{\prime}}{a}}}=\frac{\sinh \left[\pi\left(a-x^{\prime}\right)\left(\frac{m^{2}}{b^{2}}+\frac{n^{2}}{c^{2}}\right)^{1 / 2}\right]}{\sinh \left[\pi a\left(\frac{m^{2}}{b^{2}}+\frac{n^{2}}{c^{2}}\right)^{1 / 2}\right]},
$$


and setting

$$
\left.K_{2} \frac{\partial T_{2}}{\partial x}\right|_{x=0}=\left.K_{1} \frac{\partial T_{1}}{\partial x}\right|_{x=0}
$$

gives

$\frac{4 \pi}{b c}\left(k_{1}+k_{2}\right) \sum_{m=1 n=1}^{\infty} \sum_{n}^{\infty}\left(\frac{m^{2}}{b^{2}}+\frac{n^{2}}{c^{2}}\right)^{1 / 2} \cosh \pi a\left(\frac{m^{2}}{b^{2}}+\frac{n^{2}}{c^{2}}\right)^{1 / 2} \sin \frac{m \pi y}{b} \sin \frac{n \pi z}{c}$

$\int_{0}^{c} \int_{0}^{b} \phi\left(y^{\prime}, z^{\prime}\right) \sin \frac{m \pi y^{\prime}}{b} \sin \frac{n \pi z^{\prime}}{c} d y^{\prime} d z^{\prime} \mid$

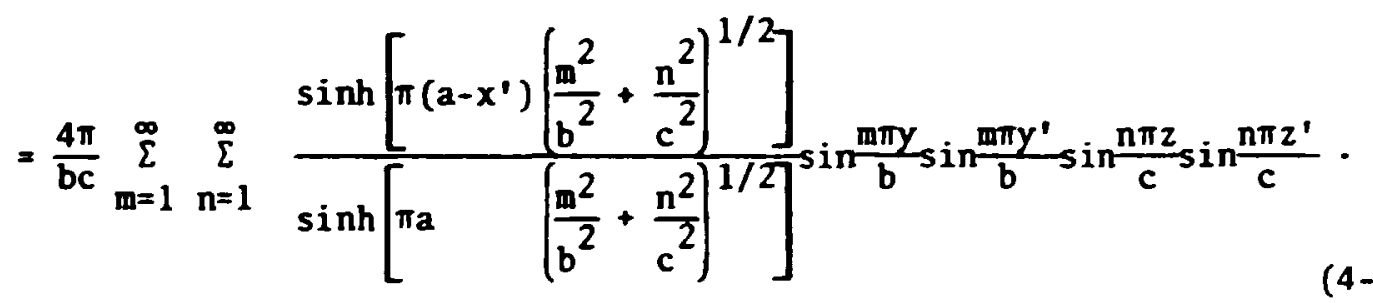

Assuming termwise equality gives

$\int_{0}^{c} \int_{0}^{b} \phi\left(y^{\prime}, z^{\prime}\right) \sin \frac{m \pi y^{\prime}}{b} \sin \frac{n \pi z^{\prime}}{c} d y^{\prime} d z^{\prime}$

$$
\left.=\frac{1}{\pi\left(k_{1}+k_{2}\right)\left(\frac{m^{2}}{b^{2}}+\frac{n^{2}}{c^{2}}\right)^{1 / 2}} \frac{\sinh \left[\pi\left(a-x^{\prime}\right)\left(\frac{m^{2}}{b^{2}}+\frac{n^{2}}{c^{2}}\right)^{1 / 2}\right]}{\cosh [\pi a}\left(\frac{m^{2}}{b^{2}}+\frac{n^{2}}{c^{2}}\right)^{1 / 2}\right]^{-i n} \frac{m \pi y^{\prime}}{b} \sin \frac{n \pi z^{\prime}}{c} .
$$

Using this in Equations (4-11) and (4-12) gives the solution as 


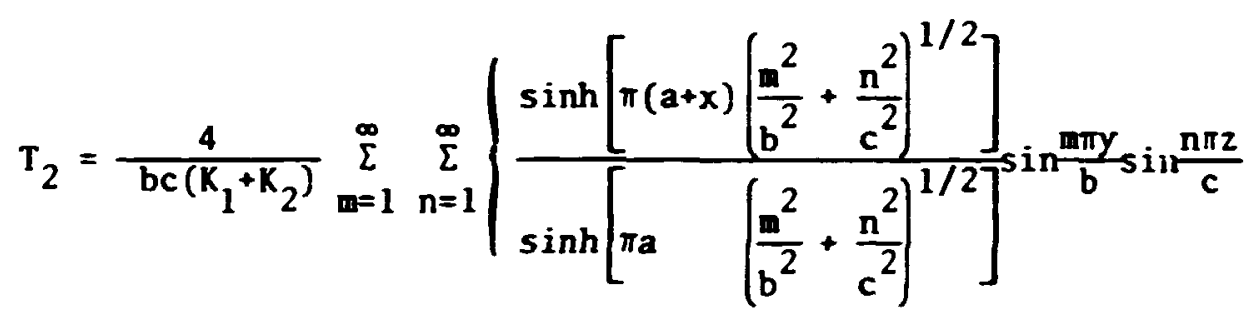

$$
\begin{aligned}
& \left.\left.\frac{1}{\left(\frac{m^{2}}{b^{2}}+\frac{n^{2}}{c^{2}}\right)^{1 / 2}} \frac{\sinh \left[\pi\left(a-x^{\prime}\right)\left(\frac{m^{2}}{b^{2}}+\frac{n^{2}}{c^{2}}\right)^{1 / 2}\right]}{\cosh [\pi a}\left(\frac{m^{2}}{\left(b^{2}\right.}+\frac{n^{2}}{c^{2}}\right)^{1 / 2}\right]^{\sin \frac{m \pi y^{\prime}}{b} \sin \frac{n \pi z^{\prime}}{c}}\right),
\end{aligned}
$$

and

$$
\begin{aligned}
& \left.T_{1}=\frac{4}{\pi b c\left(K_{1}+K_{2}\right)} \sum_{m=1 n=1}^{\infty} \sum_{\sum}^{\infty} \frac{1}{\left(\frac{m^{2}}{b^{2}}+\frac{n^{2}}{c^{2}}\right)^{1 / 2}} \frac{\sinh \left[\pi(a-x)\left(\frac{m^{2}}{b^{2}}+\frac{n^{2}}{c^{2}}\right)^{1 / 2}\right]}{\sinh [\pi a} \operatorname{(\frac {m^{2}}{b^{2}}+\frac {n^{2}}{c^{2}})^{1/2}}\right]^{\sin \frac{m \pi y}{b} \sin \frac{n \pi z}{c}} \\
& \left.\left.\frac{\sinh \left[\pi\left(a-x^{\prime}\right)\left(\frac{m^{2}}{b^{2}}+\frac{n^{2}}{c^{2}}\right)^{1 / 2}\right]}{\cosh [\pi a}\left(\frac{m^{2}}{b^{2}}+\frac{n^{2}}{c^{2}}\right)^{1 / 2}\right]^{\sin \frac{m \pi y^{\prime}}{b} \sin \frac{n \pi z^{\prime}}{c}}\right)+\frac{8}{k_{1} \pi^{2} a b c} \\
& \sum_{l=1 m=1}^{\infty} \sum_{n=1}^{\infty} \frac{1}{\frac{l^{2}}{a^{2}}+\frac{m^{2}}{b^{2}}+\frac{n^{2}}{c^{2}}} \sin \frac{\ell-\pi x}{a} \sin \frac{l \pi x^{\prime}}{a} \sin \frac{m \pi y}{b} \sin \frac{m \pi y^{\prime}}{b} \sin \frac{n \pi z}{c} \sin \frac{n \pi z^{\prime}}{c} .
\end{aligned}
$$

To show that these equations reduce to the one-media equations when $K_{1}=K_{2}$, consider an RPP $0 \leq x \leq 2 a, 0 \leq y \leq b, 0 \leq z \leq c$, with zero surface temperature and a point source at $\left(x^{\prime}, y^{\prime}, z^{\prime}\right)$. The steady-state solution (Equation $(4-6)$ ) is then 


$$
T=\frac{4}{K \pi^{2} a b c} \sum_{l=1 n=1 n=1}^{\infty} \sum_{\sum}^{\infty} \frac{1}{\frac{l^{2}}{4 a^{2}}+\frac{m^{2}}{b^{2}}+\frac{n^{2}}{c^{2}}} \sin \frac{l \pi x}{2 a} \sin \frac{l \pi x^{\prime}}{2 a} \sin \frac{m \pi y}{b} \sin \frac{n \pi y^{\prime}}{b} \sin \frac{n \pi z}{c} \sin \frac{n \pi z^{\prime}}{c},
$$

but

$$
\begin{aligned}
& \sum_{l=1}^{\infty} \frac{1}{\frac{l^{2}}{4 a^{2}}+\frac{m^{2}}{b^{2}}+\frac{n^{2}}{c^{2}}} \sin \frac{l \pi x}{2 a} \sin \frac{l \pi x^{\prime}}{2 a} \\
& =\frac{\pi a}{2\left(\frac{m^{2}}{b^{2}}+\frac{n^{2}}{c^{2}}\right)^{1 / 2}} \frac{\sinh \left[\pi x\left(\frac{m^{2}}{b^{2}}+\frac{n^{2}}{c^{2}}\right)^{1 / 2}\right] \sinh \left[\pi a\left(\frac{m^{2}}{b^{2}}+\frac{n^{2}}{c^{2}}\right)^{1 / 2}\right] \cosh \left[\pi a\left(2 a-x^{\prime}\right)\left(\frac{m^{2}}{b^{2}}+\frac{n^{2}}{c^{2}}\right)^{1 / 2}\right]}{\left.\left(\frac{m^{2}}{b^{2}}+\frac{n^{2}}{c^{2}}\right)^{1 / 2}\right]} ;
\end{aligned}
$$

therefore,

$$
\begin{aligned}
& T=\frac{2}{K \pi b c} \sum_{m=1 n=1}^{\infty} \sum_{\left(\frac{m^{2}}{b^{2}}+\frac{n^{2}}{c^{2}}\right)^{1 / 2}}^{\infty} \frac{\sinh \left[\pi\left(\frac{m^{2}}{\left(b^{2}\right.}+\frac{n^{2}}{c^{2}}\right)^{1 / 2}\right] \sinh \left[\pi\left(2 a-x^{\prime}\right)\left(\frac{m^{2}}{b^{2}}+\frac{n^{2}}{c^{2}}\right)^{1 / 2}\right]}{\sinh \left[\pi a\left(\frac{m^{2}}{b^{2}}+\frac{n^{2}}{c^{2}}\right)^{1 / 2}\right] \cosh \left[\pi a\left(\frac{m^{2}}{b^{2}}+\frac{n^{2}}{c^{2}}\right)^{1 / 2}\right]} \\
& \left.\cdot \sin \frac{m \pi y}{b} \sin \frac{m \pi y^{\prime}}{b} \sin \frac{n \pi z}{c} \sin \frac{n \pi z^{\prime}}{c}\right) \text {, }
\end{aligned}
$$

which is Equation (4-20) with $K_{1}=K_{2}=K$, and translated by a. 


\section{TWO-MEDIA RPP WITH SINUSOIDAL \\ INTERNAL SOURCE}

Problem 2: To calculate the temperature distribution within a three-dimensional, two media rectangular parallelepiped (Figure 3). Assume :

1. $K_{1}$ is the thermal conductivity for $0 \leq x \leq a, 0 \leq y \leq b$, $0 \leq z \leq c$

2. $k_{2}$ is the thermal conductivity for $-a \leq x \leq 0,0 \leq y \leq b$, $0 \leq z \leq c$

3. No contact resistance between media

4. All external surface temperatures are zero

5. Steady state

6. $S=S_{0} \sin \pi x / a \sin \pi y / b \sin \pi z / c$ for $0 \leq x \leq a, 0 \leq y \leq b$, $0 \leq z \leq c$

The analytical solution for this problem can be obtained usirg the previously generated Green's function, Equations (4-20) and (4-21).

Denoting the Green's function solution by $T_{g}\left(x, y, z, x^{\prime}, y^{\prime}, z^{\prime}\right)$ where $x^{\prime}, y^{\prime}, z^{\prime}$ is the source point, the temperature distribution due to a distributed source $S(x, y, z)$ is then given by

$$
T(x, y, z)=\iiint T_{g}\left(x, y, z, x^{\prime}, y^{\prime}, z^{\prime}\right) S\left(x^{\prime}, y^{\prime}, z^{\prime}\right) d x^{\prime} d y^{\prime} d z^{\prime} \quad .
$$

The solution for the Green's function in medium two (no source) was 


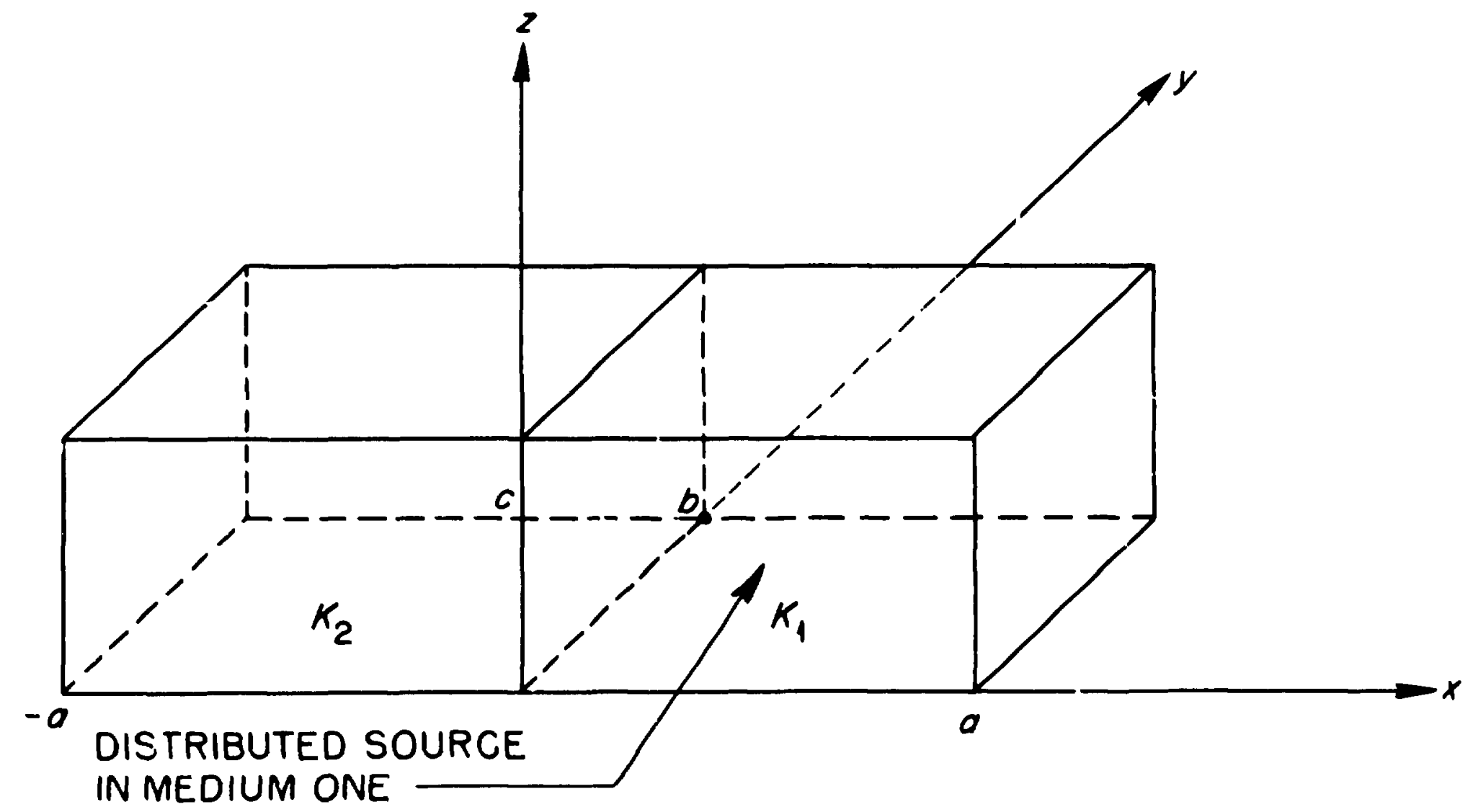

Figure 3. Two-media RPP with distributed source in mediun one. 


$$
\begin{aligned}
& T_{2 g}=\frac{4}{\pi b c\left(K_{1}+K_{2}\right)} \sum_{m=1 n=1}^{\infty} \sum_{\sinh [\pi a}^{\infty}\left(\frac{\sinh \left[\pi(a+x)\left(\frac{m^{2}}{b^{2}}+\frac{n^{2}}{c^{2}}\right)^{1 / 2} c^{2}\right)^{1 / 2}}{c^{2}}\right]^{\sin \frac{m \pi y}{b} \sin \frac{n \pi z}{c}} \frac{1}{\left(\frac{m^{2}}{b^{2}}+\frac{n^{2}}{c^{2}}\right)^{1 / 2}} \\
& \left.\frac{\sinh \left[\pi\left(a-x^{\prime}\right)\left(\frac{m^{2}}{b^{2}}+\frac{n^{2}}{c^{2}}\right)^{1 / 2}\right]}{\cosh [\pi a}\left[\left(\frac{m^{2}}{b^{2}}+\frac{n^{2}}{c^{2}}\right)^{1 / 2}\right]^{\sin \frac{m \pi y^{\prime}}{b} \sin \frac{n \pi z^{\prime}}{c}}\right) .
\end{aligned}
$$

Using the following integrals

$$
\int_{0}^{b} \sin \frac{\pi y^{\prime}}{b} \sin \frac{m \pi y^{\prime}}{b} d y^{\prime}= \begin{cases}b / 2, & m=1, \\ 0, & m \neq 1,\end{cases}
$$

$\int_{0}^{a} \sinh \left[\pi\left(a-x^{\prime}\right)\left(\frac{m^{2}}{b^{2}}+\frac{n^{2}}{c^{2}}\right)^{1 / 2}\right] \sin \frac{\pi x^{\prime}}{a} d x^{\prime}$

$$
=\sinh \left[\pi a\left(\frac{m^{2}}{b^{2}}+\frac{n^{2}}{c^{2}}\right)^{1 / 2}\right] \frac{a}{\pi+a^{2} \pi\left(\frac{m^{2}}{b^{2}}+\frac{n^{2}}{c^{2}}\right)},
$$

gives

$$
\begin{aligned}
& T_{2}(x, y, z)=\frac{S_{0} a}{\pi^{2}} \frac{1}{\left(K_{1}+K_{2}\right)} \frac{1}{1+a^{2}\left(\frac{1}{b^{2}}+\frac{1}{c^{2}}\right)} \\
& \frac{1}{\left(\frac{1}{b^{2}}+\frac{1}{c^{2}}\right)^{1 / 2}} \frac{\sinh \left[\pi(a-x)\left(\frac{1}{b^{2}}+\frac{1}{c^{2}}\right)^{1 / 2}\right]}{\cosh [\pi a} \sin \frac{\pi y}{b} \sin \frac{\pi z}{c} .
\end{aligned}
$$


The solution for the Green's function in medium one was

$$
\begin{aligned}
T_{1 g}\left(x, y, z, x^{\prime}, y^{\prime}, z^{\prime}\right) & =T_{2 g}\left(-x, y, z, x^{\prime}, y^{\prime}, z^{\prime}\right)+\frac{8}{K_{1} \pi^{2} a b c} \\
& \cdot \sum_{l=1 m=1 n=1}^{\infty} \sum_{\frac{l^{2}}{a^{2}}+\frac{m^{2}}{b^{2}}+\frac{n^{2}}{c^{2}}}^{\infty}\left(\frac{1}{c^{2}}\right) \\
& \left.\cdot \sin \frac{l \pi x}{a} \sin \frac{l \pi x^{\prime}}{a} \sin \frac{m \pi y}{b} \sin \frac{m \pi y^{\prime}}{b} \sin \frac{n \pi z}{c} \sin \frac{n \pi z^{\prime}}{c}\right) .
\end{aligned}
$$

Integrating Equation (4-30) gives

$T_{1}(x, y, z)=T_{2}(-x, y, z)+\frac{S_{0}}{\pi^{2} K_{1}} \frac{1}{\frac{1}{a^{2}}+\frac{1}{b^{2}}+\frac{1}{c^{2}}} \sin \frac{\pi x}{a} \sin \frac{\pi y}{b} \sin \frac{\pi z}{c}$.

To verify the solution, it is necessary that

$$
\begin{aligned}
& \nabla^{2} T_{2}(x, y, z)=0, \\
& \nabla^{2} T_{1}(x, y, z)=-\frac{s(x, y, z)}{K_{1}}, \\
& -\left.K_{1} \frac{\partial T_{1}}{\partial x}\right|_{x=0}=-\left.K_{2} \frac{\partial T_{2}}{\partial x}\right|_{x=0},
\end{aligned}
$$

and $T(x, y, z)=0$ for $x, y, z$ on outer boundaries. The verification of this solution is shown in Appendix $A$.

The parameters for the problem are:

1. $K_{1}=.1667 \mathrm{cal} / \mathrm{sec} \mathrm{cm}^{\circ} \mathrm{C}$

2. $\mathrm{K}_{2}=.3333 \mathrm{cal} / \mathrm{sec} \mathrm{cm}^{\circ} \mathrm{C}$ 
3. $a=1 \mathrm{~cm}$

4. $b=1 \mathrm{~cm}$

5. $c=1 \mathrm{~cm}$

6. $S_{0}=238.8(\pi / 2)^{3} \mathrm{cal} / \mathrm{sec} \mathrm{cm}^{3}$

with an external surface temperature of $80^{\circ} \mathrm{C}$.

This gives:

$T_{2}(x, y, z)=\frac{238.8 \pi}{12 \sqrt{2}} \frac{\sinh [\sqrt{2} \pi(1+x)]}{\cosh [\sqrt{2} \pi]} \sin \pi y \sin \pi z+80$,

for $-1 \leq x \leq 0,0 \leq y \leq 1,0 \leq z \leq 1$, and

$T_{1}(x, y, z)=\frac{238.8 \pi}{12 \sqrt{2}} \frac{\sinh [\sqrt{2} \pi(1-x)]}{\cosh [\sqrt{2} \pi]} y \sin z$

$+\frac{238}{4} \cdot 8 \pi \sin \pi x \sin \pi y \sin \pi z+80$,

for $0 \leq x \leq 1,0 \leq y \leq 1$, and $0 \leq z \leq 1$.

The source parameter $S_{0}$ is chosen to give a source of 1000 watts

$(238.8 \mathrm{cal} / \mathrm{sec})$ when the source is integrated over the source volume.

The Monte Carlo solution was attempted using the forward formulation and a next-event flux at a point estimator. The parameters for the transport equation solution were

1. $\Sigma_{1}=\frac{1}{3 \mathrm{~K}_{1}}=2 \mathrm{~cm}^{-1}$

2. $\Sigma_{2}=\frac{1}{3 K_{2}}=1 \mathrm{~cm}^{-1}$

3. $a=b=c=1 \mathrm{~cm}$

and the source $S$ is given by 


$$
S=238.8\left(\frac{\pi}{2}\right)^{3} \sin \pi x \sin \pi y \sin \pi z
$$

for $0 \leq x \leq 1,0 \leq y \leq 1,0 \leq y \leq 1$, and zero elsewhere.

Since these paraneters define a problem which is only one mean free path thick in one diension, scaling was necessary. A scaling factor of 10 was used. This gives:

$$
\Sigma_{1}=20 \mathrm{~cm}^{-1} \text {, }
$$

and

$$
\Sigma_{2}=10 \mathrm{~cm}^{-1} \text {, }
$$

and the correct temperature estimate $T$ is given by

$$
T=\frac{\phi}{10},
$$

where $\phi$ is the flux in units of $\mathrm{cm}^{-2} \mathrm{sec}^{-1}$ which is found using the scaled parameters. The results of performing a one-hour Monte Carlo calculation in the IBM $360 / 195$ are compared to the analytical resiles in Table 2. The temperature was calculated at $.1 \mathrm{~cm}$ intervals along the $y=.5 \mathrm{~cm}, 2=.5 \mathrm{~cm}$ centerline.

Although the results are not unreasonable, the standard deviations are high for a one-hour run on the IBM $360 / 195$. Since the standard deviations vary significantly from point to point, as for example, $1.52 \mathrm{C}^{\bullet}$ to $.34 \mathrm{C}^{\bullet}$ to $2.97 \mathrm{C}^{\circ}$ at $\mathrm{x}=-.7,-.6$, and -.5 , the possibility existed that the fluctuations and poor statistics were the result of using an unbounded estimator. 
TABLE 2

TEMPERATURE COMPARISONS FOR A TWO-MEDIA RPP WITH A SINUSOIDAL INTERNAL SOURCE USING THE FORWARD FORMULATION

\begin{tabular}{|c|c|c|c|}
\hline $\begin{array}{c}x \\
(\mathrm{~cm}) \\
\end{array}$ & $\begin{array}{c}\text { Analytic } \\
\text { Temperature } \\
{ }^{c} \mathrm{C} \\
\end{array}$ & $\begin{array}{l}\text { Monte Carlo } \\
\text { Temperature } \\
{ }^{\circ} \mathrm{C}\end{array}$ & $\begin{array}{c}\text { Escimated } \\
\text { Standard } \\
\text { Deviation } \\
C^{\circ}\end{array}$ \\
\hline-1.0 & 80.000 & 80.21 & .080 \\
\hline-.9 & 80.478 & 80.96 & .38 \\
\hline-.8 & 81.046 & 81.96 & .55 \\
\hline-.7 & 81.832 & 84.30 & 1.52 \\
\hline-.6 & 82.985 & 82.39 & .34 \\
\hline-.5 & 84.728 & 87.40 & 2.97 \\
\hline-.4 & 87.451 & 90.27 & 1.72 \\
\hline-.3 & 91.653 & 95.0 & 2.57 \\
\hline-.2 & 98.149 & 106.3 & 3.93 \\
\hline-.1 & 108.417 & 113.4 & 3.29 \\
\hline 0.0 & 124.178 & 132.5 & 7.82 \\
\hline .1 & 166.207 & 192. & 19.0 \\
\hline .2 & 208.713 & 195. & 12.7 \\
\hline .3 & 243.817 & 238 & 18.8 \\
\hline .4 & 266.025 & 264. & 15.8 \\
\hline .5 & 272.473 & 276. & 19.3 \\
\hline .6 & 261.488 & 254. & 15.2 \\
\hline .7 & 233.548 & 226. & 16.0 \\
\hline .8 & 191.042 & 195. & 10.7 \\
\hline .9 & 138.506 & 146.9 & 12.0 \\
\hline 1.0 & 80.000 & 91.5 & 1.37 \\
\hline
\end{tabular}


Wethods have been developed which produce a bounded next event flux at a point estinate, ${ }^{24-26}$ but these schemes are usually difficult to implement and require a nodification of the random walk procedure. To elininate these problems, a new bounded flux at a point estimator was developed.

For a monoenergetic flux, with isotropic scattering, the flux at a point $\mathbf{r}=0$, is given by

$\phi(r=0)=\int \Sigma_{s} \phi_{\text {avg }}(r) 4 \pi r^{2} \frac{e^{-\Sigma_{t} r}}{4 \pi r^{2}} d r+\int S(r) 4 \pi r^{2} \frac{e^{-\Sigma_{t} r}}{4 \pi r^{2}} d r$

where

$$
\phi_{\text {avg }}(r)=\frac{\iint_{A} \phi d A}{\int_{A} \int_{d A}}
$$

and $A$ is the surface area at $r$. A Monte Carlo estimate of Equation (4-41) is obtained using each collision as an estimate of $\sum_{s} \phi_{\text {avg }}(r) 4 \pi r^{2}$ and scoring $e^{-\sum_{t} r} / 4 \pi r^{2}$ at each collision. The source term may be added on analytically or $e^{-\Sigma_{t} r} / 4 \pi r^{2}$ is scored at each source location chosen.

The large or unbounded estimates occur from collisions very near the point at which the flux is being estimated. In this case the $e^{-\Sigma_{t} r} / 4 \pi r^{2}$ contribution is very large. (Note, however, that the total integral is bounded since the $r^{2}$ terms cancel.) 
To eliminate this problem, consider the contributions to the thux estimator from collisions which occur within a sphere of radius ' $a$ ' about the point

$$
C=\int_{0}^{a} \Sigma_{s} \phi_{a v_{g}}(r) 4 \pi r^{2} \frac{e^{-\Sigma_{\tau} r}}{4 \pi r^{2}} d r
$$

If $\operatorname{davg}(r)$ is known, then a ' $d$ ' can be calculated such that

$$
\int_{0}^{a} \Sigma_{s} \phi_{a v g}(r) 4 \pi r^{2} \frac{e^{-\Sigma_{t} r}}{4 \pi r^{2}} d r=\int_{0}^{a} \Sigma_{s} \operatorname{avg}(r) 4 \pi r^{2} \frac{e^{-\Sigma_{t} r}}{4 \pi d^{2}} d r
$$

where $e^{-\Sigma_{t} r} / 4 \pi d^{2}$ is now scored at each collision which occurs within radius ' $a$ '. This estimate is bounded and gives the correct estimate. But, the calculation of the correct ' $d$ ' depends upon knowing $\phi_{\text {avg }}(r)$. For spheres with a radius ' $a$ ' up to a mean free path, ' $d$ ' is fairly independent of the flux shape within the sphere. Calculations of ' $d$ ' are performed in Appendix B for a flux which varies linearly across the sphere and for a flux which varies exponentially across the sphere.

Using these results, the calculation of the temperat:ire at a dofint was performed using several different size spheres to bound the estir.ator. The results of these calculations are shown in Table 3. These calculations are of the temperature at $(.1, .5, .5)$, and were made using 4.5 min of CPU time on the IBM 360/91. The analytical result for this point is $16 i .20 \%^{\circ} \mathrm{C}$.

These calculations demonstrate that the poor statistics were not primarily the result of using an unbounded estimator. No collisions 
TABLE 3

TEMPERATURES AT A POINT USING A BOUNDED NEXT EVENT ESTIMATOR

\begin{tabular}{|c|c|c|c|c|}
\hline $\begin{array}{l}\text { Bounding Radius } \\
\text { (mean free paths) }\end{array}$ & $\begin{array}{c}\text { Total } \\
\text { Response } \\
{ }^{\circ} \mathrm{C} \\
\end{array}$ & $\begin{array}{l}\text { Estimated } \\
\text { Standard } \\
\text { Deviat ion } \\
\mathrm{C}^{\bullet} \\
\end{array}$ & $\begin{array}{c}\text { Response in } \\
\text { Bounding Sphere } \\
{ }^{\circ} \mathrm{C}\end{array}$ & $\begin{array}{l}\text { Collisions* in } \\
\text { Bounding Sphere }\end{array}$ \\
\hline 0.0 & 160.7 & 17.7 & 0.0 & 0 \\
\hline 0.1 & 160.7 & 17.7 & 0.0 & 0 \\
\hline 0.25 & 176.0 & 26.4 & 34.4 & 3 \\
\hline 0.50 & 167.9 & 14.9 & 38.0 & 15 \\
\hline 1.00 & 159.8 & 9.6 & 46.3 & 86 \\
\hline 2.00 & 167.2 & 7.9 & 74.0 & $87 n$ \\
\hline
\end{tabular}

*There was a total of 151,387 collisions in the problem. 
occurred within .1 mean free path of the point detector and only 870 out of 151387 collisions occurred within two mean free paths. Note, however, that the collisions within two mean free paths contribute approximately 858 of the answer due to collisions $\left(80 \mathrm{C}^{\circ}\right.$ of the answer is due to the external boundary condition) and that collisions within one mean free path contributes over 50 of the answer due to collisions. The poor statistics are a result then of not having a sufficient number of collisions in the area of interest. These results suggest that the adjoint formulation would be more appropriate for this problem.

In the adjoint formulation the forward response becomes the adjoint source (all particles are started at the point of interest) and the forward source becomes the adjoint response (a collision density estimator was used to score the source at each collision site). With these changes a Monte Carlo calculation of the temperature at $(.1, .5$, .5) was made using 4.5 min of CPU time on the IBM 360/91. The answer obtained was $175.0^{\circ} \mathrm{C}$ with an estimated standard deviation of $1.52 \mathrm{C}^{\circ}$. The standard deviation is approximately a factor of 10 lower than the forward calculation using the same computing time. A reduction of the standard deviation by a factor of 10 is roughly equivalent to a factor of 100 in reduction of computing time. The disadvantage of the adjoint calculation is that a separate computer run must be made for each point at which a temperature calculation is to be made.

Adjoint calculations were made for the temperatures at four points along the $y=.5, z=.5$ centerline. The results are shown in Table 4. Each calculation represents 4.5 min of IBM 360/91 CPU time. 
TABLE 4

TEMPERATURE COMPARISONS FOR A TWO-MEDIA RPP WITH A SINUSOIDAL INTERNAL SOURCE USING THE ADJOINT FORMULATION

\begin{tabular}{cccc}
\hline $\begin{array}{c}\text { Analytic } \\
(\mathrm{cm})\end{array}$ & $\begin{array}{c}\text { Temperature } \\
{ }^{\circ} \mathrm{C}\end{array}$ & $\begin{array}{c}\text { Monte Carlo } \\
\text { Temperature } \\
{ }^{\circ} \mathrm{C}\end{array}$ & $\begin{array}{c}\text { Est imated } \\
\text { Standard } \\
\text { Deviation } \\
\mathrm{C}^{\circ}\end{array}$ \\
\hline. .7 & 81.83 & 82.75 & .18 \\
-.5 & 84.73 & 86.50 & .31 \\
.1 & 166.2 & 175.0 & 1.52 \\
.5 & 272.5 & 284.3 & 3.94 \\
\hline
\end{tabular}


The Monte Carlo results are high. The magnitude of the error is that expected due to the edge effects (not including an extrapolation distance sorrection). The effects of not including this correction factor and ways of minimizing this error are discussed in more detail in two-media right circular cylinder problems later in the section, in which case deterministic computer codes could be used to calculate the transport theory solution and therefore eliminate the uncertainty associated with the Monte Carlo results. One method to reduce the error associated with the edge effects is to increase the scale factor used in the calculations. The calculations of the temperature at $(.1, .5, .5)$ were performed using a scale factor of 40 . The calculation represents $20 \mathrm{~min}$ of CPU time on the IBM 360/195. The Monte Carlo result was $171.0^{\circ} \mathrm{C}$ with an estimated standard deviation of $2.05 \mathrm{C}^{\circ}$. An even better answer would be obtained if a larger scale factor (requiring more CPU time) was used.

IV. TWO-MEDIA KPP WITH IINIFOKM INTIRNAL SOURCI:

Problem 3: To calculate the temperature distribution in a threedimensional, two media-rectangular parallelepiped as in the previous problefu except with a source distribution of

$$
g(x, y, z)=23 x .8 \mathrm{cal} / \mathrm{sec} \mathrm{cm}^{3},
$$

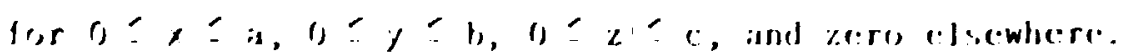

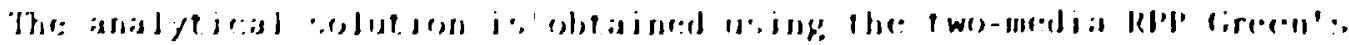
fuspre.tiche. 
Using the following integrals

$$
\int_{0}^{b} \sin \frac{m \pi y}{b} d y= \begin{cases}\frac{2 b}{m \pi}, & \text { m odd }, \\ 0, & \text { m even }\end{cases}
$$

and

$$
\int_{0}^{a} \sinh \left[\pi(a-x)\left(\frac{m^{2}}{b^{2}}+\frac{n^{2}}{c^{2}}\right)^{1 / 2}\right] d x=\frac{\cosh \left[\pi a\left(\frac{m^{2}}{b^{2}}+\frac{n^{2}}{c^{2}}\right)^{1 / 2}\right]-1}{\pi\left(\frac{m^{2}}{b^{2}}+\frac{n^{2}}{c^{2}}\right)^{1 / 2}},
$$

gives

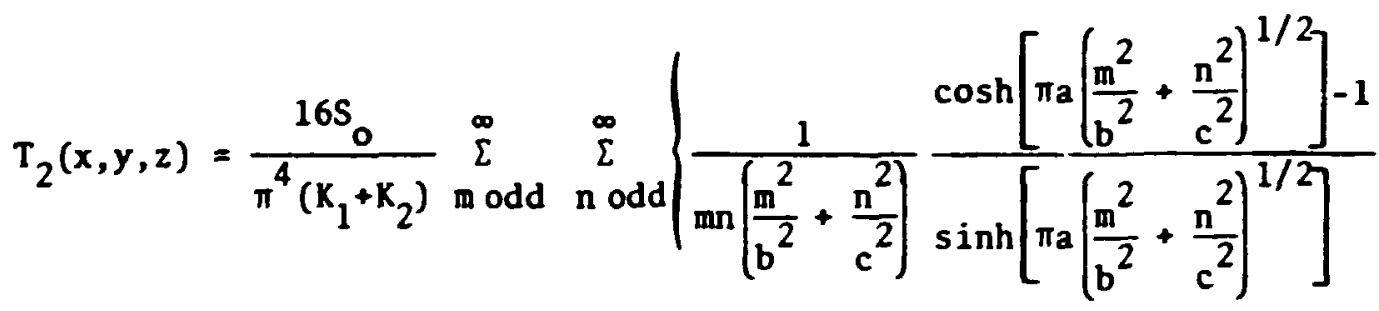

$$
\begin{aligned}
& \left.\left.\frac{\sinh \left[\pi(a+x)\left(\frac{m^{2}}{b^{2}}+\frac{n^{2}}{c^{2}}\right)^{1 / 2}\right]}{\cosh :\left[\pi a\left(\frac{m^{2}}{b^{2}}+\frac{n^{2}}{c^{2}}\right)^{1 / 2}\right.}\right]^{\sin \frac{m \pi y}{b} \sin \frac{n \pi z}{c}}\right)
\end{aligned}
$$

and

$$
\begin{aligned}
& T_{1}(x, y, z)=T_{2}(-x, y, z)+\frac{64 S_{o}}{k_{1} \pi^{5}} \sum_{\ell \text { odd }}^{\infty} \sum_{m}^{\infty} \sum_{\substack{\text { odd } \\
n \text { odd }}}^{\infty} \frac{1}{\operatorname{lmn}\left(\frac{l^{2}}{a^{2}}+\frac{m^{2}}{b^{2}}+\frac{n^{2}}{c^{2}}\right)} \\
& \text { - } \left.\sin \frac{\ell \pi x}{a} \sin \frac{\pi \pi y}{b} \sin \frac{\pi \pi z}{c}\right\} \text {. }
\end{aligned}
$$

This solution is verified in Appendix A. 
This solution still requires the evaluation of an infinite series to obtain numerical results. However, the series is absoluteiy convergent and converges very quickly.

The Morte Carlo solution was performed using the adjoint formulation. A scaling factor of 10 was used. Adjoint calculations were made for the temperature at four points along the $y=.5, z=.5$ centerline. The results are shown in Table 5. Each Monte Carlo calculation represents $4.5 \mathrm{~min}$ of IBM $360 / 91$ CPU time.

\section{TWO-MEDIA RPP WITH GIVEN SURFACE TEMPERATURE DISTRIBUTION}

Problem 4: To calculate the temperature distribution in a three-dimensional, two media rectangular parallelepiped as in the previous próblem except:

1. No internal sources

2. $T(x=a)=\sin \pi y / b \sin \pi z / c$.

The analytical solution is obtained using the two-media RPP Green's function. Denoting the Green's function solution by $T_{g}\left(x, y, z, x^{\prime}, y^{\prime}, z^{\prime}\right)$ where $\left(x^{\prime}, y^{\prime}, z^{\prime}\right)$ is the source point, the temperature distribution due to a surface temperature $\phi(x, y, z)$ is given by

$$
T\left(x^{\prime}, y^{\prime}, z^{\prime}\right)=K \iint_{S}^{\partial T_{g}} \frac{g}{\partial n}\left(x, z, x^{\prime}, y^{\prime}, z^{\prime}\right) \phi(x, y, z) d x d y d z
$$

where $\partial / \partial$ n denotes differentiation with respect to the $x, y, z$ variables along the inward normal to the surface. 
TABLE 5

TEMPERATURE COMHARISONS FOR A THC-YEDIA RFF WITH A UNIFORMI INTER.IAL SN'JRCE

\begin{tabular}{|c|c|c|c|}
\hline $\begin{array}{c}x \\
(\mathrm{~cm}) \\
\end{array}$ & $\begin{array}{l}\text { Analytic } \\
\text { Temperature } \\
\text { oc }\end{array}$ & $\begin{array}{l}\text { Monte Carlo } \\
\text { Temperature } \\
{ }^{\circ} \mathrm{C}\end{array}$ & $\begin{array}{l}\text { Estimated } \\
\text { standing } \\
\text { noviation } \\
\end{array}$ \\
\hline-.7 & $81.5 \mathrm{~s}$ & $\$ 2.5$ & .12 \\
\hline-.5 & 54.07 & $s(1.0)$ & $\therefore 1$ \\
\hline .1 & 1.30 .67 & $1+5.2$ & .5 \\
\hline .5 & 164.61 & $1-4.5$ & $\therefore$ \\
\hline
\end{tabular}


Performing the indicated integration using the followin: integral:

$$
\int_{0}^{b} \sin \frac{\pi y}{b} \sin \frac{m \pi y}{b} d y= \begin{cases}b / 2, & m=1, \\ 0, & m \neq 1,\end{cases}
$$

and the following Fourier expansion:

$$
\frac{2}{\pi} \sum_{\ell=1}^{\infty} \frac{l(-1)^{l} \sin \frac{l \pi x}{a}}{l^{2}+a^{2}\left(\frac{1}{b^{2}}+\frac{1}{c^{2}}\right)}=-\frac{\sinh \pi x\left(\frac{1}{b^{2}}+\frac{1}{c^{2}}\right)^{1 / 2}}{\sinh \pi a\left(\frac{1}{b^{2}}+\frac{1}{c^{2}}\right)^{1 / 2}},
$$

gives

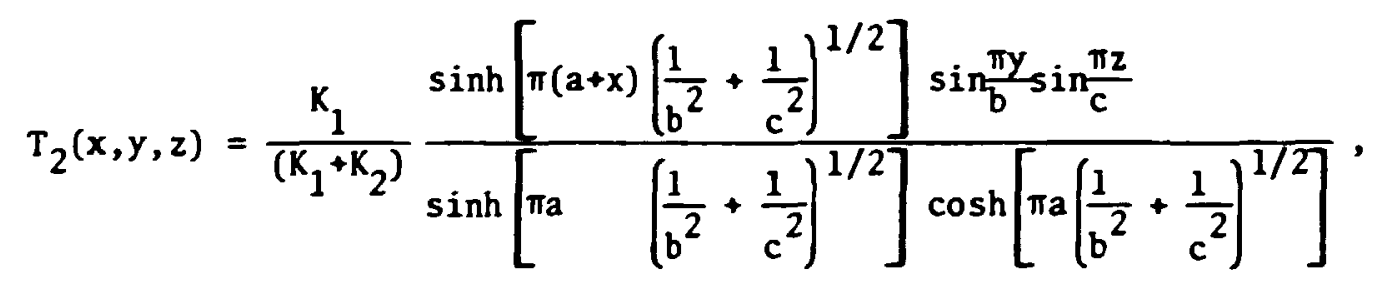

for $-a \leq x \leq 0,0 \leq y \leq b, 0 \leq z \leq c$

and

$$
T_{1}(x, y, z)=T_{2}(-x, y, z)+\frac{\sinh \pi x\left(\frac{1}{b 2}+\frac{1}{c}\right)^{1 / 2}}{\sinh \pi a\left(\frac{1}{b^{2}}+\frac{1}{c^{2}}\right)^{1 / 2} \sin \frac{\pi y}{b} \sin \frac{\pi z}{c},}
$$

for $0 \leq x \leq a, 0 \leq y \leq b$, and $0 \leq z \leq c$.

This solution is verified in Appendix A. For numerical results a temperature of $80^{\circ} \mathrm{C}$ was used on all external boundaries except for the face at $x=1$ which was maintained at $T(x=1)=2200 \sin \pi y \sin \pi z+80$. 
The Monte Carlo solutions were perforned using the adjoint formulation. The external temperature was scored for each particle which escaped. A scaling factor of 10 was used. Adjoint calculations were made for the temeratures at four points along the $y=.5, z=.5$ centerline. The results of the Monte Carlo solutions are compared to the analytical results in Table 6. Each Monte Carlo calculation was made using 4.5 min of CPU time on the IBM 360/91.

\section{THREE-MEDIA RPP GREEN'S FUNCTION}

To solve the next problem, it is first necessary to derive a Green's function for a three-media rectangular parallelepiped (Figure 4).

The same geometry as used in the two-media rectangular parallelepiped Green's function will be retained with an additional medium having a conductive coefficient $K_{3}$ added. This gives $K_{2}$ for $-a \leq x \leq 0,0 \leq y \leq b, 0 \leq z \leq c, k_{1}$ for $0 \leq x \leq a, 0 \leq y \leq b$, $0 \leq z \leq c$, and $k_{3}$ for $a \leq x \leq 2 a, 0 \leq y \leq b$, and $0 \leq z \leq c$.

The Green's function will be calculated for a point source in the medium with conductive coefficient $K_{1}$. Assume that the temperature at the interface at $x=0$ is given by $\phi(y, z)$ and the temperature at the interface at $x=a$ is given by $\psi(y, z)$. Assuming no contact resistance at media interfaces and that all external boundaries are at zero temperatures, the temperatures in the three media can then be obtained from the single medium Green's function and are given by: 
TABLE 6

TEMPERATURE COMPARISONS FOR A TWO-MEDIA RPP WITH A GIVEN SURFACE TEMPERATURE DISTRIBUTION

\begin{tabular}{cccc}
\hline $\begin{array}{c}\text { Analytic } \\
(\mathrm{cm})\end{array}$ & $\begin{array}{c}\text { Monte Carlo } \\
\text { Temperature } \\
{ }^{\circ} \mathrm{C}\end{array}$ & $\begin{array}{c}\text { Temperature } \\
{ }^{\circ} \mathrm{C}\end{array}$ & $\begin{array}{c}\text { Estimated } \\
\text { Standard } \\
\text { Deviation } \\
\mathbf{C}^{\circ}\end{array}$ \\
\hline-.7 & 80.716 & 80.94 & .25 \\
-.5 & 81.849 & 82.86 & .51 \\
.1 & 114.826 & 117.3 & 3.17 \\
\hline
\end{tabular}




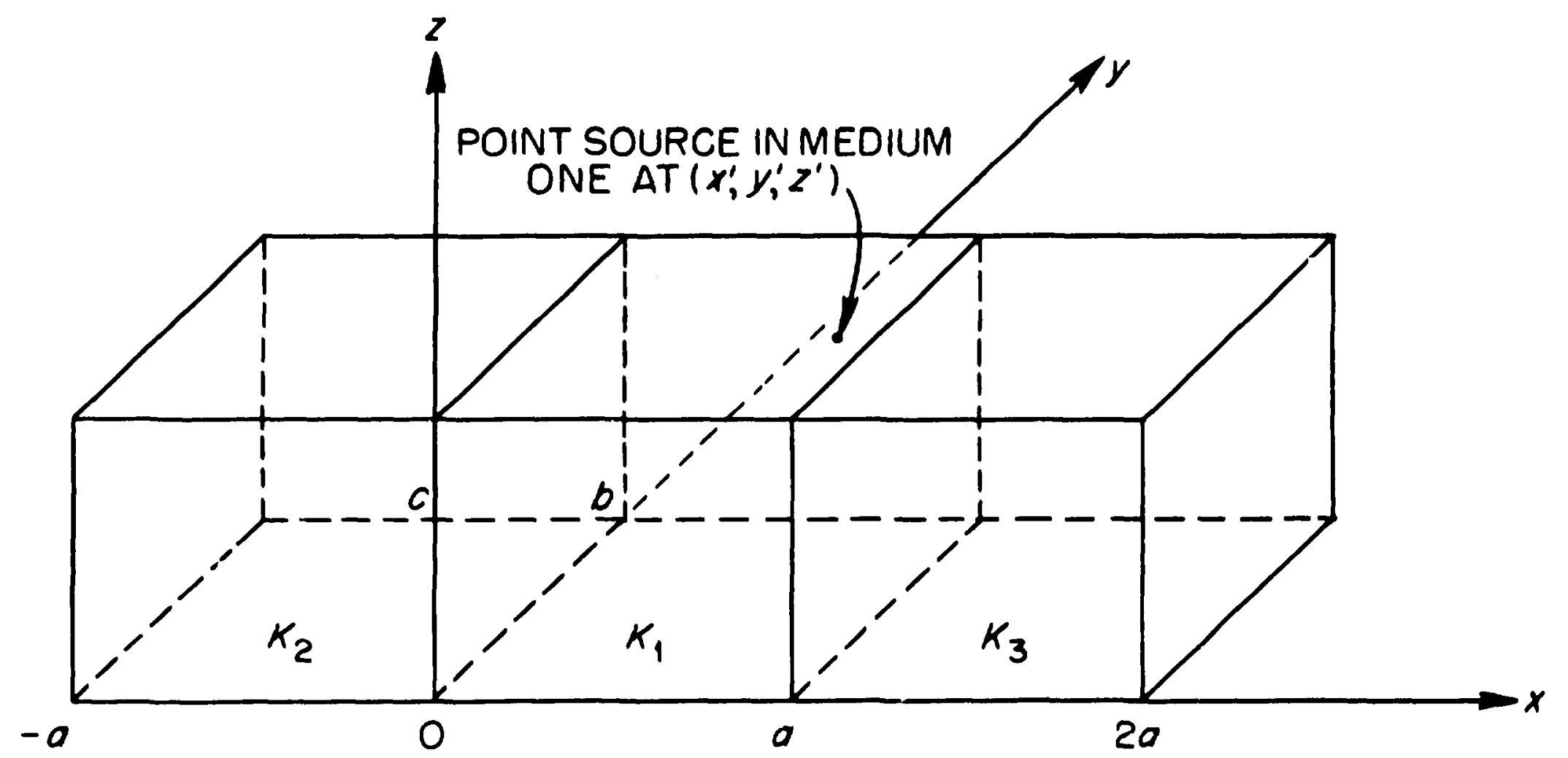

Figure 4. Three-media RPP with point solrce in medium one. 


$$
\begin{aligned}
& T_{2}(x, y, z)=\frac{4}{b c} \sum_{m=1 n=1}^{\infty} \sum_{m=1}^{\infty} \frac{\sinh \left[\pi(a+x)\left(\frac{a^{2}}{b^{2}}+\frac{n^{2}}{c^{2}}\right)^{1 / 2}\right]}{\sinh [\pi a} \frac{\left(\frac{m^{2}}{b^{2}}+\frac{n^{2}}{c^{2}}\right)^{1 / 2}}{\sin \frac{m \pi y}{b} \sin \frac{n \pi z}{c}} \\
& \text { - } \left.\int_{0}^{c} \int_{0}^{b} \phi\left(y^{\prime}, z^{\prime}\right) \sin \frac{m \pi y^{\prime}}{b} \sin \frac{n \pi z^{\prime}}{c} d y^{\prime} d z^{\prime}\right) \text {, } \\
& \text { for }-a \leq x \leq 0,0 \leq y \leq b, 0 \leq z \leq c \\
& T_{3}(x, y, z)=\frac{4}{b c} \sum_{m=1 n=1}^{\infty} \sum_{\sinh [\pi a}^{\infty}\left(\frac{\left.\sinh \left[\pi(2 a-x)\left(\frac{m^{2}}{b^{2}}+\frac{n^{2}}{c^{2}}\right)^{1 / 2}+\frac{n^{2}}{c^{2}}\right)^{1 / 2}\right]}{\sin \frac{m \pi}{b} \sin \frac{n \pi z}{c}}\right. \\
& \text { - } \left.\int_{0}^{c} \int_{0}^{b} \psi\left(y^{\prime}, z^{\prime}\right) \sin \frac{m \pi y^{\prime}}{b} \sin \frac{n \pi z^{\prime}}{c}-d y^{\prime} d z^{\prime}\right) \text {, }
\end{aligned}
$$

for $a \leq x \leq 2 a, 0 \leq y \leq b, 0 \leq z \leq c$,

and

$$
\begin{aligned}
& T_{1}(x, y, z)=\frac{4}{b c} \sum_{m=1 n=1}^{\infty} \sum_{\sinh [\pi a}^{\infty}\left\{\frac{\sinh \left[\pi(a-x)\left(\frac{m^{2}}{b^{2}}+\frac{n^{2}}{c^{2}}\right)^{1 / 2}\right]}{\left(\frac{m^{2}}{b^{2}}+\frac{n^{2}}{c^{2}}\right)^{1 / 2}}\right]^{\sin \frac{m \pi y}{b} \sin \frac{n \pi z}{c}} \\
& \cdot \int_{0}^{c} \int_{0}^{b} \phi\left(y^{\prime}, z^{\prime}\right) \sin \frac{m \pi y^{\prime}}{b} \sin \frac{n \pi z^{\prime}}{c} d y^{\prime} d z^{\prime} \\
& +\frac{4}{b c} \sum_{m=1}^{\infty} \sum_{\sum=1}^{\infty}\left(\frac{\sinh \pi x\left(\frac{m^{2}}{b^{2}}+\frac{n^{2}}{c^{2}}\right)^{1 / 2}}{\sinh \pi a\left(\frac{m^{2}}{b^{2}}+\frac{n^{2}}{c^{2}}\right)^{1 / 2} \sin \frac{m \pi y}{b} \sin \frac{n \pi z}{c}}\right.
\end{aligned}
$$




$$
\begin{aligned}
& \cdot \int_{0}^{c} \int_{0}^{b} \psi\left(y^{\prime} z^{\prime}\right) \sin \frac{m \pi y^{\prime}}{b} \sin \frac{n \pi z^{\prime}}{c} d y^{\prime} d z^{\prime} \mid+\frac{8}{k_{1} \pi^{2} a b c} \\
& \cdot \sum_{l=1 m=1 n=1}^{\infty} \sum_{\sum}^{\infty} \frac{1}{\frac{l^{2} a^{2} n^{2}}{a^{2} b^{2} c^{2}}} \sin \frac{l \pi x}{a} \sin \frac{l \pi x^{\prime}}{a} \sin \frac{m i y}{b} \sin \frac{m \pi y^{\prime}}{b} \sin \frac{n \pi z}{c} \sin \frac{n \pi z^{\prime}}{c},
\end{aligned}
$$

for $0 \leq x \leq a, 0 \leq y \leq b, 0 \leq z \leq c$,

where $\phi(y, z)$ and $\psi(y, z)$ are still to be determined. At the interface at $x=0$ continuity of heat flow gives

$$
\left.K_{2} \frac{\partial T_{2}}{\partial x}\right|_{x=0}=\left.k_{1} \frac{\partial T_{1}}{\partial x}\right|_{x=0}
$$

Solving for $\left.\frac{\partial T_{2}}{\partial x}\right|_{x=0}$ gives

$$
\begin{aligned}
\left.\frac{\partial T}{\partial x}\right|_{x=0}= & \frac{4 \pi}{b c} \sum_{m=1 n=1}^{\infty} \sum_{\left(b^{2}\right.}^{\infty}\left(\frac{m^{2}}{c^{2}}+\frac{n^{2}}{c^{2}}\right)^{1 / 2} \frac{\cosh \pi a\left(\frac{m^{2}}{b^{2}}+\frac{n^{2}}{c^{2}}\right)^{1 / 2}}{\sinh \pi a\left(\frac{m^{2}}{b^{2}}+\frac{n^{2}}{c^{2}}\right)^{1 / 2} \sin \frac{n \pi y}{b} \sin \frac{n \pi z}{c}} \\
& \left.\cdot \int_{0}^{c} \int_{0}^{b} \phi\left(y^{\prime}, z^{\prime}\right) \sin \frac{m \pi y^{\prime}}{b} \sin \frac{n \pi z^{\prime}}{c} d y^{\prime} d z^{\prime}\right)
\end{aligned}
$$

For simplicity of notation the integral term will be denoted $F(m, n)$.

Solving for $\left.\frac{\partial T_{1}}{\partial x}\right|_{x=0}$ gives 


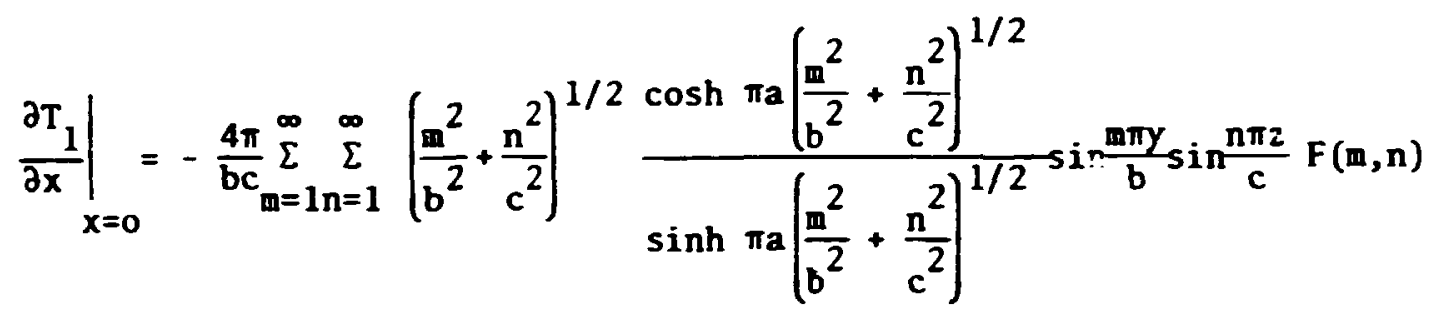

$$
\begin{aligned}
& +\frac{4 \pi}{b c} \sum_{m=1 n=1}^{\infty} \sum_{\sum}^{\infty}\left(\frac{m^{2}}{b^{2}}+\frac{n^{2}}{c^{2}}\right)^{1 / 2} \frac{\sin \frac{m \pi y}{b} \sin \frac{n \pi z}{c}}{\sinh \pi a\left(\frac{m^{2}}{b^{2}}+\frac{n^{2}}{c^{2}}\right)^{1 / 2}} \\
& \cdot \int_{0}^{c} \int_{0}^{b} \psi\left(y^{\prime}, z^{\prime}\right) \sin \frac{m \pi y^{\prime}}{b} \sin \frac{n \pi z^{\prime}}{c} d y^{\prime} d z^{\prime} \mid+\frac{4 \pi}{K_{1} b c}
\end{aligned}
$$

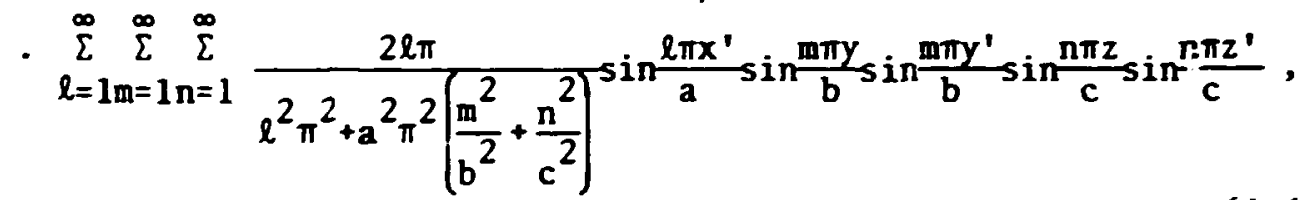

where the remaining integral term will be denoted $G(m, n)$.

Using

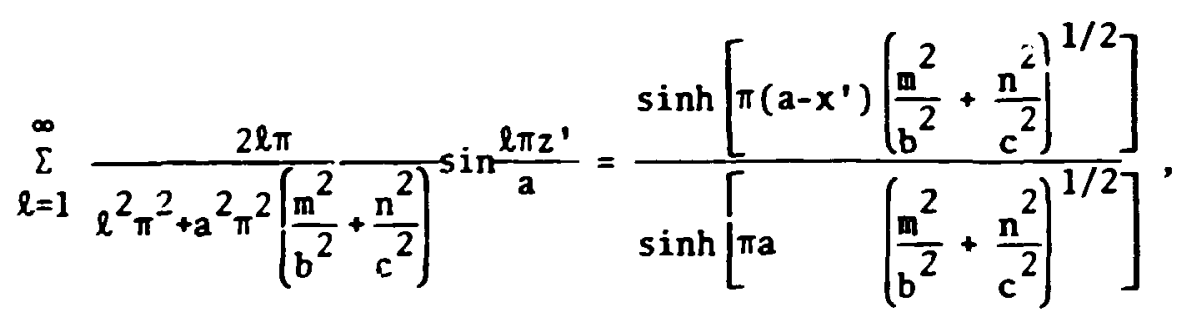

and setting $\left.k_{2} \frac{\partial T_{2}}{\partial x}\right|_{x=0}=\left.K_{1} \frac{\partial T_{1}}{\partial x}\right|_{x=0}$ gives 


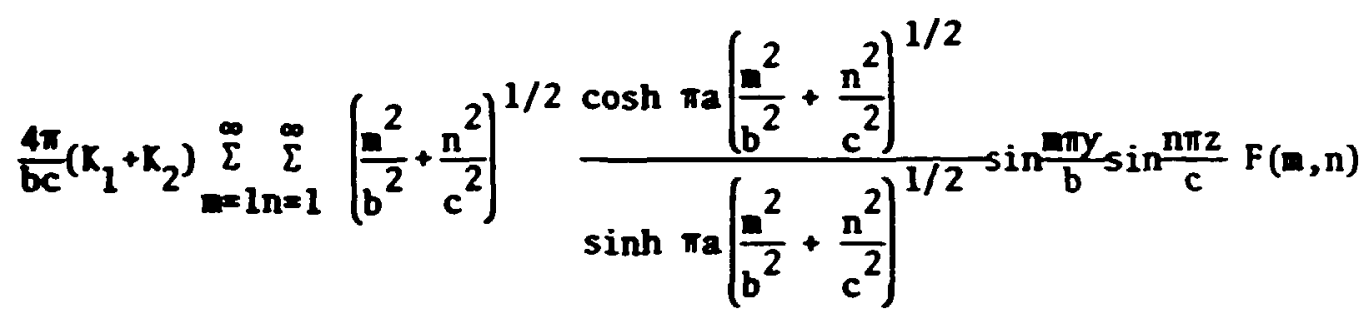

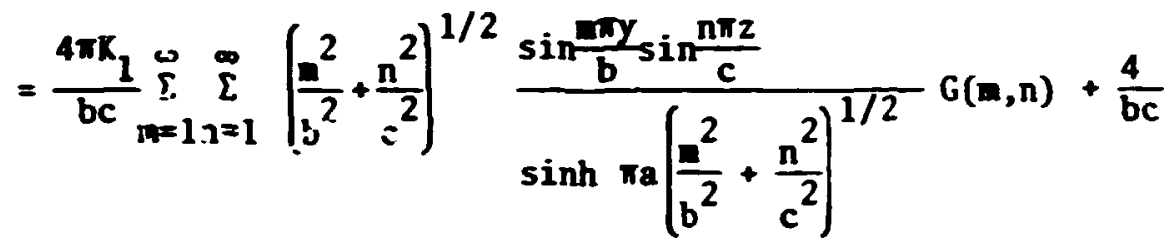

- $\left.\sum_{n=1 n=1}^{\infty} \sum_{\sinh [\pi a}^{\sinh \left[\pi\left(a-x^{\prime}\right)\left(\frac{n^{2}}{b^{2}}+\frac{n^{2}}{c^{2}}\right)^{1 / 2}\right]}\left(\frac{n^{2}}{b^{2}}+\frac{n^{2}}{c^{2}}\right)^{1 / 2}\right]^{\sin \frac{n \pi y}{b} \sin \frac{m \pi y^{\prime}}{b} \sin \frac{n \pi z}{c} \sin \frac{n \pi z^{\prime}}{c}}$.

For termwise equality, which follows from the orthogonality of sine functions,

$$
\begin{aligned}
& \pi\left(K_{1}+K_{2}\right)\left(\frac{a^{2}}{b^{2}}+\frac{n^{2}}{c^{2}}\right)^{1 / 2} \cosh \pi a\left(\frac{m^{2}}{b^{2}}+\frac{n^{2}}{c^{2}}\right)^{1 / 2} F(m, n) \\
& =\pi K_{1}\left(\frac{m^{2}}{b^{2}}+\frac{n^{2}}{c^{2}}\right)^{1 / 2} G(m, n)+\sinh \pi\left(a-x^{\prime}\right)\left(\frac{m^{2}}{b^{2}}+\frac{n^{2}}{c^{2}}\right)^{1 / 2} \sin \frac{m \pi y^{\prime}}{b} \sin \frac{n \pi z^{\prime}}{c}
\end{aligned}
$$

For continuity of heat flow at the interface at $x=a$

$$
\left.K_{1} \frac{\partial T_{1}}{\partial x}\right|_{x=a}=\left.K_{3} \frac{\partial T_{3}}{\partial x}\right|_{x=a} .
$$


Solving for $\left.\frac{\partial T_{3}}{\partial x}\right|_{x=a}$ gives

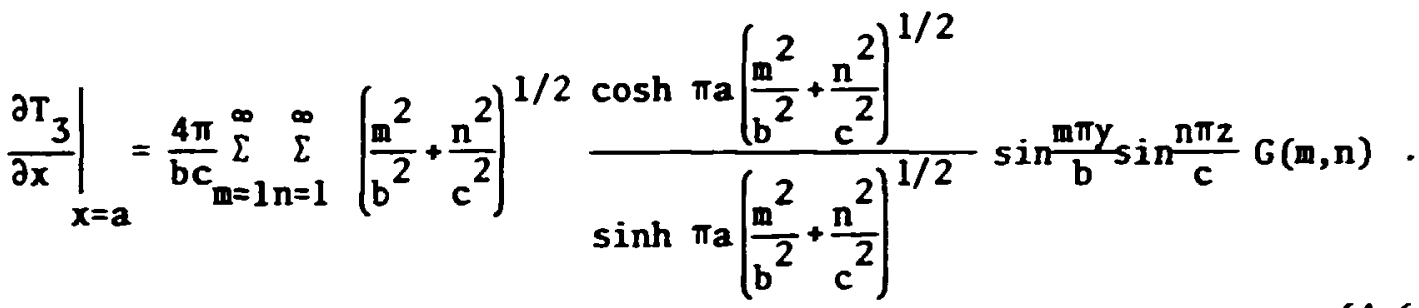

Solving for $\left.\frac{\partial T_{1}}{\partial x}\right|_{x=a}$ gives

$$
\begin{aligned}
& \left.\frac{\partial T}{\partial x}\right|_{x=a}=-\frac{4 \pi}{b c} \sum_{m=1 n=1}^{\infty} \sum_{b^{2}}^{\infty}\left(\frac{m^{2}}{b^{2}}+\frac{n^{2}}{c^{2}}\right)^{1 / 2} \frac{\sin \frac{m \pi y}{b} \sin \frac{n \pi z}{c}}{\sinh \pi a\left(\frac{m^{2}}{b^{2}}+\frac{n^{2}}{c^{2}}\right)^{1 / 2}} F(m, n)+\frac{4 \pi}{b c}
\end{aligned}
$$

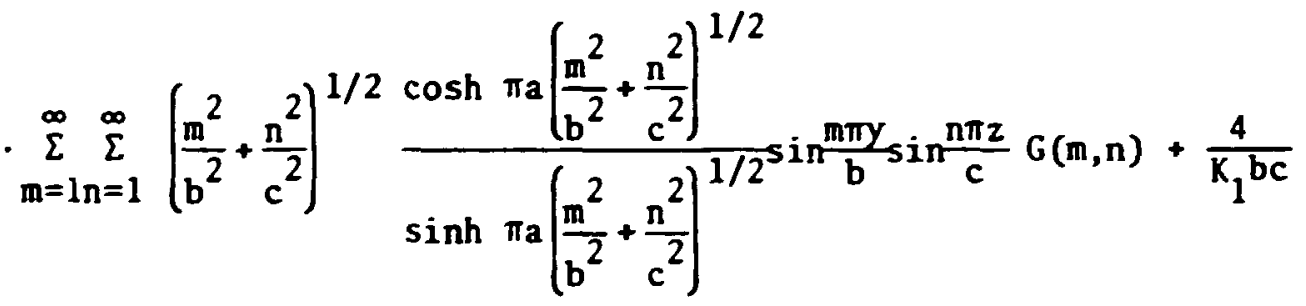

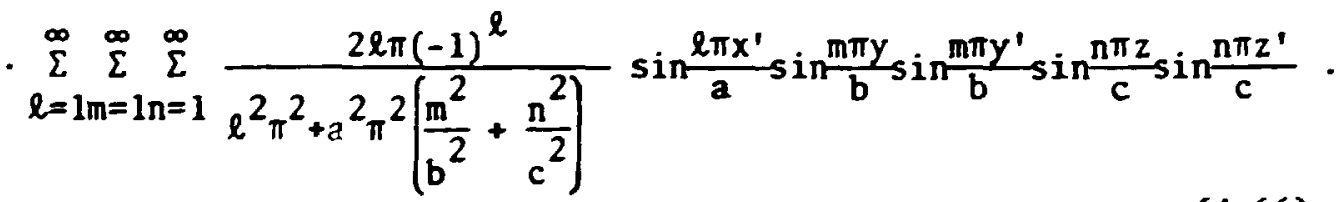

Using
$\sum_{\ell=1}^{\infty} \frac{2 \pi \ell(-1)^{\ell}}{\ell^{2} \pi^{2}+a^{2} \pi^{2}\left(\frac{m^{2}}{b^{2}}+\frac{n^{2}}{c^{2}}\right)} \sin \frac{\ell \pi x}{a}=-\frac{\sinh \pi x\left(\frac{m^{2}}{b^{2}}+\frac{n^{2}}{c^{2}}\right)^{1 / 2}}{\sinh \pi a\left(\frac{m^{2}}{b^{2}}+\frac{n^{2}}{c^{2}}\right)^{1 / 2}}$, 
and setting $\left.K_{3} \frac{\partial T_{3}}{\partial x}\right|_{x=a}=\left.K_{1} \frac{\partial T_{1}}{\partial x}\right|_{x=a}$ gives

$-\frac{4 \pi}{b c}\left(K_{1}+K_{3}\right) \sum_{m=1 n=1}^{\infty} \sum_{b^{2}}^{\infty}\left(\frac{m^{2}}{c^{2}}\right)^{2 / 2} \frac{\cosh \pi a\left(\frac{n^{2}}{b^{2}}+\frac{n^{2}}{c^{2}}\right)^{1 / 2}}{\sinh \pi a\left(\frac{n^{2}}{b^{2}}+\frac{n^{2}}{c^{2}}\right)^{1 / 2} \sin \frac{m \pi y}{b} \sin \frac{n \pi z}{c} G(m, n)}$

$=-\frac{4 \pi}{b c} K_{1} \sum_{m=1 n=1}^{\infty} \sum_{b^{2}}^{\infty}\left(\frac{m^{2}}{c^{2}}\right)^{\frac{n^{2}}{1 / 2}} \frac{\sin \frac{\pi y}{b} \sin \frac{n \pi x}{c}}{\sinh \pi a\left(\frac{m^{2}}{b^{2}}+\frac{n^{2}}{c^{2}}\right)^{1 / 2}} F(n, n)-\frac{4}{b c}$

$\cdot \sum_{m=1 n=1}^{\infty} \frac{\sinh \pi x^{\prime}\left(\frac{m^{2}}{b^{2}}+\frac{n^{2}}{c^{2}}\right)^{1 / 2}}{\sinh \pi a\left(\frac{m^{2}}{b^{2}}+\frac{n^{2}}{c^{2}}\right)^{1 / 2}} \sin \frac{m \pi y}{b} \sin \frac{m \pi y^{\prime}}{b i n} \frac{n \pi z}{c} \sin \frac{n \pi z^{\prime}}{c}$.

For termwise equality

$$
\begin{aligned}
& \pi\left(K_{1}+K_{3}\right)\left(\frac{m^{2}}{b^{2}}+\frac{n^{2}}{c^{2}}\right)^{1 / 2} \cosh \pi a\left(\frac{m^{2}}{b^{2}}+\frac{n^{2}}{c^{2}}\right)^{1 / 2} G(m, n) \\
& =\pi K_{1}\left(\frac{m^{2}}{b^{2}}+\frac{n^{2}}{c^{2}}\right)^{1 / 2} F(m, n)+\sinh \pi x^{\prime}\left(\frac{m^{2}}{b^{2}}+\frac{n^{2}}{c^{2}}\right)^{1 / 2} \sin \frac{m \pi y^{\prime}}{b} \sin \frac{n \pi z^{\prime}}{c} .
\end{aligned}
$$

This gives two equations for $F(m, n)$ and $G(m, n)$. Solving these gives 


$$
\begin{aligned}
F(m, n)= & \frac{\sin \frac{m \pi y^{\prime}}{b} \sin \frac{n \pi z^{\prime}}{c}}{\left(\frac{m^{2}}{b^{2}}+\frac{n^{2}}{c^{2}}\right)^{1 / 2}} \\
\cdot & \left\{\frac{\left(K_{1}+K_{3}\right) \cosh \pi a\left(\frac{m^{2}}{b^{2}}+\frac{n^{2}}{c^{2}}\right)^{1 / 2} \sinh \left[\pi\left(a-x^{\prime}\right)\left(\frac{m^{2}}{b^{2}}+\frac{n^{2}}{c^{2}}\right)^{1 / 2}\right]}{\left.\left(K_{1}+K_{3}\right)\left(K_{1}+K_{2}\right) \cosh ^{2}\left[\pi a\left(\frac{m^{2}}{b^{2}}+\frac{n^{2}}{c^{2}}\right)^{1 / 2}\right]-K_{1}^{2}\right]}\right. \\
& +\frac{K_{1} \sinh \pi x^{\prime}\left(\frac{m^{2}}{b^{2}}+\frac{n^{2}}{c^{2}}\right)^{1 / 2}}{\left(K_{1}+K_{3}\right)\left(K_{1}+K_{2}\right) \cosh ^{2}\left[\pi a\left(\frac{m^{2}}{b^{2}}+\frac{n^{2}}{c^{2}}\right)^{1 / 2}\right]-K_{1}{ }^{2}},
\end{aligned}
$$

and

$$
\begin{aligned}
G(m, n)= & \frac{\sin \frac{m \pi y^{\prime}}{b} \sin \frac{n \pi z^{\prime}}{c}}{\left(\frac{m^{2}}{b^{2}}+\frac{n^{2}}{c^{2}}\right)^{1 / 2}} \int \frac{\left(K_{1}+K_{2}\right) \cosh \pi a\left(\frac{m^{2}}{b^{2}}+\frac{n^{2}}{c^{2}}\right)^{1 / 2} \sinh \pi x \cdot\left(\frac{m^{2}}{b^{2}}+\frac{n^{2}}{c^{2}}\right)^{1 / 2}}{\left(K_{1}+K_{3}\right)\left(K_{1}+K_{2}\right) \cosh ^{2}\left[\pi a\left(\frac{m^{2}}{b^{2}}+\frac{n^{2}}{c^{2}}\right)^{1 / 2}\right]-K_{1}^{2}} \\
& +\frac{K_{1} \sinh \left[\pi\left(a-x^{\prime}\right)\left(\frac{m^{2}}{b^{2}}+\frac{n^{2}}{c^{2}}\right)^{1 / 2}\right]}{\left.\left(K_{1}+K_{3}\right)\left(K_{1}+K_{2}\right) \cosh ^{2}\left[\pi a\left(\frac{m^{2}}{b^{2}}+\frac{n^{2}}{c^{2}}\right)^{1 / 2}\right]-K_{1}^{2}\right)}
\end{aligned}
$$

Note that

$$
\lim _{K_{3} \rightarrow \infty} F(m, n)=\frac{\sinh \left[\pi\left(a-x^{\prime}\right)\left(\frac{m^{2}}{b^{2}}+\frac{n^{2}}{c^{2}}\right)^{1 / 2}\right]}{\pi\left(K_{1}+K_{2}\right)\left(\frac{m^{2}}{b^{2}}+\frac{n^{2}}{c^{2}}\right)^{1 / 2} \cosh \pi a\left(\frac{m^{2}}{b^{2}}+\frac{n^{2}}{c^{2}}\right)^{1 / 2}},
$$


and

$$
\lim _{K_{3} \rightarrow \infty} G(m, n)=0
$$

which gives the two-media RPP Green's function derived previously. Using $F(m, n)$ and $G(m, n)$ then gives

$$
\begin{aligned}
& T_{2}(x, y, z)=\frac{4}{\pi b c} \sum_{m=1 n=1}^{\infty} \sum_{\sum}^{\infty} \frac{\sin \frac{m \pi y^{\prime}}{b} \sin \frac{m \pi y}{b} \sin \frac{n \pi z}{c} \sin \frac{n \pi z^{\prime}}{c} \sinh \left[\pi(a+x)\left(\frac{m^{2}}{b^{2}}+\frac{n^{2}}{c^{2}}\right)^{1 / 2}\right]}{\left(\frac{m^{2}}{b^{2}}+\frac{n^{2}}{c^{2}}\right)^{1 / 2}} \frac{\sinh \left[\pi a\left(\frac{m^{2}}{b^{2}}+\frac{n^{2}}{c^{2}}\right)^{1 / 2}\right]}{[\pi} \\
& \cdot\left\{\frac{\left(K_{1}+K_{3}\right) \cosh \pi a\left(\frac{m^{2}}{b^{2}}+\frac{n^{2}}{c^{2}}\right)^{1 / 2} \sinh \left[\pi\left(a-x^{\prime}\right)\left(\frac{m^{2}}{b^{2}}+\frac{n^{2}}{c^{2}}\right)^{1 / 2}\right]}{\left(K_{1}+K_{3}\right)\left(K_{1}+K_{2}\right) \cosh ^{2}\left[\pi a\left(\frac{m^{2}}{b^{2}}+\frac{n^{2}}{c^{2}}\right)^{1 / 2}\right]-K_{1}^{2}}\right. \\
& \left.\left.+\frac{K_{1} \sinh \pi x \cdot\left(\frac{m^{2}}{b^{2}}+\frac{n^{2}}{c^{2}}\right)^{1 / 2}}{\left(K_{1}+K_{3}\right)\left(K_{1}+K_{2}\right) \cosh ^{2} \pi a\left(\frac{m^{2}}{b^{2}}+\frac{n^{2}}{c^{2}}\right)^{1 / 2}-k_{1}^{2}}\right)\right\}
\end{aligned}
$$

for $-a \leq x \leq 0,0 \leq y \leq b, 0 \leq z \leq c$, 


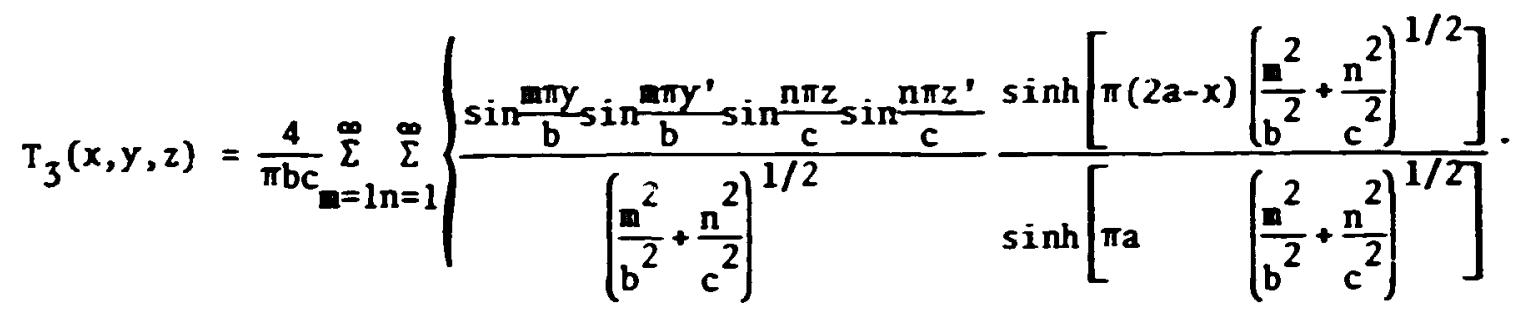

$$
\begin{aligned}
& \left\{\frac{\left(K_{1}+K_{3}\right) \cosh \pi a\left(\frac{m^{2}}{b^{2}}+\frac{n^{2}}{c^{2}}\right)^{1 / 2} \sinh \pi x \cdot\left(\frac{m^{2}}{b^{2}}+\frac{n^{2}}{c^{2}}\right)^{1 / 2}}{\left(K_{1}+K_{3}\right)\left(K_{1}+K_{2}\right) \cosh ^{2}\left[\pi a\left(\frac{m^{2}}{b^{2}}+\frac{n^{2}}{c^{2}}\right)^{1 / 2}\right]-K_{1}^{2}}\right. \\
& \left.\left.+\frac{k_{1} \sinh \left[\pi\left(a-x^{\prime}\right)\left(\frac{m^{2}}{b^{2}}+\frac{n^{2}}{c^{2}}\right)^{1 / 2}\right]}{\left(k_{1}+K_{3}\right)\left(K_{1}+k_{2}\right) \cosh ^{2}\left[\pi a\left(\frac{m^{2}}{b^{2}}+\frac{n^{2}}{c^{2}}\right)^{1 / 2}\right]-k_{1}^{2}}\right)\right\},
\end{aligned}
$$

for $a \leq x \leq 2 \bar{a}, 0 \leq y \leq b, 0 \leq z \leq c$,

and

$$
\begin{aligned}
T_{1}(x, y, z)= & T_{2}(-x, y, z)+T_{3}(2 a-x, y, z)+\frac{8}{K_{1} \pi^{2} a b c} \\
& \cdot \sum_{\ell=1 m=1 n=1}^{\infty} \sum_{\frac{l^{2}}{a^{2}}+\frac{m^{2}}{b^{2}}+\frac{n^{2}}{c^{2}}}^{\infty} \operatorname{in} \frac{l \pi x}{a} \sin \frac{\ell \pi x^{\prime}}{a} \sin \frac{m \pi y}{b} \sin \frac{m \pi y^{\prime}}{b} \sin \frac{n \pi z}{c} \sin \frac{n \pi z^{\prime}}{c},
\end{aligned}
$$

for $0 \leq x \leq a, 0 \leq y \leq b, 0 \leq z \leq c$. 


\section{THREE-AEDIA RPP WITH SINUSOIDAL}

INTERNAL SOURCE

Problem 5: To calculate the temperature distribution in a threemedia, rectangular parallelepiped with no contact resistance between media and with a fixed, uniform temperature on all external boundaries (Figure 5).

Assume :

1. $k_{1}$ is the thermal conductivity for $0 \leq x \leq a, 0 \leq y \leq b$, $0 \leq 2 \leq c$

2. $K_{2}$ is the thermal conductivity for $-a \leq x \leq 0,0 \leq y \leq b$, $0 \leq z \leq c$

3. $K_{3}$ is the thermal conductivity for $a \leq x \leq 2 a, 0 \leq y \leq b$, $0 \leq z \leq c$

4. Steady state

5. $S=S_{0} \sin \pi x / a \sin \pi y / b \sin \pi z / c$ for $0 \leq x \leq a, 0 \leq y \leq b$, $0 \leq z \leq c$

The analytical solution for this problem can be cbtained using the previously generated Green's function.

$T(x, y, z)=\iiint_{V} T_{g^{\prime}}\left(x, y, z, x^{\prime}, y^{\prime}, z^{\prime}\right) S\left(x^{\prime}, y^{\prime}, z^{\prime}\right) d x^{\prime} d y^{\prime} d z^{\prime}$

Using

$$
\int_{0}^{b} \sin \frac{\pi y}{b} \sin \frac{m \pi y}{b} d y= \begin{cases}b / 2, & m=1, \\ 0, & m \neq 1,\end{cases}
$$

and 


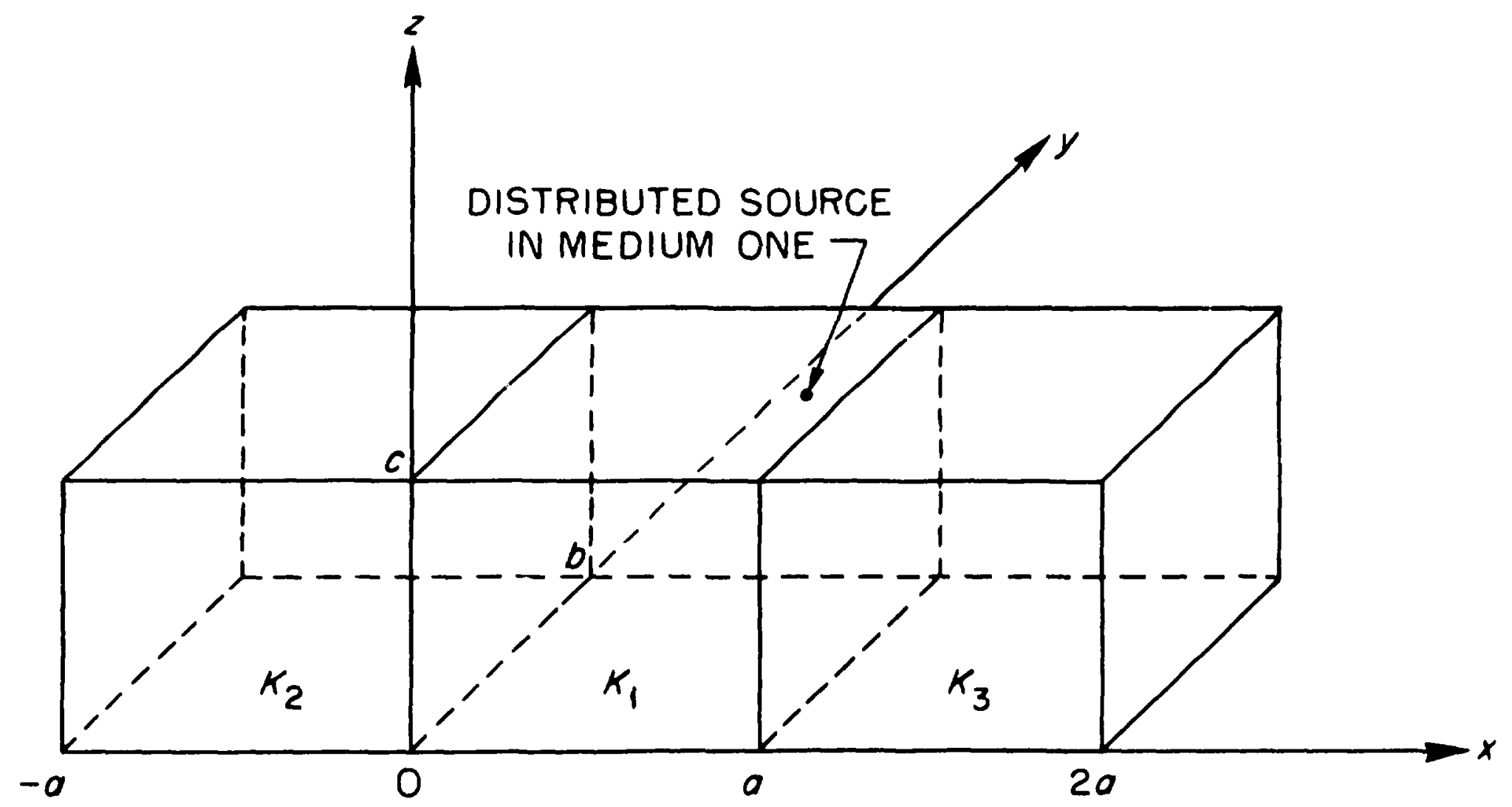

Figure 5. Three-media RPP with distributed source in medium one. 
$\int_{0}^{a} \sinh \left[\pi(a-x)\left(\frac{m^{2}}{b^{2}}+\frac{n^{2}}{c^{2}}\right)^{1 / 2}\right] \sin \frac{\pi x}{a} d x=\frac{a \sinh \pi a\left(\frac{m^{2}}{b^{2}}+\frac{n^{2}}{c^{2}}\right)^{1 / 2}}{\pi+a^{2} \pi\left(\frac{m^{2}}{b^{2}}+\frac{n^{2}}{c^{2}}\right)^{2}}$,

gives

$$
\begin{aligned}
T_{2}(x, y, z)= & \frac{s_{0} a}{\pi^{2}} \frac{1}{\left(\frac{1}{b^{2}}+\frac{1}{c^{2}}\right)^{1 / 2}} \cdot \frac{1}{1+a^{2}\left(\frac{1}{b^{2}}+\frac{1}{c^{2}}\right)} \\
& \frac{\left(K_{1}+K_{3}\right) \cosh \left[\pi a\left(\frac{1}{b^{2}}+\frac{1}{c^{2}}\right)^{1 / 2}\right]+K_{1}}{\left(K_{1}+K_{3}\right)\left(K_{1}+K_{2}\right) \cosh ^{2}\left[\pi a\left(\frac{1}{b^{2}}+\frac{1}{c^{2}}\right)^{1 / 2}\right]-K_{1}^{2}} \\
& \cdot \sinh \left[\pi(a+x)\left(\frac{1}{b^{2}}+\frac{1}{c^{2}}\right)^{1 / 2}\right] \sin \frac{\pi y}{b i n} \frac{\pi 2}{c},
\end{aligned}
$$

$$
\begin{aligned}
& \text { for }-a \leq x \leq 0,0 \leq y \leq b, 0 \leq z \leq c \text {, } \\
& T_{3}(x, y, z)=\frac{S_{0} a}{\pi^{2}}\left(\frac{1}{\left(\frac{1}{b^{2}}+\frac{1}{c^{2}}\right)^{1 / 2}} \cdot \frac{1}{1+a^{2}\left(\frac{1}{b^{2}}+\frac{1}{c^{2}}\right)}\right. \\
& \frac{\left(K_{1}+K_{3}\right) \cosh \left[\pi a\left(\frac{1}{b^{2}}+\frac{1}{c}\right)^{1 / 2}\right]+K_{1}}{\left(K_{1}+K_{3}\right)\left(K_{1}+K_{2}\right) \cosh ^{2}\left[\pi a\left(\frac{1}{b^{2}}+\frac{1}{c^{2}}\right)^{1 / 2}\right]-K_{1}^{2}}
\end{aligned}
$$




$$
\text { - } \left.\sinh \left[\pi(2 a-x)\left(\frac{1}{b^{2}}+\frac{1}{c^{2}}\right)^{1 / 2}\right] \sin \frac{\pi y}{b} \sin \frac{\pi z}{c}\right) \text {, }
$$

for $a \leq x \leq 2 a, 0 \leq y \leq b, 0 \leq z \leq c$,

and

$$
\begin{aligned}
T_{1}(x, y, z)= & T_{2}(-x, y, z)+T_{3}(2 a-x, y, z) \\
& +\frac{S_{0}}{\pi^{2} K_{1}} \frac{1}{\frac{1}{a^{2}}+\frac{1}{b^{2}}+\frac{1}{c^{2}}} \sin \frac{\pi x}{a} \sin \frac{\pi y}{b} \sin \frac{\pi z}{c},
\end{aligned}
$$

for $0 \leq x \leq a, 0 \leq y \leq b, 0 \leq z \leq c$.

ihis solution is verified in Appendix A. The parameters for the problem are:

1. $K_{1}=.1667 \mathrm{cal} / \mathrm{sec} \mathrm{cm}^{\circ} \mathrm{C}$

2. $\mathrm{K}_{2}=.3333 \mathrm{cal} / \mathrm{sec} \mathrm{cm}^{\circ} \mathrm{C}$

3. $K_{3}=.3333 \mathrm{cal} / \mathrm{sec} \mathrm{cm}^{\circ} \mathrm{C}$

4. $a=1 \mathrm{~cm}$

5. $b=1 \mathrm{~cm}$

6. $c=1 \mathrm{~cm}$

7. $S_{0}=238.8(\pi / 2)^{3} \mathrm{cal} / \mathrm{sec} \mathrm{cm}^{3}$, with an external surface temperature of $80^{\circ} \mathrm{C}$.

The Monte Carlo solution was performed using the adjoint formulation. A scaling factor of 10 was used. Adjoint calculations were made for the temperature at four points along the $y=.5, z=.5$ 
centerline. Each Monte Carlo calculation represents 4.5 min of IBM 360/91 CPU tine. The results are shown in Table 7.

VIII. TWO-AEDIA RCC GREEN'S FUNCTIONS

The next series of problens requires analytical solutions for twomedia right circular cylinders. The first step in generating the solutions to these problems is to derive an appropriate Green's function (Figure 6).

From Carslaw and Jaeger, 22 the one-medium Green's function for a right circular cylinder (RCC) $0 \leq z \leq \ell, 0 \leq \theta \leq \pi, 0 \leq r \leq$ a with zero surface temperature and a unit instantaneous point source at $\left(z^{\prime}, \theta^{\prime}, r^{\prime}, t=0\right)$ is

$$
\begin{aligned}
T= & \frac{2}{\pi a^{2} l^{m=1}} \sum_{1}^{\infty}\left[e^{-K m^{2} \pi^{2} \pm ; l^{2}} \sin \frac{m \pi z}{l} \sin \frac{m \pi z^{\prime}}{l}\right. \\
& \left.\cdot \sum_{n=-\infty}^{\infty} \cos n\left(\theta-\theta^{\prime}\right) \Sigma e^{-K \alpha^{2} t} \frac{J_{n}(\alpha r) J_{n}\left(\alpha r^{\prime}\right)}{\left[J_{n}^{\prime}(\alpha a)\right]^{2}}\right],
\end{aligned}
$$

where $\alpha$ are positive roots of

$$
J_{n}(\alpha a)=0 \text {. }
$$

For a source with symetry about the 2 axis, the integration over $\theta$ can be performed using 
TABLE 7

TEMPERATURE COMPARISONS FOR A THREE-MEDIA RPP WITH

A SINUSOIDAL INTERNAL SOURCE

\begin{tabular}{|c|c|c|c|}
\hline $\begin{array}{c}x \\
(\mathrm{~cm}) \\
\end{array}$ & $\begin{array}{c}\text { Analytic } \\
\text { Temperature } \\
{ }^{\circ} \mathrm{C} \\
\end{array}$ & $\begin{array}{l}\text { Monte Carlo } \\
\text { Temperature } \\
{ }^{\circ} \mathrm{C}\end{array}$ & $\begin{array}{l}\text { Estimated } \\
\text { Standard } \\
\text { Deviation } \\
\mathrm{C}^{\circ}\end{array}$ \\
\hline-.7 & 81.849 & 82.6 & .16 \\
\hline-.5 & 84.89 & 86.7 & .32 \\
\hline .1 & 166.9 & 176.7 & 1.93 \\
\hline .5 & 276.9 & 290.6 & 2.30 \\
\hline
\end{tabular}




\section{RING SOURCE IN MEDIUM ONE}

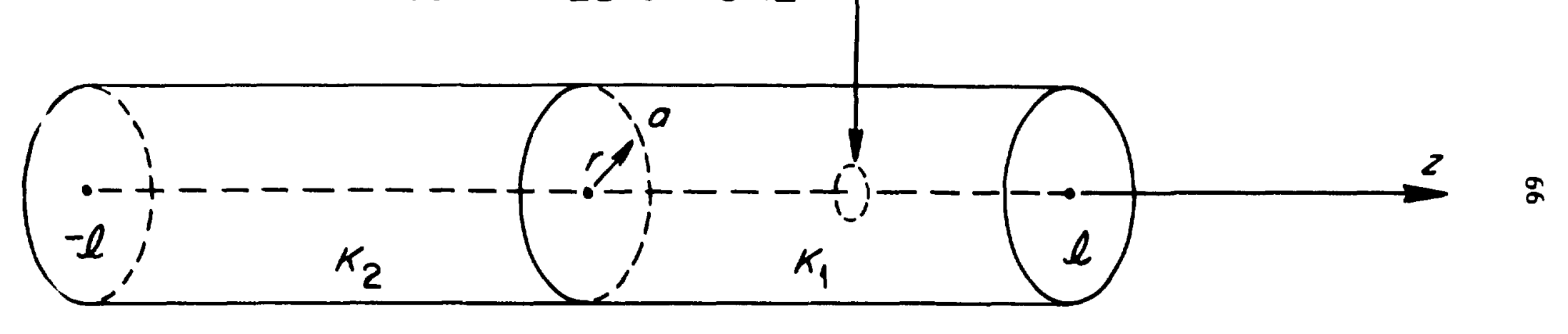

Figure 6. Two-media RCC with a ring source in medium one. 
$\int_{0}^{2 \pi} \cos n\left(\theta-\theta^{\prime}\right) d \theta= \begin{cases}2 \pi, & n=0, \\ 0, & n \neq 0,\end{cases}$

to give

$T=\frac{4}{a^{2} l} \sum_{m=1}^{\infty} e^{-K m^{2} \pi^{2} t / l^{2}} \sin \frac{m \pi z}{\ell} \sin \frac{m \pi z^{\prime}}{\ell} \sum_{\alpha} e^{-K \alpha t} \frac{J_{0}(\alpha r) J_{0}\left(\alpha r^{\prime}\right)}{\left[J_{0}^{2}(\alpha a)\right]^{2}}$,

where $\alpha$ are positive roots of $J_{0}(\alpha a)=0$, or, since $J_{0^{\prime}}(x)=-J_{1}(x)$

$T=\frac{4}{a^{2} \ell} \sum_{m=1}^{\infty} e^{-K_{m}^{2} \pi^{2} t / \ell^{2}} \sin \frac{m \pi z}{\ell} \sin \frac{m \pi z^{\prime}}{\ell} \sum_{n=1}^{\infty} e^{-K \alpha_{n} t} \frac{J_{0}\left(\alpha_{n} r\right) J_{0}\left(\alpha_{n} r^{\prime}\right)}{J_{1}{ }^{2}\left(\alpha_{n} a\right)}$,

where $\alpha_{n}$ are positive roots of $J_{0}\left(a_{n} a\right)=0$.

For steady state the solution is given by $\lim _{t \rightarrow \infty} \int_{0}^{t} T(r, z, t-\tau) d \tau$. Using

$$
\int_{0}^{t} e^{-k\left(m^{2} \pi^{2} / l^{2}+\alpha_{n}^{2}\right)(t-\tau)} d \tau=\frac{1}{k\left(m^{2} \pi^{2} / l^{2}+\alpha_{n}^{2}\right)} 1-e^{-k\left(m^{2} \pi^{2} / l^{2}+\alpha_{n}^{2}\right) t} \text {, }
$$

taking $\lim _{t \rightarrow \infty}$ gives $\frac{1}{k\left(\frac{m^{2} \pi^{2}}{2}+\alpha_{n}^{2}\right)}$

or, for a steady-state source symmetric about the $z$ axis

$T_{g}=\frac{4}{a^{2} l} \sum_{m=1}^{\infty} \sin \frac{m \pi z}{\ell} \sin \frac{m \pi z^{\prime}}{\ell} \sum_{n=1}^{\infty} \frac{1}{k\left(\frac{m^{2} \pi^{2}}{2}+\alpha_{n}^{2}\right)} \frac{J_{0}\left(n_{n} r\right) J_{0}\left(a_{n} r^{\prime}\right)}{J_{1}{ }^{2}\left(a_{n} a\right)}$ 
If the RCC face is maintained at $f(r)$, other faces at zero, the temperature is given by

$$
T=\left.K \int_{0}^{a} r^{\prime} f\left(r^{\prime}\right) \frac{\partial T_{g}}{\partial z^{\prime}}\right|_{z^{\prime}=0} d r^{\prime}
$$

This gives

$$
T=\frac{4 \pi}{a^{2} l^{2}} \sum_{n=1}^{\infty} m \sin \frac{m \pi z}{\ell} \sum_{n=1}^{\infty} \frac{1}{\left[\frac{m^{2} \pi^{2}}{l^{2}}+\alpha_{n}^{2}\right]} \frac{J_{0}\left(a_{n} r\right)}{J_{1}^{2}\left(a_{n} a\right)} \int_{0}^{a} r^{\prime} f\left(r^{\prime}\right) J_{0}\left(a_{n} r^{\prime}\right) d r^{\prime}
$$

Substituting

$$
\sum_{m=1}^{\infty} \frac{2 \pi m}{\ell^{2}} \frac{1}{\left[\frac{m^{2} \pi^{2}}{l^{2}}+\alpha_{n}^{2}\right]} \sin \frac{m \pi z}{\ell}=\frac{\sinh \alpha_{n}(\ell-z)}{\sinh \alpha_{n} l}
$$

into Equation (4-91) gives

$T=\frac{2}{a^{2}} \sum_{n=1}^{\infty} \frac{J_{0}\left(a_{n} r\right)}{J_{1}{ }^{2}\left(a_{n} a\right)} \frac{\sinh \alpha_{n}(l-z)}{\sinh \alpha_{n} l} \int_{0}^{a} r^{\prime} f\left(r^{\prime}\right) J_{0}\left(a_{n} r^{\prime}\right) d r^{\prime}$.

To obtain a Green's function for a composite two-media region, first assume an RCC $-\ell \leq z \leq 0,0 \leq r \leq a, K=K_{2}$ and $f(r)$ at $z=0$, with the other faces at zero, then

$$
T_{2}=\frac{2}{3^{2}} \sum_{n=1}^{\infty} \frac{J_{0}\left(\alpha_{n} r\right)}{J_{1}{ }^{2}\left(\alpha_{n} a\right)} \frac{\sinh a_{n}(\ell+z)}{\sinh \alpha_{n} l} \int_{0}^{a} r^{\prime} f\left(r^{\prime}\right) J_{0}\left(c_{n} r^{\prime}\right) d r^{\prime}
$$


Now assume an RCC $0 \leq z \leq \ell, 0 \leq r \leq a, K=K_{1}$, and $f(r)$ at $z=0$ with the other faces at zero and a symetric ring source at $Z^{\prime}, I^{\prime}$, then

$$
\begin{aligned}
T_{1}= & T_{2}(r,-z)+\frac{4}{a^{2} \ell} \sum_{m=1}^{\infty}\left[\sin \frac{m \pi z}{\ell} \sin \frac{m \pi z^{\prime}}{l}\right. \\
& \left.\sum_{n=1}^{\infty} \frac{1}{k_{1}\left(\frac{m^{2} \pi^{2}}{l^{2}}+a_{n}{ }^{2}\right)} \frac{J_{0}\left(a_{n} r\right) J_{Q}\left(a_{n} r^{\prime}\right)}{J_{1}{ }^{2}\left(a_{n} a\right)}\right] .
\end{aligned}
$$

For continuity of heat flow at $z=0$

$$
\left.K_{2} \frac{\partial T_{2}}{\partial z}\right|_{z=0}=\left.K_{1} \frac{\partial T_{1}}{\partial z}\right|_{z=0}
$$

From Equation (4-94)

$\left.\frac{\partial T_{2}}{\partial z}\right|_{z=0}=\frac{2}{a^{2}} \sum_{n=1}^{\infty} \frac{J_{0}\left(a_{n} r\right)}{J_{1}{ }^{2}\left(a_{n} a\right)} \frac{a_{n} \cosh a_{n} \ell}{\sinh \alpha_{n} l} \int_{0}^{a} r^{\prime} f\left(r^{\prime}\right) J_{0}\left(a_{n} r^{\prime}\right) d r^{\prime}$.

From Equation (4-95)

$\left.\frac{\partial T}{\partial z}\right|_{z=0}=-\left.\frac{\partial T_{2}}{\partial z}\right|_{z=0}+\frac{4 \pi}{a^{2} \ell^{2}} \sum_{m=1}^{\infty} m \sin \frac{m \pi z^{\prime}}{\ell} \sum_{n=1}^{\infty} \frac{1}{K_{1}\left(\frac{m^{2} \pi^{2}+\alpha_{n}}{\ell^{2}}\right)} \frac{J_{Q}\left(\alpha_{n} r\right) J_{Q}\left(\alpha_{n} r^{\prime}\right)}{J_{1}^{2}\left(a_{n} a\right)}$.

Using

$$
\sum_{m=1}^{\infty} \frac{2 \pi m}{\ell^{2}} \frac{1}{\left[\frac{m^{2} \pi^{2}}{\ell^{2}}+\alpha_{n}^{2}\right]} \sin \frac{m \pi z}{\ell}=\frac{\sinh (\ell-z) \alpha_{n}}{\sinh \alpha_{n} l}
$$


and setting

$$
\left.K_{2} \frac{\partial T_{2}}{\partial z}\right|_{z=0}=\left.K_{1} \frac{\partial T_{1}}{\partial z}\right|_{z=0}
$$

gives

$$
\begin{aligned}
& \frac{2}{a^{2}}\left(K_{1}+K_{2}\right) \sum_{n=1}^{\infty} \frac{J_{0}\left(a_{n} r\right)}{J_{1}{ }^{2}\left(a_{n} a\right)} a_{n} \frac{\cosh a_{n} l}{\sinh a_{n} l} \int_{0}^{a} r^{\prime} f\left(r^{\prime}\right) J_{0}\left(a_{n} r^{\prime \prime}\right) d r^{\prime} \\
& =\frac{2}{a^{2}} \sum_{n=1}^{\infty} \frac{J_{0}\left(a_{n} r\right) J_{0}\left(a_{n} r^{\prime}\right)}{J_{1}{ }^{2}\left(a_{n} a\right)} \frac{\sinh \left(\ell-z^{\prime}\right) a_{n}}{\operatorname{sinhl} l a_{n}} .
\end{aligned}
$$

Assuming termwise equality gives

$$
\int_{0}^{a} r^{\prime} f\left(r^{\prime}\right) J_{0}\left(\alpha_{n} r^{\prime}\right) d r^{\prime}=\frac{1}{K_{1}+K_{2}} \frac{\sinh \alpha_{n}\left(l-z^{\prime}\right)}{\alpha_{n} \cosh \sigma_{n} l} J_{0}\left(\alpha_{n} r^{\prime}\right)
$$

Substituting Equation (4-102) into Equations (4-94) and (4-95) gives the solution as

$$
T_{2}\left(r, z, r^{\prime}, z^{\prime}\right)=\frac{2}{a^{2}\left(K_{1}+K_{2}\right)} \sum_{n=1}^{\infty} \frac{J_{0}\left(a_{n} r\right) J_{0}\left(a_{n} r^{\prime}\right)}{\alpha_{n} \sinh \alpha_{n} l} \frac{\sinh _{n}(\ell+z) \sinh \alpha_{n}\left(\ell-z^{\prime}\right)}{\cosh a_{n} l},
$$

and

$$
\begin{aligned}
T_{1}\left(r, z, r^{\prime}, z^{\prime}\right)= & T_{2}\left(r,-z, r^{\prime}, z^{\prime}\right) \\
& +\frac{4}{a^{2} \ell} \sum_{m=1}^{\infty} \sin \frac{m \pi z}{\ell} \sin \frac{m \pi z^{\prime}}{\ell} \sum_{n=1}^{\infty} \frac{1}{k_{1}\left(\frac{m^{2} \pi^{2}}{l^{2}}+\alpha_{n}^{2}\right)} \frac{J_{0}\left(\alpha_{n} r\right) J_{0}\left(\alpha_{n} r^{\prime}\right)}{J_{1}{ }^{2}\left(\alpha_{n^{2}}\right)} .
\end{aligned}
$$


With the form of the Green's function solution $T_{g}\left(r, z, r^{\prime}, z^{\prime}\right)$, if $S(r, z, \theta)$ is any arbitrary source in medium one symmetric about $z$, i.e., $S(r, z, \theta)=S(r, z)$, then the temperature is given by

$$
T=\iint_{z} r_{r^{\prime}} S\left(r^{\prime}, z^{\prime}\right) T_{g}\left(r, z, r^{\prime}, z^{\prime}\right) r^{\prime} d r^{\prime} d z^{\prime}
$$

\section{TWO-MEDIA RCC WITH FUNCTIONAL INTERNAL SOURCE}

Problem 6: To calculate the temperature distribution in a twomedia, right circular cylinder (Figure 7).

Assume :

1. $K_{1}$ is the thermal conductivity for $0 \leq s_{2} \leq \ell, 0 \leq r \leq a$

2. $K_{2}$ is the thermal conductivity for $-\ell \leq z \leq 0,0 \leq r \leq a$

3. Steady state

4. No contact resistance between media

5. All external surface temperatures are zero

6. $S=S_{0} J_{0}\left(r a_{1}\right) \sin \pi z / \ell, 0 \leq r \leq a, 0 \leq z \leq \ell$.

The analytical solution for this problem can be obtained using the previously generated Green's function.

Using

$$
\begin{aligned}
& \int_{0}^{a} r J_{0}\left(\alpha_{n} r\right) J_{0}\left(\alpha_{m} r\right) d r=\left\{\begin{array}{lll}
\frac{a^{2}}{2} & J_{1}\left(\alpha_{n} a\right)^{2}, & n=m, \\
0 & , & n \neq m,
\end{array}\right. \\
& \int_{0}^{\ell} \sin \frac{m \pi z}{\ell} \sin \frac{\pi z}{\ell} d z= \begin{cases}\ell / 2 & m=1, \\
0, & m \neq 1,\end{cases}
\end{aligned}
$$




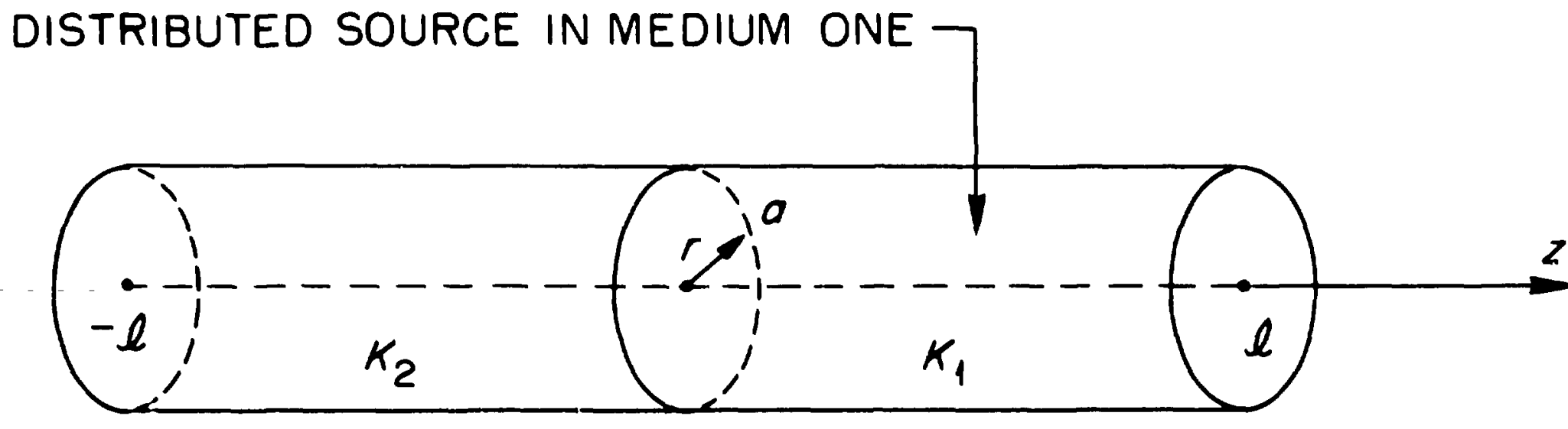

Figure 7. Two-media RCC with a distributed source in medium one. 
and

$$
\int_{0}^{\ell} \sinh \alpha_{n}(\ell-z) \sin \pi z d z=\frac{\pi \ell}{\pi^{2}+\ell^{2} \alpha_{n}^{2}} \sinh \alpha_{n^{2}}
$$

gives

$$
\mathrm{T}_{2}(\mathrm{r}, z)=\frac{\mathrm{S}_{0} \pi l}{\pi^{2}+l^{2} \alpha_{1}^{2}} \frac{\mathrm{J}_{0}\left(\alpha_{1} r\right)}{\alpha_{1}\left(K_{1}+K_{2}\right)} \frac{\sinh \alpha_{1}(l+z)}{\cosh \alpha_{1} l}
$$

for $0 \leq r \leq a,-\ell \leq z \leq c$,

and

$$
T_{1}(r, z)=T_{2}(r,-z)+\frac{s_{0}}{K_{1}\left(\alpha_{1}^{2}+\frac{\pi^{2}}{\ell^{2}}\right)} J_{0}\left(\alpha_{1} r\right) \sin \frac{\pi z}{\ell},
$$

for $0 \leq r \leq a, 0 \leq z \leq \ell$.

This solution is verified in Appendix $A$.

The parameters for the problem are

1. $\mathrm{K}_{1}=.1667 \mathrm{cal} / \mathrm{sec} \mathrm{cm}^{\circ} \mathrm{C}$

2. $\mathrm{K}_{2}=.3333 \mathrm{cal} / \mathrm{sec} \mathrm{cm}^{\circ} \mathrm{C}$

3. $a=.5 \mathrm{~cm}$

4. $\ell=1 \mathrm{~cm}$

5. $\mathrm{S}=238.8(4.632259) \mathrm{J}_{0}\left(\mathrm{r} \alpha_{1}\right) \sin \pi \mathrm{z} \mathrm{cal} / \mathrm{sec} \mathrm{cm}^{2}\left\{\begin{array}{l}0 \leq \mathrm{r} \leq .5 \\ 0 \leq z \leq 1\end{array}\right.$

with an external surface temperature of $80^{\circ} \mathrm{C}$. The constant 4.632259 is the normalization factor for a unit source. 
The Monte Carlo solution was performed using the adjoint formulation and a scaling factor of 10. Adjoint calculations were made for the temperature at four points along the $r=0$ centerline. Each Monte Carlo calculation required 4.5 min of IBM $360 / 91$ CPU time. The results are shown in Table 8 .

The use of the scale factor to allow the transport equation to more closely approximate the diffusion equation was studied for this problem. Calculations were made using the DOT $^{27}$ discrete ordinates radiation transport code. These results are shown in Table 9. (The DOT diffusion theory calculations include an extrapolation distance correction to approximate transport theory.) As (ail be seen, the transport equation approximation can be made as close as desired by increasing th: scale factor used in the calculations. However, as the scale factor is increased, the time involved in performing the calculation increases also. For example, a calculation of the temperature at $x=-.7 \mathrm{~cm}$ was calculated by Monte Carlo using a scale factor of 40 . The results obtained were $81.44^{\circ} \mathrm{C}$ with an estimated standard deviation of $.38 \mathrm{C}^{\circ}$. This run required $4.5 \mathrm{~min}$ of IBM $360 / 91$ CPU time.

\section{TWO-MEDIA RCC WITH UNIFORM INTERNAL SOURCE}

Problem 7: To calculate the temperature distribution in a twomedia, right circular cylinder as in the previous problem except with a source distribution of 
TABLE 8

TEMPERATURE COMIPARISONS FOR A TWO-MEDIA RCC WITH A FUNCTIONAL INTERNAL SOURCE

\begin{tabular}{|c|c|c|c|}
\hline $\begin{array}{c}x \\
(\mathrm{~cm}) \\
\end{array}$ & $\begin{array}{l}\text { Analytic } \\
\text { Temperature } \\
{ }^{\circ} \mathrm{C}\end{array}$ & $\begin{array}{l}\text { Monte Carlo } \\
\text { Temperature } \\
{ }^{\circ} \mathrm{C} \\
\end{array}$ & $\begin{array}{c}\text { Estimated } \\
\text { Standard } \\
\text { Deviation } \\
\mathrm{C}^{\circ} \\
\end{array}$ \\
\hline-.7 & 81.426 & 82.69 & .21 \\
\hline-.5 & 83.921 & 86.19 & .35 \\
\hline .1 & 169.209 & 180.0 & 2.16 \\
\hline .5 & 285.031 & 301.9 & 2.63 \\
\hline
\end{tabular}


TABLE 9

TEMPERATURE CO:'PARISONS FOR DOT RUNS WITH DIFFERENT SCALING FACTORS

\begin{tabular}{|c|c|c|c|c|c|c|}
\hline $\begin{array}{c}\text { Scale Factor }^{2} \\
\text { (cm) }\end{array}$ & $\begin{array}{c}\text { Analytic } \\
{ }^{\circ} \mathrm{C}\end{array}$ & $\begin{array}{c}\text { DOT } \\
\text { Diffusion } \\
1000 \\
{ }^{\circ} \mathrm{C} \\
\end{array}$ & $\begin{array}{c}\text { DOT } \\
\text { Diffusion } \\
100 \\
{ }^{\circ} \mathrm{C} \\
\end{array}$ & $\begin{array}{c}\text { DOT } \\
\text { Transport } \\
40 \\
{ }^{\circ} \mathrm{C} \\
\end{array}$ & $\begin{array}{c}\text { DOT } \\
\text { Transport } \\
20 \\
{ }^{\circ} \mathrm{C} \\
\end{array}$ & $\begin{array}{c}\text { DOT } \\
\text { Transport } \\
10 \\
{ }^{\circ} \mathrm{C} \\
\end{array}$ \\
\hline-.9 & 80.356 & 80.361 & 80.396 & 80.418 & 80.554 & 80.857 \\
\hline-.8 & 80.798 & 80.805 & 80.860 & 80.876 & 81.089 & 81.543 \\
\hline-.7 & 81.426 & 81.438 & 81.516 & 81.545 & 81.855 & 82.500 \\
\hline-.6 & 82.388 & 82.412 & 82.531 & 82.579 & 83.009 & 83.916 \\
\hline-.5 & 83.92 & 83.94 & 84.11 & 84.20 & 84.82 & 85.99 \\
\hline-.4 & 86.38 & 86.40 & 86.62 & 86.78 & 87.62 & 89.19 \\
\hline-.3 & 90.34 & 90.36 & 90.65 & 90.94 & 92.01 & 94.02 \\
\hline-.2 & 96.72 & 96.76 & 97.12 & 97.60 & 98.96 & 101.37 \\
\hline-.1 & 107.0 & 107.0 & 107.5 & 108.2 & 109.9 & 113.0 \\
\hline 0.0 & 123.7 & 123.9 & 124.4 & 125.8 & 127.5 & 131.8 \\
\hline .1 & 169.2 & 168.8 & 169.6 & 171.7 & 174.6 & 180.3 \\
\hline .2 & 214.9 & 214.4 & 215.4 & 218.3 & 221.1 & 228.3 \\
\hline .3 & 253.1 & 252.4 & 253.6 & 257.2 & 260.3 & 268.4 \\
\hline .4 & 277.7 & 277.0 & 278.2 & 282.5 & 285.6 & 294.4 \\
\hline .5 & 285.0 & 284.4 & 285.6 & 289.9 & 293.2 & 302.3 \\
\hline .6 & 273.7 & 273.0 & 274.4 & 278.4 & 281.5 & 290.9 \\
\hline .7 & 244.1 & 243.6 & 245.0 & 248.6 & 251.9 & 260.8 \\
\hline .8 & 198.9 & 198.7 & 200.1 & 202.7 & 206.6 & 215.4 \\
\hline .9 & 142.6 & 142.6 & 144.0 & 146.1 & 150.9 & 159.5 \\
\hline
\end{tabular}




$$
S(x, y, z)=S_{0},
$$

for $0 \leq r \leq a, 0 \leq z \leq \ell$

The analytical solution is obtained using the two-media, right circular cylinder Green's function.

Using the following integrals:

$$
\int_{0}^{a} r J_{0}\left(a_{n} r\right) d r=\frac{a}{a_{n}} J_{1}\left(a_{n} a\right),
$$

and

$$
\int_{0}^{l} \operatorname{sinha}_{n}(\ell-z) d z=\frac{\cosh a_{n} \ell-1}{a_{n}},
$$

gives

$T_{2}(r, z)=\frac{2 S_{0}}{a\left(K_{1}+K_{2}\right)} \sum_{n=1}^{\infty} \frac{\cosh a_{n} l-1}{\left.a_{n}^{3} J_{1}{ }^{\prime} c_{n} a\right)} \quad \frac{J_{0}\left(\alpha_{n} r\right) \sinh a_{n}(l+z)}{\sinh \alpha_{n} l \cosh \alpha_{n} l}$,

for $0 \leq \mathrm{r} \leq \mathrm{a},-\ell \leq \mathrm{z} \leq 0$,

and

$$
T_{1}(r, z)=T_{2}(r,-z)+\frac{8 S_{0}}{a \pi} \sum_{m \text { odd }}^{\infty} \frac{1}{m} \sin \frac{m \pi z}{l} \sum_{n=1}^{\infty} \frac{1}{K_{1} \alpha_{n}\left(\frac{m^{2} \pi^{2}}{l^{2}} \alpha_{n}^{2}\right)} \frac{J_{0}\left(\alpha_{n} r\right)}{J_{1}\left(\alpha_{n} a\right)},
$$

for $0 \leq \mathrm{I} \leq \mathrm{a}, 0 \leq z \leq \ell$.

This solution is verified in Appendix $A$. 
For a unit source, $S_{0}=1 / \pi a^{2} \ell$.

This solution still requires the evaluation of an infinite series to obtain numerical results. The series is absolutely convergent, however, and converges very quickly. A source of

$$
S_{0}=\frac{238.8}{\pi a^{2} l} \mathrm{cal} / \mathrm{sec},
$$

with an external bondary temperature of $80^{\circ} \mathrm{C}$ was used for the numerical calculations.

The Monte Carlo solution was performed using the adjoint formulation and a scaling factor of 10 . Adjoint calculations were made at four points along the $\mathbf{r}=0$ axis. Each Monte Carlo calculation required 4.5 min of IBM $360 / 91$ CPU time. The results of these calculations are shown in Table 10.

\section{TWO-MEDIA RCC WITH GIVEN SURFACE TEMPERATURE DISTRIBUTION}

Problem 8: To calculate the temperature distribution in a twomedia right circular cylinder as in the previous problem except:

1. No internal sources

2. $T(z=\ell)=J_{0}(\alpha, r)$.

The analytical solution is obtained using the two-media, RCC Green's function. Denoting the Green's function solution by $T_{g}\left(r, z, r^{\prime}, z^{\prime}\right)$ for a ring source at $\left(r^{\prime}, z^{\prime}\right)$, the temperature distribution due to a surface temperature $\phi(r, z)$ is given by 
TABLE 10

TEMPERATURE COMPARISONS FOR A TWO-MEDIA RCC WITH A UNIFORM INTERNAL SOURCE

\begin{tabular}{|c|c|c|c|}
\hline $\begin{array}{c}2 \\
(\mathrm{~cm}) \\
\end{array}$ & $\begin{array}{c}\text { Analytic } \\
\text { Temperature } \\
{ }^{\circ} \mathrm{C}\end{array}$ & $\begin{array}{l}\text { Monte Carlo } \\
\text { Temperature } \\
{ }^{\circ} \mathrm{C}\end{array}$ & $\begin{array}{l}\text { Estimated } \\
\text { Standard } \\
\text { Deviation } \\
\text { C }^{\circ}\end{array}$ \\
\hline-.7 & 80.716 & 80.94 & .25 \\
\hline-.5 & 81.849 & 82.86 & .51 \\
\hline .1 & 114.826 & 117.3 & 3.17 \\
\hline .5 & 317.666 & 312.5 & 8.13 \\
\hline
\end{tabular}


$T\left(r^{\prime} z^{\prime}\right)=K \iint_{I} \frac{\partial T_{g}}{\partial n}\left(r, z, r^{\prime}, z^{\prime}\right) \phi(r, z) r d r d z$

where $\partial / \partial n$ denotes differentiation with respect to the $r, z$ variables along the inward norwal to the surface over which the integration is taken. Note that the integration over $\phi$ is already incorporated into the Green's function.

Performing the indicated integration using the following integral:

$$
\int_{0}^{a} r J_{0}\left(\alpha_{n} r\right) J_{0}\left(\alpha_{m} r\right) d r= \begin{cases}\frac{a^{2}}{2}\left[J_{1}\left(a_{n} a\right)\right]^{2}, & n=m, \\ 0, & n \neq m,\end{cases}
$$

and the Fourier expansion

$$
\frac{2}{\pi} \sum_{m=1}^{\infty} \frac{m(-1)^{m} \sin \frac{m \pi z}{\ell}}{m^{2}+\frac{\ell^{2} \alpha_{1}{ }^{2}}{\pi^{2}}}=-\frac{\sinh \alpha_{1 z}}{\sinh \alpha_{1} l}
$$

gives

$$
T_{2}(r, z)=\frac{K_{1}}{K_{1}+K_{2}} \quad J_{0}(\alpha, r) \frac{\sinh \alpha_{1}(l+z)}{\sinh \alpha_{1} l \cosh \alpha_{1} l},
$$

for $0 \leq$ is $\leq a,-l \leq z<a$,

and

$T_{1}(r, z)=T_{2}(r,-z)+J_{0}(\alpha, r) \frac{\sinh \alpha_{1} z}{\sinh \alpha_{1} \ell}$,

for $0 \leq r \leq a, 0 \leq z \leq 2$. 
where $\alpha_{1}$ is the first zero of $J_{0}\left(\alpha_{n} a\right)$. This solution is verified in Appendix A.

A temperature of $80^{\circ} \mathrm{C}$ used on all external boundaries except for the face at $z=1$ which was maintained at $T(z=1)=2200 \mathrm{~J}_{0}(\alpha, r)+80$. The Monte Carlo solutions were performed using the adjoint formulation and a scaling factor of 10 . Adjoint calculations were made for the temperatures at four points along the $r=0$ axis. The results of the Monte Carlo solutions compared to the analytical results in Table 11. Each Monte Carlo calculation was made using $4.5 \mathrm{~min}$ of CPU time on the IBM $360 / 91$.

XII. TWO-MEDIA RCC WITH FUNCTIONAL INTERNAL SOURCE AND GIVEN SURFACE TEMPERATURE DISTRIBUTION

Problem 9: To caiculate the temperature distribution in a two media right circular cylinder with both an internal source and a given external temperature distribution.

For convenience, the parameters to be used for this problem are those which will give the same analytic solution as problem 6 . This is accomplished by setting the outer radius of the right circular cylinder at $a / 2$ and using the solution for problem 6 at $r=a / 2$ as the given external temperature distribution with all other parameters remaining the same.

The Monte Carlo solution was performed using the adjoint formulation and a scale factor of 10 . Adjoint calculations were made at two points along the $r=0$ axis. At $z=-.7$ the analytical solution 
TABLE 11

TEMPERATURE COMPARISONS FOR A TWO-MEDIA RCC WITH

A GIVEN SURFACE TEMPERATLRE DISTRIBUTION

\begin{tabular}{lccc}
$\begin{array}{c}\text { Analytic } \\
(\mathrm{cm})\end{array}$ & $\begin{array}{c}\text { Temperature } \\
{ }^{\circ} \mathrm{C}\end{array}$ & $\begin{array}{c}\text { Monte Carlo } \\
\text { Temperature } \\
{ }^{\circ} \mathrm{C}\end{array}$ & $\begin{array}{c}\text { Estimated } \\
\text { Standard } \\
\text { Deviation } \\
\mathrm{C}^{\circ}\end{array}$ \\
\hline .7 & 80.389 & 80.66 & .21 \\
.5 & 81.070 & 81.75 & .37 \\
.1 & 105.31 & 108.2 & 2.71 \\
\hline 5 & 278.08 & 279.2 & 6.98 \\
\hline
\end{tabular}


is $81.426^{\circ} \mathrm{C}$. The Monte Carlo solution using 4.2 min of IBM $360 / 91 \mathrm{CPU}$ time was $81.91^{\circ} \mathrm{C}$ with an estimated standard deviation of $.03 \mathrm{C}^{\circ}$. At $z=.5$ the analytical solution is $285.031^{\circ} \mathrm{C}$. The Monte Carlo solution using $4.5 \mathrm{~min}$ of IBM $360 / 91 \mathrm{CPU}$ time was $302.66^{\circ} \mathrm{C}$ with an estimated standard deviation of $.82 \mathrm{C}^{\circ}$. The Monte Carlo solution with a scale factor of 40 using $4.2 \mathrm{~min}$ of IBM $360 / 91 \mathrm{CPU}$ time was $287.76^{\circ} \mathrm{C}$ with an estimated standard deviation of $4.0 \mathrm{C}^{\circ}$.

\section{SPHERE WITH TIME DEPENDENT SOURCE}

Problem 10: To calculate the time dependent temperature distribution in a one medium sphere with a uniform, time dependent source.

Assume:

1. A sphere of radius a

2. $K$ is the thermal conductivity for $0 \leq r \leq a$

3. $\delta$ is the diffusivity for $0 \leq r \leq a$

4. Surface and initial temperature of $0^{\circ} \mathrm{C}$

5. A uniform source of $S=S_{0} e^{-\lambda t}$ for $t>0$.

The analytical solution for this problem can be obtained from Carslaw and Jaeger. 22

$$
\begin{aligned}
T(r, t)= & \frac{S_{0} \delta}{K \lambda} e^{-\lambda t} \frac{a \sin r(\lambda / \delta)^{1 / 2}}{r \sin a(\lambda / \delta)^{1 / 2}}-1 \\
& +\frac{2 a^{3} S_{0} \sum^{\infty}}{r \pi^{3} K} \frac{(-1)^{n}}{n\left(n^{2}-\lambda a^{2} / \delta \pi^{2}\right)}-\sin \frac{n \pi r}{a} e^{-\delta n^{2} \pi^{2} t / a}
\end{aligned}
$$


For numerical results, the parameters used were:

1. $\mathrm{S}_{0}=23.88 \mathrm{cal} / \mathrm{sec} \mathrm{cm}^{3}$

2. $K=.48 \mathrm{cal} / \mathrm{sec} \mathrm{cm}^{\circ} \mathrm{C}$

3. $a=20 \mathrm{~cm}$

4. $\lambda=.01 / \mathrm{sec}$

5. $\mathrm{c}=.22 \mathrm{cal} / \mathrm{g}^{\circ} \mathrm{C}$

6. $\rho=2.7 \mathrm{~g} / \mathrm{cm}^{3}$

The temperature was calculated at various times for the center of the sphere both analytically and using Monte Cario. The results of these calculations are shown in Table 12. The Monte Carlo calculations were made using the adjoint formulation. No scaling was used. For a time dependent calculation a velocity of $1 / \rho c$ is used during the tracking. An extrapolation distance correction was made for the Monte Carlo calculation. The Monte Carlo results were obtained with 2.5 min of IBM 360/91 CPU time. An advantage of the adjoint formulation for problems with time dependent sources and boundary conditions is that the solution car. be generated for many different times with the same calculation. For example, the above $2.5 \mathrm{~min}$ run performed the calculation for 59 different times.

XIV. SPHERE WITH TIME DEPENDENT SOURCE AND CONVECTIVE BOUNDARY CONDITION

Problem 11: To calculate the temperature distribution in a onemedium sphere with a convective boundary condition. 
TABLE 12

TEMPERATURE COMPARISONS FOR A SPHERE WITH

A TIME DEPENDENT SOURCE

\begin{tabular}{|c|c|c|c|}
\hline $\begin{array}{c}t \\
(\mathrm{sec}) \\
\end{array}$ & $\begin{array}{l}\text { Analytic } \\
\text { Temperature } \\
{ }^{\circ} \mathrm{C} \\
\end{array}$ & $\begin{array}{l}\text { Monte Carlo } \\
\text { Temperature } \\
{ }^{\circ} \mathrm{C}\end{array}$ & $\begin{array}{c}\text { Estimated } \\
\text { Standard } \\
\text { Deviation } \\
\mathrm{C}^{\circ}\end{array}$ \\
\hline 1 & 40.09 & 41. & 1.8 \\
\hline 5 & 196.53 & 199. & 3.6 \\
\hline 10 & 383.48 & 389. & 6.2 \\
\hline 50 & 1455.92 & 1444. & 17.3 \\
\hline 70 & 1503.05 & 1610 & 29.0 \\
\hline 80 & 1619.85 & 1621 & 34.0 \\
\hline 90 & 1605.10 & 1598 & 38.4 \\
\hline 100 & 1566.83 & 1546. & 40.2 \\
\hline 110 & $151 ! .61$ & 148.3 & 4.3 .0 \\
\hline 150 & 1210.86 & 1164. & 47.7 \\
\hline 200 & 830.41 & 787. & 45.6 \\
\hline 500 & 48.355 & 47. & .5 \\
\hline
\end{tabular}




\section{Assume :}

1. A sphere of radius a

2. $K$ is the thermal conductivity for $0 \leq r \leq a$

3. $\delta$ is the diffusivity for $0 \leq r \leq a$

4. Initial temperature is $0^{\circ} \mathrm{C}$

5. A uniform source of $S_{0}$ for $t>0$

6. Convection at $\mathrm{r}=\mathrm{a}$ into a medium at $0^{\circ} \mathrm{C}$

7. $h$ is the coefficient of convection tra.ssfer

The analytical solution can be obtained from Carslaw and Jaeger. 22 The temperature for $\mathbf{r}=0$ is given by

$T(r=0, t)=\frac{s_{0} a^{2}}{6 K}+\frac{s_{0} a}{3 h}-\frac{2 h s_{0} a^{2}}{k^{2}} \sum_{n=1}^{\infty} \frac{e^{-\delta \alpha_{t}{ }^{2} t}}{\alpha_{n} a^{2} \alpha_{n}^{2}+\frac{a h}{K}\left(\frac{a h}{K}-1\right) \sin a \alpha_{n}}$

where $\alpha_{n}$ are the roots of a $\alpha \cot a \alpha=1-a h / K$. This series converges very quickly when evaluated numerically.

To perform the Monte Carlo calculation, it is necessary to develop a method for applying the convective boundary condition to transport theory. The convective boundary conditicn for heat conduction problems car. be written:

$$
K_{1} \frac{\partial T}{\partial n}=h\left(T_{\infty}-T\right)
$$

This can be rewritten as

$$
k_{1} \frac{\partial T}{\partial n}=h \Delta n\left(\frac{T_{\infty}-T}{\Delta n}\right)
$$


If it is assumed that $T_{\infty}$ is the temperature at a distance $\Delta n$ away from the surface, then $h \Delta \mathrm{n}$ has the form of a conduction coefficient and $\left(T_{\infty}-T\right) / \Delta n$ represents the change in temperature per unit distance. Let $K_{2}=h \Delta n$. Then this boundary condition could be approximated in transport theory by adding a layer $\Delta \mathrm{n}$ thick onto the boundary with cross section $\Sigma$ given by

$$
\Sigma=\frac{1}{3 K_{2}}=\frac{1}{3 \mathrm{~h} \Delta \mathrm{n}}
$$

The thickness of this layer in mean-free-paths is given by

$$
\Sigma \Delta \mathrm{n}=\frac{\Delta \mathrm{n}}{3 \mathrm{~K}_{2}}=\frac{1}{3 \mathrm{~h}} .
$$

The thickness in mean-free-paths does not depend upon the $\Delta \mathrm{n}$ used in the approximation. If the limit as $\Delta n$ approaches 0 is taken, then it is apparent that the convective boundary condition is equivalent to a physically infinitely thin layer of material which is $1 / 3 \mathrm{~h}$ mean-freepaths thick. This type of boundary condition can be treated as an albedo

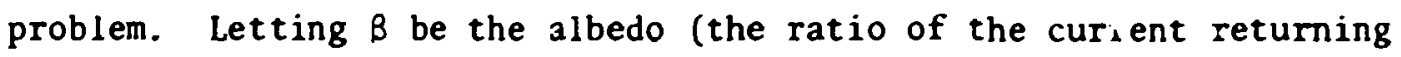
from the boundary layer to the current entering the boundary layer) and $\delta=1 / 3 \mathrm{~h}$ be the thickness of the boundary layer in mean-free-paths, a simple diffusion theory solution (based on Fick's law) for the albedo gives

$$
\beta=\frac{1-\frac{2}{3 \delta}}{1+\frac{2}{3} \delta}
$$


The paraneters for the problem are
1. $\mathrm{K}=.5 \mathrm{cal} / \mathrm{sec} \mathrm{cos}^{\circ} \mathrm{C}$
2. $h=.050 \mathrm{cal} / \mathrm{sec} \mathrm{cm}^{2 \cdot} \mathrm{C}$
3. $\rho c=.5^{\circ} \mathrm{C} / \mathrm{cal} \mathrm{c} \mathrm{cm}^{3}$
4. $S_{0}=1 \mathrm{cal} / \mathrm{cm}^{3} \mathrm{sec}$
5. $\mathbf{r}=10$

The Monte Carlo solution was performed using the adjoint formation. A scaling factor was not used. The temperature was calculated at the center of the sphere as a function of time. Using the adjoint formulation, the temperatures for all time desired can be made with the same computer run. The resuits are shown in Table 13. The Monte Carlo calculation required 4.2 min of IBM 360/91 CPU time.

The above problens have shown that the method is applicable to multimedia problems in a variety of geometries. The adjoint formulation appears to be the nost suitable for most problems.

The major source of error in most of the problems was not due to statistics but to the use of the transport equation approximation. In the next chapter the differences between the approximate sclution and the exact solution are examined to determine if corrections can be made. 
TABLF. 13

TEMPERATURE COMPARISONS FOR A SPHERE WITH A TIME DEPENDENI SOURCE AND CONVECTIVE BOUINDARY CONDITION

\begin{tabular}{|c|c|c|c|}
\hline $\begin{array}{c}t \\
(\mathrm{sec}) \\
\end{array}$ & $\begin{array}{c}\text { Analytic } \\
\text { Temperature } \\
{ }^{\circ} \mathrm{C} \\
\end{array}$ & $\begin{array}{l}\text { Monte Carlo } \\
\text { Timperature } \\
{ }^{\circ} \mathrm{C}\end{array}$ & $\begin{array}{c}\text { Estimated } \\
\text { Standard } \\
\text { Deviation } \\
\text { C }^{\circ}\end{array}$ \\
\hline$]$ & 2.000 & 2.05 & .074 \\
\hline 2 & 4.000 & 4.08 & .11 \\
\hline 3 & 6.000 & 6.15 & .15 \\
\hline 4 & 8.000 & 8.18 & .18 \\
\hline 5 & 9.996 & 10.08 & .17 \\
\hline 10 & 19.775 & 19.57 & .23 \\
\hline 20 & 37.039 & 36.17 & .33 \\
\hline 30 & 50.775 & 49.49 & .59 \\
\hline 40 & 61.535 & 60.08 & .78 \\
\hline 50 & 69.945 & 68.79 & .89 \\
\hline 100 & 91.248 & 91.46 & 1.55 \\
\hline 150 & 97.451 & 98.63 & 1.87 \\
\hline 200 & 99.258 & 100.99 & 2.02 \\
\hline
\end{tabular}

Note: The analytic steady solution as $t \rightarrow \infty$ is $100^{\circ} \mathrm{C}$. 


\section{CHAPTER V}

CORRECTIONS TO THE TRANSPORT

EQUATION APPROXIMATION

In the probleas solved in Chater IV the major source of inaccuracy was the approxination associated with using radiation particle transport theory to solve heat conduction problems. In some of the problems the inaccuracy was reduced by the use of an extrapolation distance correction as suggested by neutron diffusion theory. Problem 11, however, which involved a convective boundary condition, had no obvious inaccuracies associated with its solution. These results suggested that the transport equation approximation should be examined to determine if additional refinements could be made.

The differences between transport theory and diffusion theory can be identified as two distinct types: 1) the differences in the description of the heat flow process, and 2 j the differences in the boundary conditions which can be used.

The transport description of the heat flow process will be different from that provided by the heat conduction equation when the angular fiux calculated by the transport equation is highly anisotropic. This conditiun will occur at points which are within several mean free paths of boundaries or nonuniformly distributed sources and can be regarded as a localized effect. If an estimate of the temperature is desired at points within these regions, it wi11 often 
be possible to sale up the cross sections used in the transport calculation so that the point of interest is no longer close to a boundary or strong source when the distance is measured in mean free paths. An alternate approach is also possible if the anisotropic angular flux has azimuthal symetry about a given direction. In this case a localized one-dimensional calculation can be employed on the basis of the following analogy. The one-dimensional transport equation for the angular flux in a pure scattering medium with isotropic scattering can be written as

$$
\mu \frac{\partial \psi(x, \mu)}{\partial x}+\sum \psi(x, \mu)=\frac{\sum}{2} \int_{-1}^{1} \psi\left(x, \mu^{\prime}\right) d \mu^{\prime}+\frac{1}{2} S(x)
$$

where $\psi(x, \mu)$ is the angular flux

$\mu \quad$ is the cosine of angle between the angular flux and the

$$
x \text {-axis }
$$

$\Sigma \quad$ is the scattering cross section, and

$S(x)$ is an isotropic source.

Defining

$$
\Psi_{\ell}(x)=\int_{-1}^{1} \mu \ell \psi(x, \mu) \mathrm{d} \mu
$$

and integrating Equation (5-1) with respect to $\mu$ gives

$$
\frac{d \psi_{1}(x)}{d x}=S(x)
$$


Multiplying Equation (5-1) by $\mu$ and integrating with respect to $\mu$ gives

$$
\frac{d \psi_{2}(x)}{d x}+\sum \psi_{1}(x)=0
$$

Taking the derivative of Equation (5-4) with respect to $x$ and combining with Equation (5-3) gives

$$
\frac{1}{\Sigma} \frac{d^{2} \psi_{2}(x)}{d x^{2}}+s(x)=0
$$

Note that this is the one dimensional diffusion equation. Therefore, the second mont of the solution of the transport equation is a solution of the diffusion equation for one dimensional problems with isotropi = scattering and isotropic sources regardless of the anisotropic shape of the angular flux. This approach should therefore be useful in making calculations of temperatures in the vicinity of bnundaries or nonuniformly distributed sources.

The second kind of inaccuracy is caused by the differerces between the boundary conditions which are associated with the transpor: equation and those which can be used with the diffusion equation. These effects are not localized and represent the major source of inaccuracy in most of the problems solved in Chapter IV.

As was show in problem 11, the convective boundary condition can be represented by an equivalent albedo condition in transport theory; and results using this boundary condition are accurate. However, the fixed temperature boundary condition of heat conduction (which is 
equivalent to the nejtron diffusion theory zero flux condition) does not have an equivalent boundary condition in transport theory. The nost common nethod used is to treat the zero flux diffusion theory boundary condition as being represented by a zero incoming flux condition in the transport equation. To account for the differences in the boundary conditions, the diffusion equation flux is assumed to be zero at an extrapolated distance beyond the boundary at which the zero incoming angular flux condition is imposed. The extrapolation distance used (.71 mean free paths) is determined frow neutron transport theory. This correction was made in several of the froblems in Chapter IV and resulted in excellent agreement with the analytical solutions. However, this type of correction is not always practical for problems with complex shapes or with several different types of materials on the external boundaries. The effect of the different boundary conditions can still be minimized in cases where an extrapolation distance correction is not practical by scaling up the cross sections used in the transport calculation (with a resultant penalty of increased computer running time).

A better boundary condition for the transport solution to simulate the diffusion theory zero flux condition is obtained by considering the limit of the convective boundary condition as the thickness of the convective layer (in mean free paths) goes to zero. This limit is equivalent to the fixed temperature boundary condition. Taking this limit in Equation (4-128) (pg. 87) gives a transport condition of an albedo of minus one on the boundary. Fractional negative albedo 
conditions can be used in transport solutions but a negative albedo of minus one on all external boundaries is nonconvergent. To test the negative albedo boundary condition, various negative albedo conditions were applied at the boundary of the Milne problea and solution obtained with ANISN. 28 The Milne problen is ans dinensional infinite half-space problen (material from 0 to $\infty$ ) with a unit source at infinity. The albedo condition was ilposed at the boundary $x=0$. The results of the ANISN calculations for the total flux are given in Figure 8 for albedo conditions of 0 and -.4 , and are colpared to the diffusion theory solution with a zero flux boundary condition. Based on these calculations, if the change assoclated with going from a vacuum boundary condition (zero inconing angular flux) to a -.4 albedo condition is muliplied by 1.89 and added to the vacuum boundary transport solution, the results are the same as the diffusion theory solution at points which are not close to the boundary. The results are also very good even at points close to the boundary. An albedo of -.4 was chosen because it gave a significant correction (over $50 \%$ of the difference between the vacuum boundary transport theory solution as compared to the zero flux diffusion theory solution) and is still small enough so as to not prohibitively increase the running time for problem solutions. Test problems with a -.4 albedo condition ran 1.5 to 2.0 times as long as those with a vacuum boundary condition. This type of b.oundary condition is easily implemented in Monte Carlo transport codes and can be implemented so as to give the solution with a vacucm boundary condition and the negative albedo boundary condition in the same run. 


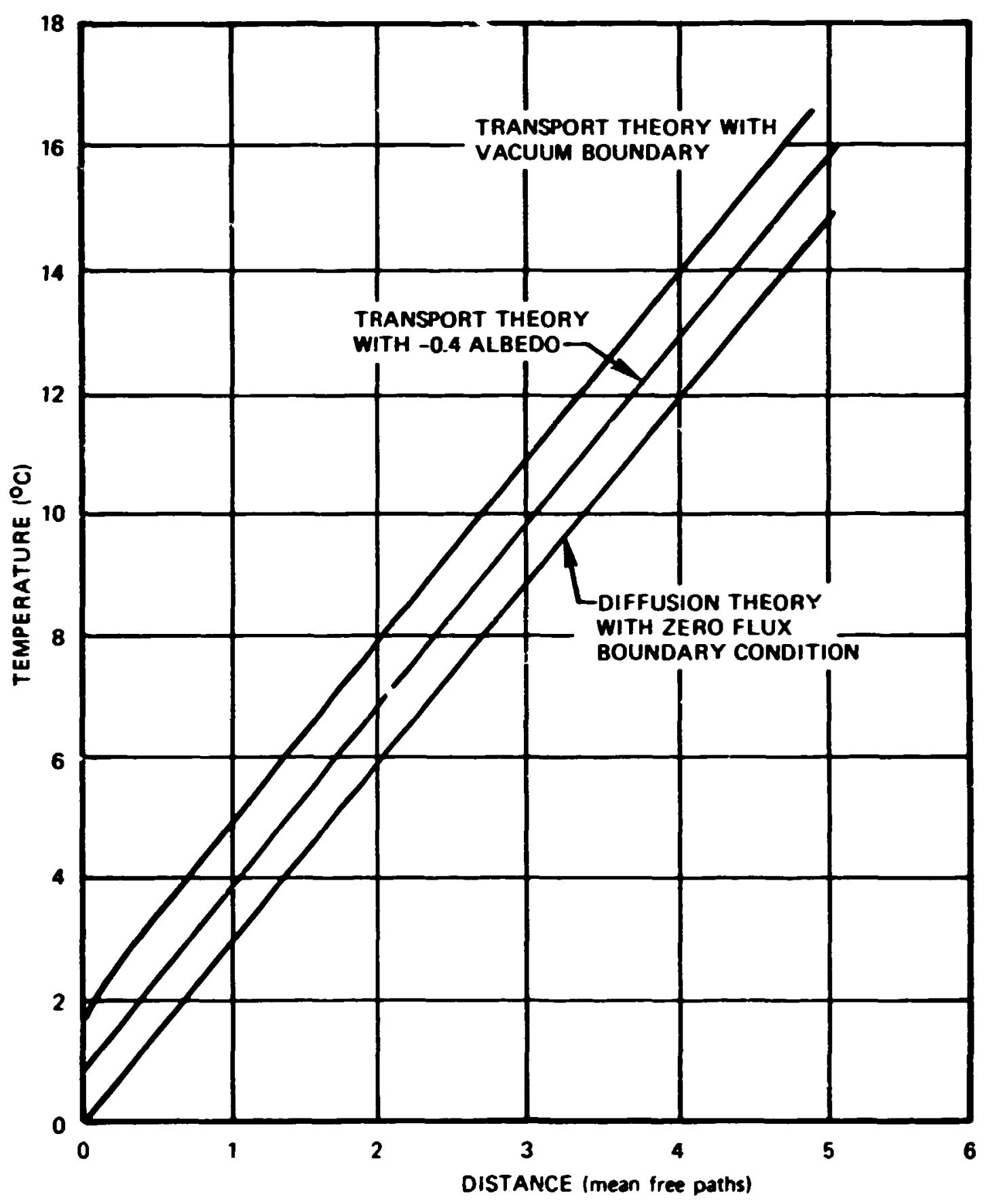

Figure 8. Diffusion theory vs transport theory with different boundary conditions. 
The negative albedo boundary condition was used to recalculate problen 2. The results of these calculations are shown in Table 14. The Monte Carlo solution was performed using the adjoint formation and a scaling factor of 10 . With the exception of the negative albedo boundary condition, the transport theory results vere performed in the same manner as the calculations in Table 4 (p. 42). Since the estinated standard deviation for the results at $x=-.7$ ca were larger than desired to make an adequate evaluation of the effect of the negative albedo boundary condition, a second calculation was perforned using 19 min of IBM $360 / 91$ CPU time. The results of this calculation was a Monte Carlo estimate of $81.86^{\circ} \mathrm{C}$ with an estinated standard deviation of $.18 \mathrm{C}^{\circ}$ as compared to the analytical results of $81.83^{\circ} \mathrm{C}$. Using the above correction factor, the major source of error is now the statistical error associated with the Monte Carlo process. 
TABLE 14

TEMPERATURE COAPAKISOIS FOR PROBLENI TWO WITH

TIIF. NEGATILE ALBEDO CORRECTION FACTOR

\begin{tabular}{cccc}
\hline $\begin{array}{c}\text { Analytic } \\
(\mathrm{cm})\end{array}$ & $\begin{array}{c}\text { Temperature } \\
{ }^{\circ} \mathrm{C}\end{array}$ & $\begin{array}{c}\text { Aonte Carlo } \\
\text { Temperaiure } \\
{ }^{\circ} \mathrm{C}\end{array}$ & $\begin{array}{c}\text { Estimated } \\
\text { Standard } \\
\text { Deviat:on } \\
\mathrm{C}^{\circ}\end{array}$ \\
\hline-.7 & 81.83 & 81.79 & .31 \\
-.5 & 84.73 & 31.79 & .62 \\
.1 & 166.2 & 169.5 & 2.6 \\
\hline .5 & 272.5 & 271.6 & 3.3 \\
\hline
\end{tabular}


CHAPTER VI

MONTE CARLO SOLUTION OF A COUPLED GAMA RADIATION

AND HEAT CONDUCTION PROBLEM

One of the major advantages of this method of solution of the heat conduction equation is that it will allow the solution of coupled nuclear radiation transport and heat i onduction problems where nuclear radiation interactions with matter are the heat source for the heat conduction problem. Another important advantage is the capability of solving these problems in complex geometries. In this section the Monte Carlo method is applied to the solution of a coupled gama radiation and heat conduction problem.

Problem 12: To calculate the temperature at several points in a sphere when the heat source is an incident parallel beam of ganma radiation (Figure 9).

Assumptions and Parameters:

1. Outer boundary of the sphere is maintained at a constant temperature.

2. Radius of sphere is $10 \mathrm{~cm}$.

3. $\mathrm{K}=.3333 \mathrm{cal} / \mathrm{cm} \mathrm{sec}{ }^{\circ} \mathrm{C}$.

4. $\Sigma_{t}$ for gammas (all energies) $=.1 \mathrm{~cm}^{-1}$.

5. $\Sigma_{a}$ for gammas $=.03 \mathrm{~cm}^{-1}$.

6. $1 \mathrm{cal} / \mathrm{cm}^{2} \mathrm{sec}$ energy in the incident gamma radiation.

7. Steady-state condition.

8. Isotropic scattering of gamma radiation. 

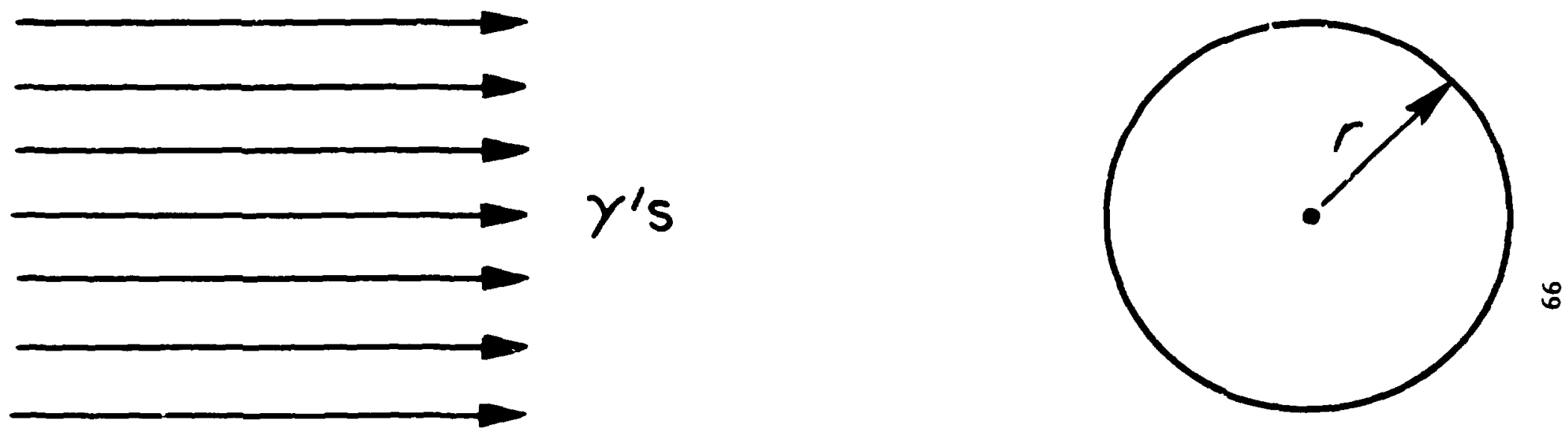

Figure 9. One-medium sphere with incident ganma beam. 
The values chosen are typical of those for a metal such as aiuminum.

To provide a check on tine Monte Carlo solution, the average temperature as anction of radius of the sphere is estimated using previously available techniques. For calculating the average gama flux as a function of radius, $\mathrm{Kalos}^{29}$ has shown that the incident parallel beam of $\phi_{0}$ particles/ $\mathrm{cm}^{2}-\mathrm{sec}$ can be replaced by an isotropic flux of $\phi_{0}$ particles/ $\mathrm{cm}^{2}-\mathrm{sec}$ over the entire surface of the sphere. Using this transformation, the average gamma fiux as a function of radius was solved using the discrete ordinates code ANISN ${ }^{28}$ and the average energy deposition was calculated. With an incident gamma radiation beam of $1 \mathrm{cal} / \mathrm{cm}^{2}-\mathrm{sec}$, the radius averaged gamma flux was fairly uniform in the sphere varying from a low of $.685 \mathrm{cal} / \mathrm{cm}^{2}-\mathrm{sec}$ at the center to a high of $.848 \mathrm{cal} / \mathrm{cm}^{2}-\mathrm{sec}$ at the edge. The volume averaged flux was $.773 \mathrm{cal} / \mathrm{cm}^{2}-\mathrm{sec}$. The average heat source in the sphere is then given by $\phi_{\text {avg }} \sum_{a}$ and is $.0232 \mathrm{cal} / \mathrm{cm}^{3}-\mathrm{sec}$. Assuming that this heat source is uniform across the sphere, an estimation of the temperature as a function of radius can then be made analytically.

The Monte Carlo cajculation of the coupled gamma radiation and heat conduction problem was performed using MORSE-SGC. The logic used for performing a coupled gamma radiation and heat conduction problem with MORSE-SGC is the same as that used for solving a coupled neutrongamma problem. For the forward formilation of the problem, a gamma starting location is chosen from the parallel bean distribution and the ganma particle is tracked until it escapes or until the weight of the 
particle has been reduced by nonabsorption weighting at collision sites and its survival determined by Russian roulette. At each collision site energy is absorbed and represents a heat source. This heat source is treated as a secondary particle. In the actual calculation a secondary particle was generated with a probability of .1 at each collision site with particle weighting to maintain a fair game. The secondary particles (the heat sourre) are then tracked to determine temperatures. As was expected, an adjoint formulation of the problem was necessary to obtain good statistics. The adjoint calculation obtained fractional standard deviations of less than $1 \%$ in the same time that the forward calculation obtained fractional standard deviations of over $30 \xi$ ). A next event estimator was used in the adjoint formulation at each gamna collision site (the secondary particles in the adjoint formulation) and the probability of the gamma having reached this collision site from the incident beam was scored. By scoring to different detectors, where for each detector a different location of the incoming beam was used, the temperature at a number of points could be calculate 1 with the same computer run. Each of these points will have the same radius but are at locations which have the same relative locations to the incoming beams as was used during the estimator process.

Comparisons of the analytical estimation of the average temperature as a function of radius and the Monte Carlo calculation of the temperature at various locations in the sphere are shown in Tables 15-19 and Figures 10-14. An adjoint Monte Carlo calculation was performed for each of the seven radii shown in the figures and 
TABLE 15

TEMPERTURES FOR $\theta=0^{\circ}$ COMPARED TO AIALYTICAL ESTIMATE

\begin{tabular}{cccc}
\hline$r$ & $\begin{array}{c}\text { Analytical } \\
\text { Estimate } \\
(\mathrm{cm})\end{array}$ & $\begin{array}{c}\text { Monte Carlo } \\
\text { Calculation }\end{array}$ & $\begin{array}{c}\text { Estimated } \\
\text { Standard } \\
\text { Deviation } \\
{ }^{\circ} \mathrm{C}\end{array}$ \\
\hline 0 & 1.16 & 1.05 & .021 \\
1.0 & 1.15 & 1.13 & .026 \\
2.0 & 1.11 & 1.13 & .024 \\
3.0 & 1.056 & 1.15 & .023 \\
4.0 & .974 & 1.15 & .033 \\
5.0 & .371 & 1.03 & .023 \\
7.5 & .508 & .640 & .019 \\
9.0 & .220 & .312 & .019 \\
\hline
\end{tabular}

Note: The fonte Carlo temperatures are calculated along a radius which makes an angle $\theta$ with the direction towards the incident gamma radiation.

TABLE 16

TEMPERATURES FOR $\theta=45^{\circ}$ COMPARED TO ANALYTICAL ESTIMATE

\begin{tabular}{lccc}
\hline $\begin{array}{c}\text { Analytical } \\
\text { Estimate } \\
{ }^{\circ} \mathrm{C}\end{array}$ & $\begin{array}{c}\text { Monte Carlo } \\
\text { Calculation }\end{array}$ & $\begin{array}{c}\text { Fstimated } \\
\text { Standard } \\
\text { Deviation } \\
\mathrm{C}^{\circ}\end{array}$ \\
\hline 0 & 1.16 & 1.05 & .021 \\
1.0 & 1.15 & 1.12 & .024 \\
2.0 & 1.11 & 1.14 & .023 \\
3.0 & 1.056 & 1.11 & .023 \\
4.0 & .974 & 1.12 & .032 \\
5.0 & .871 & .988 & .021 \\
7.5 & .508 & .611 & .018 \\
9.0 & .220 & .297 & .018 \\
\hline
\end{tabular}

Note: The Monte Carlo temperatures are calculated along a radius which makes an angle $\theta$ with the direction towards the incident gamma radiation. 
TABLE 1;

TER:PERATURES FOR $\theta=90^{\circ}$ COHPARED TO A:FALYTICAL ESTIPLATE

\begin{tabular}{lccc}
\hline $\begin{array}{c}\text { Analytical } \\
(\mathrm{cm})\end{array}$ & $\begin{array}{c}\text { Estinate } \\
{ }^{\circ} \mathrm{C}\end{array}$ & $\begin{array}{c}\text { Ionte Carlo } \\
\text { Calculation } \\
{ }^{\circ} \mathrm{C}\end{array}$ & $\begin{array}{c}\text { Estimated } \\
\text { Standard } \\
\text { Deviation } \\
\mathbf{C}^{6}\end{array}$ \\
\hline 0 & 1.16 & 1.05 & .021 \\
1.0 & 1.15 & 1.09 & .025 \\
2.0 & 1.11 & 1.07 & .021 \\
3.0 & 1.056 & 1.00 & .024 \\
4.0 & .974 & .987 & .031 \\
5.0 & .371 & .353 & .020 \\
7.5 & .508 & .497 & .015 \\
9.0 & .220 & .238 & .016 \\
\hline \hline
\end{tabular}

Vote: The ilonte Carlo temperatures are calculated along a radius which makes an angle $\theta$ with the direction towards the incident gama radiation.

TABLE 13

TEMPERATURES FOR $\theta=135^{\circ}$ COMPARED TO A:IALYTICAL ESTIMATE

\begin{tabular}{|c|c|c|c|}
\hline $\begin{array}{l}\mathrm{r} \\
(\mathrm{cm})\end{array}$ & $\begin{array}{c}\text { Analytical } \\
\text { Estimate } \\
{ }^{\circ} \mathrm{C} \\
\end{array}$ & $\begin{array}{l}\text { Monte Carlo } \\
\text { Calculation } \\
{ }^{\circ} \mathrm{C}\end{array}$ & $\begin{array}{c}\text { Estimated } \\
\text { Standard } \\
\text { Deviat ion } \\
C^{\circ}\end{array}$ \\
\hline 0 & 1.16 & 1.05 & .021 \\
\hline 1.0 & 1.15 & 1.05 & .025 \\
\hline 2.0 & 1.11 & .993 & .021 \\
\hline 3.0 & 1.056 & .904 & .024 \\
\hline 4.0 & .974 & .335 & .027 \\
\hline . 0 & .871 & .69\%. & .020 \\
\hline$" .5$ & .508 & .348 & .013 \\
\hline 9.0 & .220 & .154 & .012 \\
\hline
\end{tabular}

Note: The Monte Carlo temperatures are calculated along a radius which makes an angle $\theta$ with the direction towards the incident gamma radiation. 
TABLE 19

TEMPERATURES FOR $e=180^{\circ}$ COMPARED TO A:IALYTICAL ESTIHATE

\begin{tabular}{lccc}
$\begin{array}{c}\text { Analytical } \\
\text { Estimate } \\
\left.{ }^{\circ} \mathrm{Cm}\right)\end{array}$ & $\begin{array}{c}\text { Monte Carlo } \\
\text { Calculation }\end{array}$ & $\begin{array}{c}\text { Estimated } \\
\text { Standard } \\
\text { Deviation } \\
\mathrm{C}^{\circ}\end{array}$ \\
\hline 0 & 1.16 & 1.05 & .021 \\
1.0 & 1.15 & 1.04 & .026 \\
2.0 & 1.11 & .963 & .022 \\
3.0 & 1.056 & .867 & .024 \\
4.0 & .974 & .760 & .025 \\
5.0 & .871 & .630 & .023 \\
7.5 & .508 & .239 & .012 \\
9.0 & .220 & .127 & .011 \\
\hline
\end{tabular}

Note: The Monte Carlo temperatures are calculated along a radius which makes an angle $e$ with the direct: on towards the incident gamna radiation. 


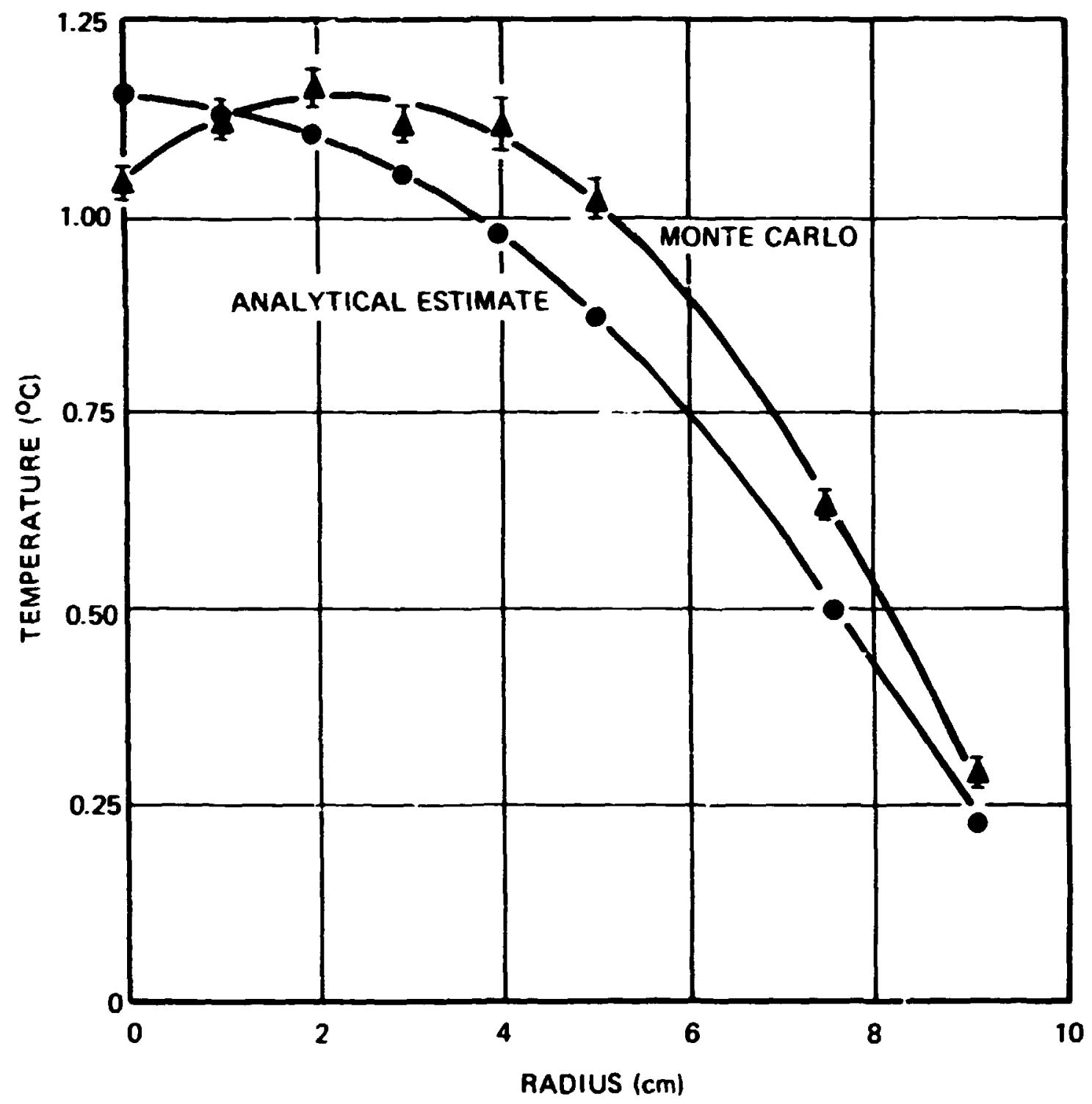

Figure 10. Temperatures for $\theta=0^{\circ}$ compared to analytical estimates. 


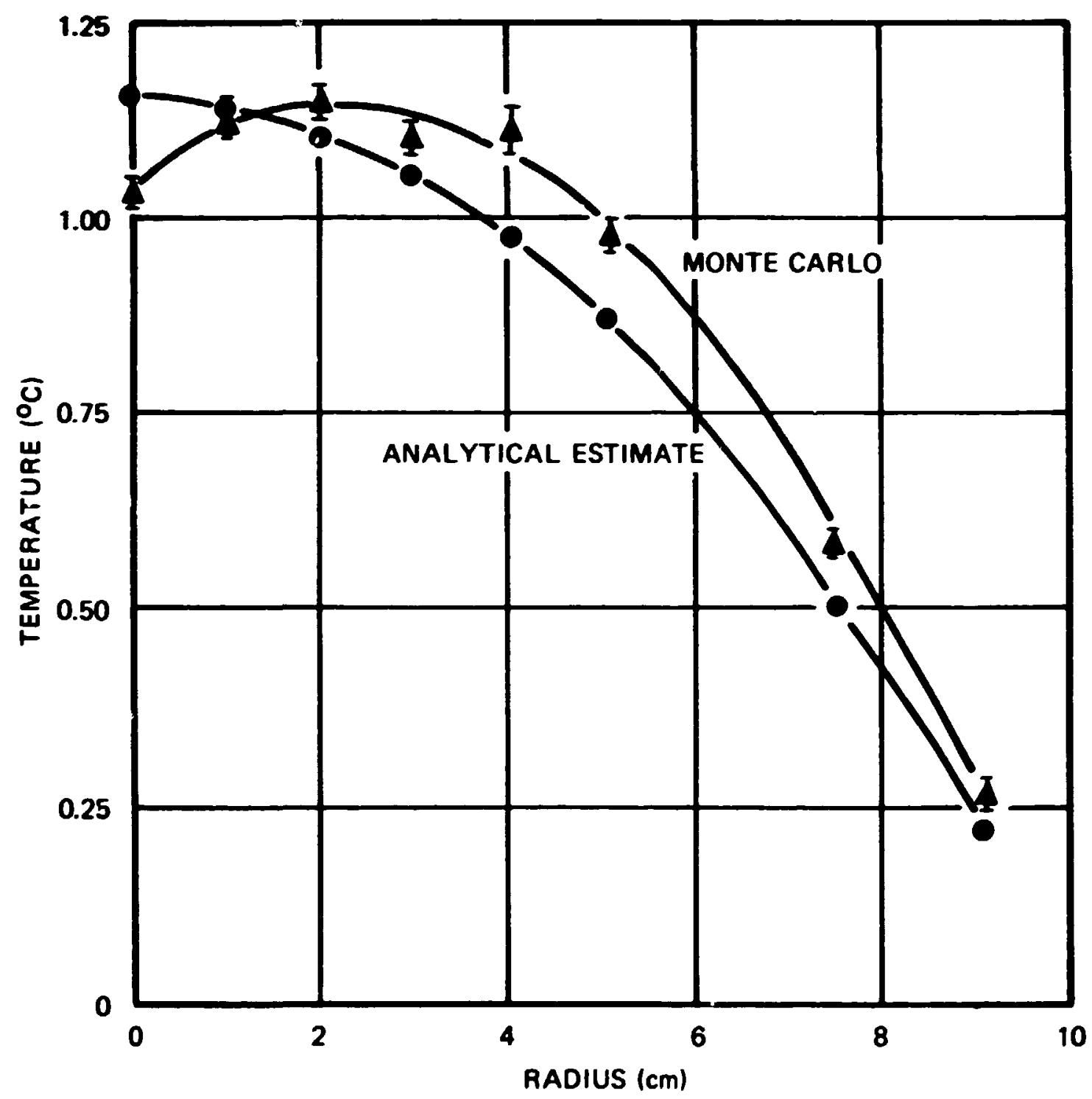

Figure 11. Temperatures for $\theta=45^{\circ}$ compared to analytical estimates. 


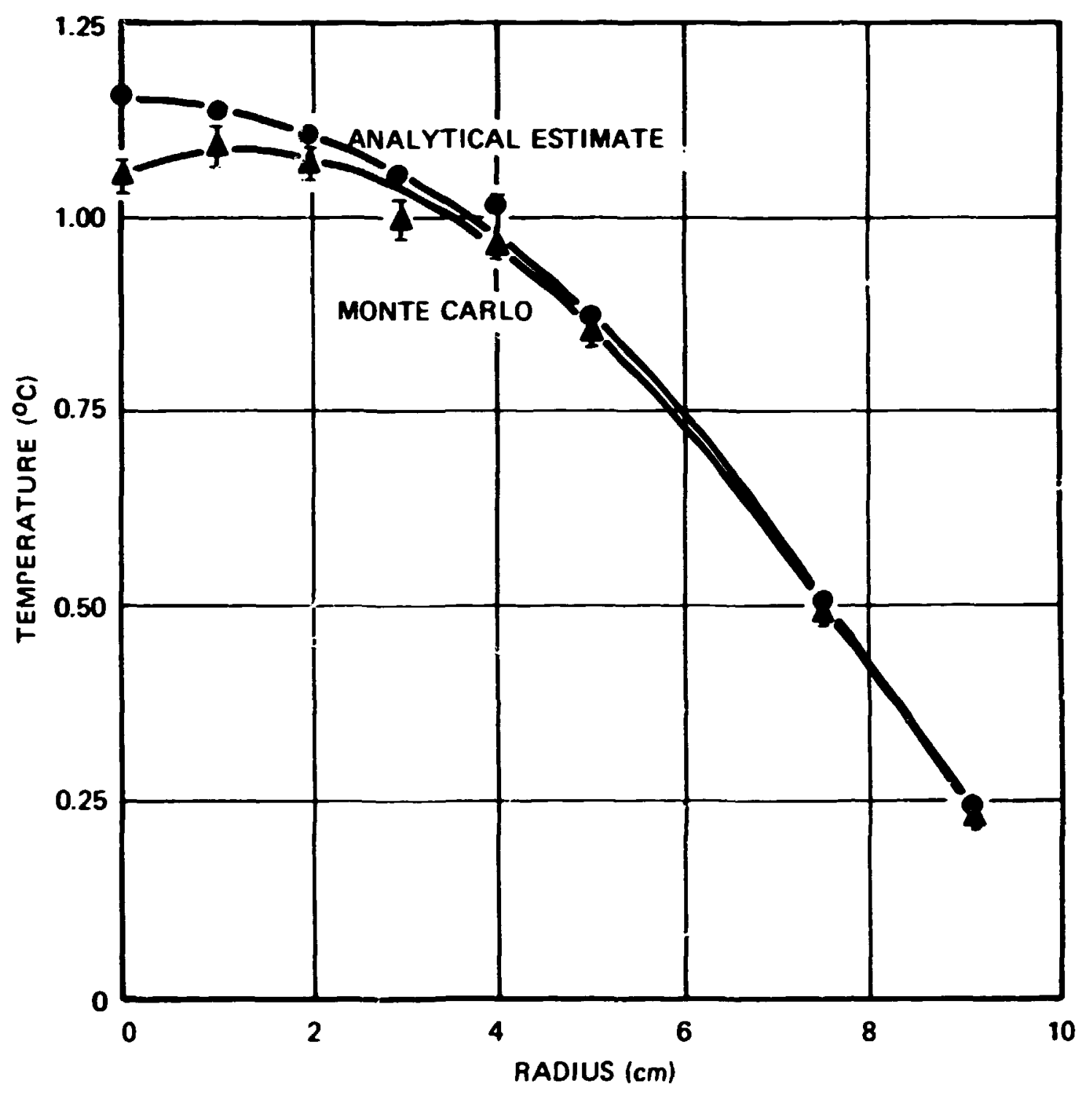

Figure 12. Temperatures for $\rho=90^{\circ}$ compared to analytical estimates. 


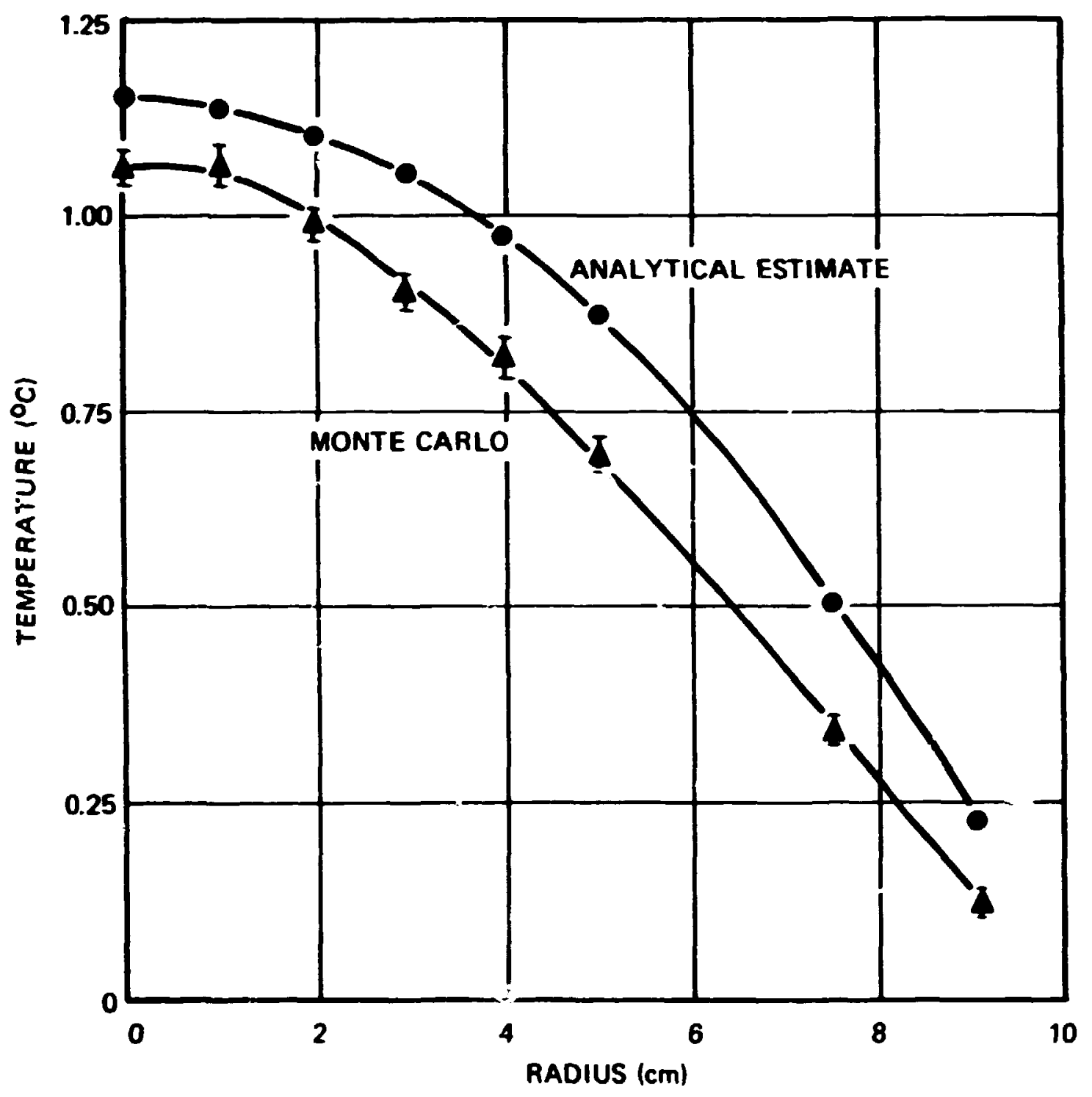

Figure 13. Temperatures for $\theta=135^{\circ}$ compared to analyticai estimates. 


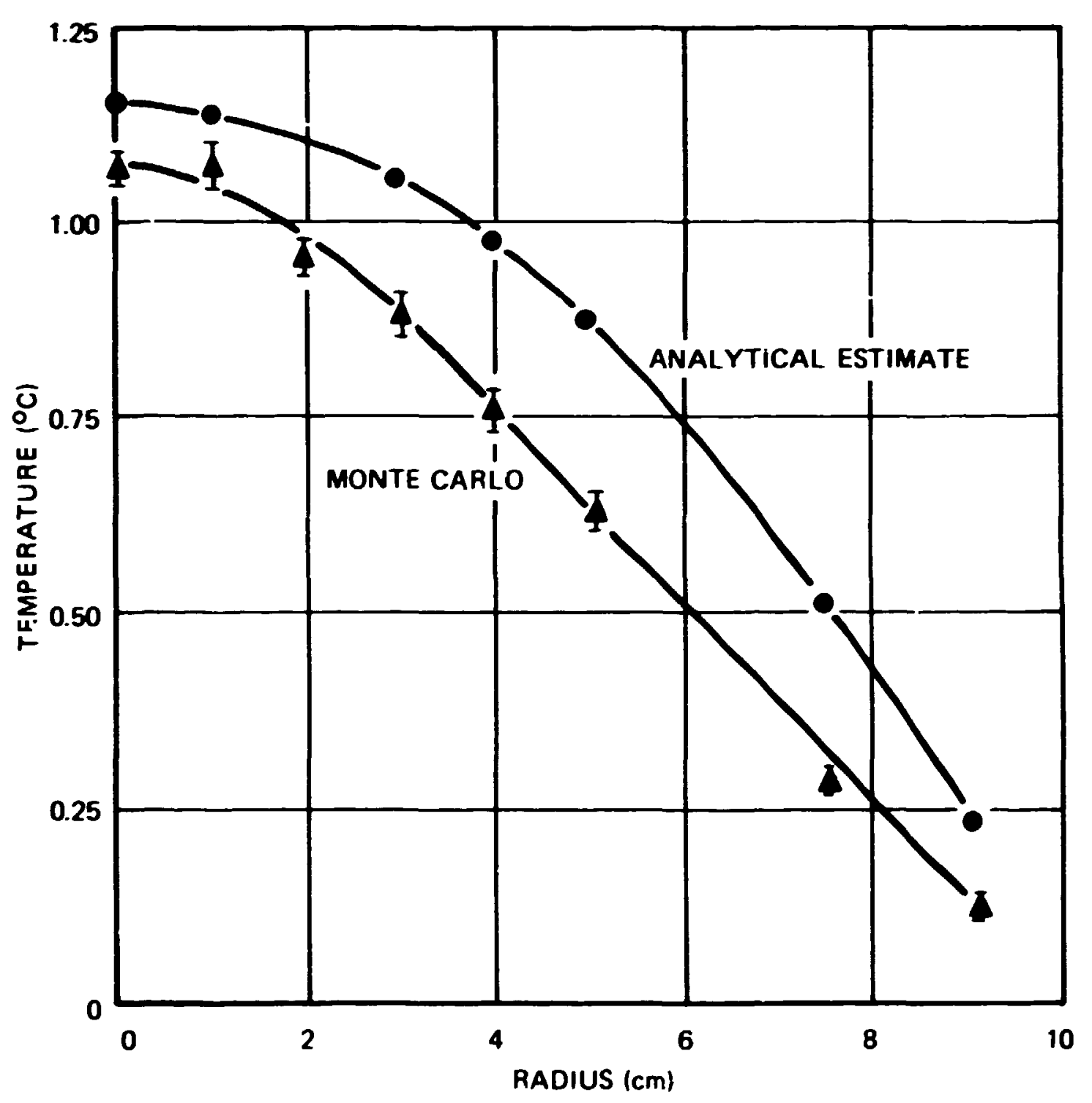

Figure 14. Temperatures for $\theta=180^{\circ}$ compared to analytical estimates. 
tables. Each monte Carlo calculation required 4.2 min of CPU time on the IBM 360/91. A listing of the input to MORSE-SCC and LAJA, 30 and the relevant output is given in Appendix $C$ for the calculation at $\mathbf{r}=2.0 \mathrm{~cm}$. LAVA was used to create the necessary cross sections for MORSE-SGC.

The analytical estimate of the average te:perature at a given radius differs from the Monte Carlo calculation of the same quantity because the analytical results are based on the assuaption that the heat generation within the sphere is uniform. This is a fairly good approximation but is not exact. Since the heat generation is higher near the adges than at the center, the correct temperature at the center is lower than the analytical estimate.

The Monte Carlo results along various radii show the variation of the temperature from the average over the sphere. As is expected intuitively, the hottest point in the sphere is not at the center but is several centimeters off center in the direction towards the incident bean of gamma radiation. 


\section{CONCLUSIONS AND RECOMENDATIONS}

The transport theory approxination has been shown to be appropriate for use in solving many types of heat conduction problems. As was demonstrated in the problems, the transport approximation is not limited to Monte Carlo methods. However, since most other techniques can be used to solve the heat conduction equation directly, the approximation is not necessary with these other methods.

As is typical with current Monte Carlo methoos, other methods of problem solutions appear to be preferable when they may be used. However, there are many heat conduction problems for which the solution is not feasible using other methods. The application of the Monte Carlo method to the transport equation approximation will provide solutions to many of these rroblems. There are no inherent limitations as to the geometric complexity of the heat conduction problems which can be solved using this method. It will also simplify, or make possible, the solution of coupled problems which involve nuclear reactions as the heat source for a heat conduction problem.

The Monte Carlo solution of the transport equation approximation is more suitable for calculating temperatures at individua! points within a system rather than calculating complete temperature distributions. The adjoint formulation appears to be the most suitable mode of solution for most problems. The method is not applicable to problems where the thermal conductivity is temperature dependent. 
The advantages and disadvantages of the Monte Carlo solution of the transport equation approximation are illustrated by the following cosparison to the surface density walk and floating random walk techniques:

1. There are no published results on the use of either the surface density walk or floating randon walk in multimedia problems.

2. The tracking algorithm used by the floating random walk is difficult since it requires the determination of the nearest boundary point. Both the transport equation apprcximation and the surface density walk use a simpler method which requires the finding of the intersect point between a surface and a given line.

3. The surface density walk tracks directly to surface points, with the number of tracks for a given walk being determined by the number of terms in the truncated Fredholm series being evaluated. The floating random walk tracks over large distances until the walk approaches a boundary in which case the di.jtances become smaller and smaller. An approximation must then be used to reach the boundary. The transport equation approximation uses relatively small steps throughout the walk with the step size becoming smaller as a scale factor is increased to make the transport equation approximation better.

4. The surface density walk and the transport equation approximation may be used with or readily adapted to existing Monte Carlo radiation transport codes. 
5. All three methods may be used to solve hear conduction problems with internal heat sources, given surface temperature distributions and time dependence.

6. The floating random walk and the transport equation approxilation may be used to solve problems with convective boundary conditions.

7. The transport equation approxiduation may be used to soive coupled nuclear radiation and heat conduction problems.

The Monte Carlo solution of the transport equation approximation has been shown to be useful in the solution of many types of heat conduction problems. However, the following areas still require further research.

1. Contact resistance between meria.

This type of problem should be solvable by the use of an appropriate albedo medium at the contact surface to transmit or reflect a fixed ratio of particles striking the surface. This technique still requires demonstration, however.

2. Other boundary conditions.

In Chapter II, many different types of standard boundary conditions are listed for heat conduction problems. Only the fixed surface temperature distribution and the convective boundary condition problems were solved. Further research is needed to determine effective means of handling the remaining types of boundary conditions. 
3. Biasing techniques.

All of the standard biasing techniques available for use in sclving transport problews with Monte Carlo are applicable to this method. Further research is still needed, however, to determine how these techniques may be effectively applied in the use of transport problems which approximate heat conduction problems.

4. Coupling with deterministic codes.

The method should also be useful when used in conjunction with deterministic codes to perform perturbation calculation or to couple the Monte Carlo calculation to deterministic codes as in the adjoint difference method. 31 Further research is needed to establish effective and convenient methods to accomplish the coupling with heat conduction codes. 
BIBLIOGRAPHY 


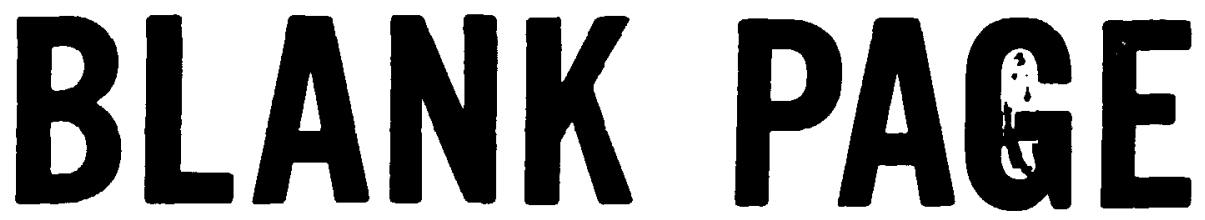




\section{BIBLIOGRAPHY}

1. T. J. Heffman and N. E. Banks, "Monte Carlo Surface Density Solution to the Dirichlet Heat Transfer Problem," Nucl. Sci. E Eng. , 59, 205-214 (1976).

2. A. Haji-Sheikh and E. M. Sparrow, "The Solution of Heat Conduction Problems by Probability Methods," J. Heat Transfer, Trans. ASME, 89, 121 (1967).

3. A. Haji-Sheikh and E. M. Sparrow, "The Floating Random Walk and Its Application to Monte Carlo Solutions of Heat Equations," J. SIAM, App 1. Math., 14, 370 (1966).

4. E. S. Troubetzkoy and N. E. Banks, "Solution of the Heat Diffusion Equa: i on by Monte Carlo," Trans. Am. Nucl. Soc., 19, 163 (1974).

5. T. J. Hof fman and N. E. Banks, "Monte Carlo Solution to the Dirichlet Problem with the Double Layer Potential Density," Trans. Am. Nucl. Soc., 18, 136 (1974).

6. N. E. Banks and T. J. Hoffman, "Continuous Monte Carlo Solution to the Dirichlet Heat Transfer Problem Using the Double Layer

Potential," 20th Army Math. Conf., Boston, Mass. (1974).

7. N. E. Banks and T. J. Hoffman, "Extension of the Surface Density Monte Carlo Technique to Heat Transfer in Non-Convex Regions," Trans. Am. Nucl. Soc., 19, 164 (1974).

8. T. J. Hoffman and N. E. Banks, "Monte Carlo Solutions to Transient Heat Conduction Problems," Trans. Am. Nuc1. Soc., 21, 204 (1975).

9. T. J. Horfman, "Monte Carlo Solution to Heat Conduction Problems with internal Sources," Trans. Am. Nucl. Soc., 24, 181 (1976).

10. M. E. Muller, "Some Continuous Monte Carlo Methods for the Dirichlet Problem," Ann. Math. Stat., 27, 569-589 (1956).

11. J. J. Thompson and P. Y. P. Chen, "Heat Conduction with Internal Sources by Modified Monte Carlo Method," Nucl. Engr. and Design, $12,207-214$ (1970).

12. J. Todd, "Experiments in the Solution of Differential Equations by Monte Carlo Methods," J. of the Wash. Acad. of Sci., 44 (1954).

13. S. Hoshino and K. Ichida, "Solution of Partial Differential Equations by a Modified Random Walk," Numer. Math., 18, 61-72 (1971). 
14. W. D. Little, "Hybrid Computer Solutions of Partial Differential Equation by Monte Carlo Method," Ph.D. thesis, University of Eritish Columbia (1965).

15. L. Fahreir, "Extension of the Hybrid Monte Carlo Method for Boundary-Value Problems," Sinulation, 21(6), 477 (1973).

16. M. N. Ozisik, "Boundary Value Problens of Heat Conduction," Scranton, International Textbook Co. (1968).

17. R. L. Murray, "Nuclear Reactor Physics," Princeton Hall Publishing Co., Inc. (1957).

18. K. M. Case, F. de Hoffian and G. P. Placzek, "Introduction to the Theory of Neutron Diffusion," U.S. Gov't Printing Office, Washington, D.C. (1953).

19. J. Spanier and E. M. Gelbard, "Monte Carlo Principles and Neutron Transport Problems," Addison-Wesley Publishing Co., Inc. (1969).

20. L. L. Carter and E. D. Cashwe11, "Particle-Transport Simulation with the Monte Carlo Method," USERDA Tech. Info. Center, TID-26607 (1975).

21. R. R. Coveyou, V. R. Cain and K. J. Yost, "Adjoint and Importance in Monte Carlo Application," Nucl. Sci. E Eng., 27, 219-234 (1967).

22. H. S. Carslaw and J. C. Jaeger, "Conduction of Heat in Solids," Oxford, 2nd ed. (1959).

23. S. K. Fraley, "User's Guide to MORSE-SGC," ORNL/CSD-7 (1976).

24. M. H. Kalos, "On the Estimation of Flux at a Point by Monte Carlo," Nucl. Sci. E Eng. 16, 111-117 (1963).

25. H. A. Steinberg and M. H. Kalos, "Bounded Estimators for Flux-ata-Point in Monte Car10," Nucl. Sci. \& Eng., 44, 406 (1971).

26. H. A. Steinberg and H. Lichtenstein, "Implementation of Bounded Point Estimators in Point Cross-Section Monte Carlo Codes," Trans. A. Nucl. Soc., 17, 259 (1973).

27. W. A. Rhoades and F. R. Mynatt, "The DOT-III Two Dimensional Discrete Ordinates Transport Code," ORNL-TM-4280 (1973).

28. W. W. Engle, Jr., "A User's Manual for ANISN, A One-Dimensional Discrete Ordinates Transport Code with Anisocropic Scattering," UCC-ND, K-1693 (1967). 
29. M. H. Kalos, "ANTE 2, Adjoint Monte Carlo Time-Dependent Neutron Transport Code in Combinatorial Geometry," Appendix G, MAGI Inc. (1970).

3J. S. K. Fraley, "A User's Guide for LAVA as Issued with MORSE-SGC," ORNL/CSD-11 (1976).

31. T. J. Hoffman, J. C. Robinson and P. H. Stevens, "The Adjoint Difference Method and Its Application to Deep Penetration Radiation Transport," Nucl. Sci. E Eng., 48, 179-188 (1972). 


\section{APPENDIXES}


APPESDIX A

VERIFICATION OF AHALYTICAL SOLUTIONS

Once an analytical solution has been developed for a heat conduction problem, the solution may be verified by showing that it satisfies the heat conduction equation and the boundary corditions. Many of the problens presentcd in Chapter IV involved the derivation of an appropriate Green's function to obtain the analytical solution. Since a large number of manipulations (which could introduce errors) were involved in the development of the solutions, a verification of the more difficult solutions is given in this appendix.

The analytical solution to problen $\bar{z}$ is

$$
\begin{aligned}
& T_{2}(x, y, z)=\frac{S_{0} a}{\pi^{2}} \cdot \frac{1}{K_{1}+K_{2}} \cdot \frac{1}{1+a^{2}\left(\frac{1}{b^{2}}+\frac{1}{c^{2}}\right)} \cdot \frac{1}{\left(\frac{1}{b^{2}}+\frac{1}{c^{2}}\right)^{1 / 2}} \cdot \sin \frac{\pi y}{b} \sin \frac{\pi z}{c} \\
& \frac{\sinh \pi\left[(a+x)\left(\frac{1}{b^{2}}+\frac{1}{c^{2}}\right)^{1 / 2}\right]}{\cosh \left[\pi a\left(\frac{1}{b^{2}}+\frac{1}{c^{2}}\right)^{1 / 2}\right]}
\end{aligned}
$$

for $-a \leq x \leq 0,0 \leq y \leq b, 0 \leq z \leq c$, and

$$
T_{1}(x, y, z)=T_{2}(-x, y, z)+\frac{S_{0}}{\pi^{2} K_{1}} \cdot \frac{1}{\frac{1}{a^{2}}+\frac{1}{b^{2}}+\frac{1}{c^{2}}} \cdot \sin \frac{\pi x}{a} \sin \frac{\pi y}{b} \sin \frac{\pi z}{c}
$$

for $0 \leq x \leq a, 0 \leq y \leq b$, and $0 \leq z \leq c$. 
Since the problen is steady state, the solution must satisfy the following:

(1) $\nabla^{2} T=-\frac{s(x, y, z)}{K}$,

where

$$
S(x, y, z)=s_{0} \sin \frac{\pi x}{a} \sin \frac{\pi y}{b} \sin \frac{\pi z}{c},
$$

for $0 \leq x \leq a, 0 \leq y \leq b, 0 \leq z \leq c$, zero elsewhere,

(2) $T(x, y, z)=0$,

for all $(x, y, z)$ on outer boundaries, and

(3) $K_{1} \frac{\partial T_{1}}{\partial x}=K_{2} \frac{\partial T_{2}}{\partial x}$,

at the interface between the two media.

By inspection it is obvious that $T(x, y, z)=0$ at outer boundaries. To evaluate $\nabla^{2} T_{2}(x, y, z)$, let

$$
T_{2}(x, y, z)=a \sin \frac{\pi y}{b} \sin \frac{\pi z}{c} \sinh \left[\pi(a+x)\left(\frac{1}{b}+\frac{1}{c^{2}}\right)^{1 / 2}\right] .
$$

Then

$$
\frac{\partial^{2} T_{2}}{\partial y^{2}}=-\alpha\left(\frac{\pi}{b}\right)^{2} \sin \frac{\pi y}{b} \sin \frac{\pi z}{c} \sinh \left[\pi(a+x)\left(\frac{1}{b}+\frac{1}{c^{2}}\right)^{1 / 2}\right],
$$




$$
\frac{\partial^{2} T_{2}}{\partial z^{2}}=-a\left(\frac{\pi}{c}\right)^{2} \sin \frac{\pi y}{b} \sin \frac{\pi z}{c} \sinh \left[\pi(a+x)\left(\frac{1}{b}+\frac{1}{c^{2}}\right)^{1 / 2}\right],
$$

and

$$
\frac{\partial^{2} T_{2}}{\partial x^{2}}=a \pi^{2}\left(\frac{1}{b^{2}}+\frac{1}{c^{2}}\right) \sin \frac{\pi y}{b} \sin \frac{\pi z}{c} \sinh \left[\pi(a+x)\left(\frac{1}{b^{2}}+\frac{1}{c^{2}}\right)^{1 / 2}\right] \cdot(A-10)
$$

Therefore,

$$
\nabla^{2} T_{2}(x, y, z)=\frac{\partial^{2} T_{2}}{\partial x^{2}}+\frac{\partial^{2} T_{2}}{\partial y^{2}}+\frac{\partial^{2} T_{2}}{\partial z^{2}}=0
$$

To evaluate $\nabla^{2} T_{1}(x, y, z)$, it is only necessary to evaluate the second term of $T_{1}(x, y, z)$, which will be denoted $T_{s}(x, y, z)$, since $\nabla^{2} T_{2}(-x, y, z)=0$. This gives

$$
T_{s}(x, y, z)=\frac{S_{0}}{\pi^{2} k_{1}} \frac{1}{\frac{1}{a^{2}}+\frac{1}{b^{2}}+\frac{1}{c^{2}}} \sin \frac{\pi x}{a} \sin \frac{\pi y}{b} \sin \frac{\pi z}{c} .
$$

This gives

$$
\begin{aligned}
& \frac{\partial^{2} T_{s}}{\partial x^{2}}=-\left(\frac{\pi}{a}\right)^{2} \frac{s_{0}}{\pi^{2} K_{1}} \frac{1}{\frac{1}{a^{2}}+\frac{1}{b^{2}}+\frac{1}{c^{2}}} \sin \frac{\pi x}{a} \sin \frac{\pi y}{b} \sin \frac{\pi z}{c}, \\
& \frac{\partial^{2} T}{\partial y^{2}}=-\left(\frac{\pi}{b}\right)^{2} \frac{S_{0}}{\pi^{2} K_{1}} \frac{1}{a^{2}+\frac{1}{b^{2}}+\frac{1}{c^{2}}} \sin \frac{\pi x}{a} \sin \frac{\pi y}{b} \sin \frac{\pi z}{c} \text {, }
\end{aligned}
$$

and 


$$
\frac{\partial^{2} T_{s}}{\partial a^{2}}=-\left(\frac{\pi}{c}\right)^{2} \frac{S_{0}}{\pi^{2} k_{1}} \frac{1}{\frac{1}{a^{2}}+\frac{1}{b^{2}}+\frac{1}{c^{2}}} \sin \frac{\pi x}{a} \sin \frac{\pi y}{b} \sin \frac{\pi z}{c} .
$$

Therefore,

$$
\nabla^{2} T_{s}(x, y, z)=-\frac{S_{0} \sin \frac{\pi x}{a} \sin \frac{\pi y}{b} \sin \frac{\pi z}{c}}{k_{1}},
$$

and

$$
\nabla^{2} T_{1}(x, y, z)=-\frac{S(x, y, z)}{K_{1}} .
$$

The final condition will be checked by evaluating $\left.K_{1} \frac{\partial T}{\partial x}\right|_{x=0}$ and
$\left.K_{2} \frac{\partial T}{\partial x}\right|_{x=0}$ and setting them equal to each other to see if the equality is verified. Performing these operations gives

$$
\begin{aligned}
& K_{2} a \cosh \left[\pi a\left(\frac{1}{b^{2}}+\frac{1}{c^{2}}\right)^{1 / 2}\right] \pi\left(\frac{1}{b^{2}}+\frac{1}{c^{2}}\right)^{1 / 2} \sin \frac{\pi y}{b} \sin \frac{\pi z}{c}= \\
& -k_{1} a \cosh \left[\pi a\left(\frac{1}{b}+\frac{1}{c^{2}}\right)^{1 / 2}\right] \pi\left(\frac{1}{b^{2}}+\frac{1}{c^{2}}\right)^{1 / 2} \sin \frac{\pi y}{b} \sin \frac{\pi z}{c} \\
& +\frac{S_{0}}{\pi^{2}} \frac{1}{\frac{1}{a^{2}}+\frac{1}{b^{2}}+\frac{1}{c^{2}}}\left(\frac{\pi}{a}\right) \sin \frac{\pi y}{b} \sin \frac{\pi z}{c},
\end{aligned}
$$

where

$$
\begin{gathered}
a=\frac{S_{o} a}{\pi^{2}} \cdot \frac{1}{k_{1}+K_{2}} \cdot \frac{1}{1+a^{2}\left(\frac{1}{b^{2}}+\frac{1}{c^{2}}\right)} \cdot \frac{1}{\left(\frac{1}{b^{2}}+\frac{1}{c^{2}}\right)^{1 / 2}} \\
\cdot \frac{1}{\cosh \left[\pi a\left(\frac{1}{b^{2}}+\frac{1}{c^{2}}\right)^{\frac{1}{2}}\right]},
\end{gathered}
$$


or

$$
\frac{S_{0} a}{\pi} \frac{1}{1+a^{2}\left(\frac{1}{b^{2}}+\frac{1}{c^{2}}\right)}=\frac{S_{0}}{a \pi} \frac{1}{\frac{1}{a^{2}}+\frac{1}{b^{2}}+\frac{1}{c^{2}}},
$$

which checks. Therefore the analytical solution to problem 2 is verified.

The analytical solution to problem 3 is

$$
\begin{aligned}
& T_{2}(x, y, z)=-\frac{16 S_{0}}{\pi^{4}\left(k_{1}+K_{2}\right)} \sum_{\text {odd } n}^{\infty} \sum_{\text {odd }}^{\infty}\left(\frac{1}{m n\left(\frac{m^{2}}{b^{2}}+\frac{n^{2}}{c^{2}}\right)} \int_{\sinh \left[\pi a\left(\frac{m^{2}}{b^{2}}+\frac{n^{2}}{c^{2}}\right)\right.}^{\cosh \left[\pi a\left(\frac{m^{2}}{b^{2}}+\frac{n^{2}}{c^{2}}\right)^{1 / 2}-1\right.}\right) \\
& \left.\frac{\sinh \left[\pi(a+x)\left(\frac{m^{2}}{b^{2}}+\frac{n^{2}}{b^{2}}\right)^{1 / 2}\right]}{\cosh \left[\pi a\left(\frac{m_{i}^{2}}{b^{2}}+\frac{n^{2}}{c^{2}}\right)^{1 / 2}\right]} \sin \frac{m \pi y}{b} \sin \frac{n \pi z}{c}\right) \text {, }
\end{aligned}
$$

for $-a \leq x \leq 0,0 \leq y \leq b, 0 \leq z \leq c$, and

$$
\begin{aligned}
T_{1}(x, y, z)=T_{2}(-x, y, z) & +\frac{64 s_{0}}{k_{1} \pi^{5}} \sum_{\ell}^{\infty} \sum_{\text {odd } m}^{\infty} \text { odd } n \sum_{\text {odd }}^{\infty}\left(\frac{1}{\left(\ell m n\left(\frac{l^{2}}{a^{2}}+\frac{m^{2}}{b^{2}}+\frac{n^{2}}{c}\right)\right.}\right. \\
& \left.\cdot \sin \frac{l \pi x}{a} \sin \frac{m \pi y}{b} \sin \frac{n \pi z}{c}\right)
\end{aligned}
$$

for $0 \leq x \leq a, 0 \leq y \leq b$, and $0 \leq z \leq c$.

The solution must satisfy the following: 


$$
\text { (1) } \nabla^{2} T=-\frac{S(x, y, z)}{K} \text {, }
$$

where

$$
S(x, y, z)=S_{0},
$$

for $0 \leq x \leq a, 0 \leq y \leq b, 0 \leq z \leq c$, zero el sewhere,

$$
\text { (2) } T(x, y, z)=0 \text {. }
$$

for all $(x, y, z)$ on outer boundaries, and

$$
\text { (3) } K_{1} \frac{\partial T_{1}}{\partial x}=K_{2} \frac{\partial T}{\partial x} \text {, }
$$

at the interface between the two media.

By inspection it is obvious that $T(x, y, z)=0$ on outer boundaries. To evaluate $\nabla^{2} T_{2}(x, y, z)$ let

$$
\alpha_{m n}=\left(\frac{m^{2}}{b^{2}}+\frac{n^{2}}{c^{2}}\right)^{1 / 2} .
$$

Then

$$
\begin{aligned}
& \frac{\partial^{2} T_{2}}{\partial x^{2}}=\frac{16 s_{0}}{\pi^{2}\left(K_{1}+K_{2}\right)} \sum_{m}^{\infty} \sum_{\text {odd } n}^{\infty} \text { odd }\left(\frac{1}{m n \alpha_{m n}^{2}}\left(\frac{\operatorname{cosin}\left(2 \pi \alpha_{m n}\right)-1}{\sinh \left(a \pi \alpha_{m n}\right)}\right)\right. \\
& \left.\cdot \frac{\sinh \left[\pi(a+x) \alpha_{m n}\right]}{\cosh \left(a \pi a_{m n}\right)} \sin \frac{m \pi y}{b} \sin \frac{n \pi z}{c}\right) \text {, } \\
& \frac{\partial^{2} T_{2}}{\partial y^{2}}=\frac{-16 S_{o}}{\pi^{2}\left(K_{1}+K_{2}\right)} \sum_{m}^{\infty} \sum_{o d d}^{\infty} \sum_{o d d}^{\infty}\left(\frac{m}{n b^{2} \alpha_{m n}^{2}}\left(\frac{\cosh \left(2 \pi \alpha_{m n}\right)-1}{\sinh \left(2 \pi a_{m n}\right)}\right)\right. \\
& \text { - } \left.\frac{\sinh \left[\pi(a+x) a_{m n}\right]}{\cosh \left(a \pi \alpha_{m n}\right)} \sin \frac{m \pi y}{b} \sin \frac{n \pi z}{c}\right) \text {, }
\end{aligned}
$$


and

$$
\begin{aligned}
\frac{\partial^{2} T_{2}}{\partial z^{2}}=\frac{-16 S_{0}}{\pi^{2}\left(K_{1}+K_{2}\right)} & \sum_{\text {odd } n}^{\infty} \sum_{\text {odd }}^{\infty}\left(\frac{n}{m c^{2} \alpha_{m n}^{2}}\left(\frac{\cosh \left(a \pi x_{m n}\right)-1}{\sinh \left(a \pi \alpha_{m}\right)}\right)\right. \\
& \left.\frac{\sinh \left[\pi(a+x) \alpha_{m n}\right]}{\cosh \left(a \pi a_{m n}\right)} \sin \frac{m \pi y}{b} \sin \frac{n \pi z}{c}\right) .
\end{aligned}
$$

Using the definition of $\alpha_{m n}$ gives

$$
\frac{1}{m n}-\frac{m}{n b^{2} \alpha_{m n}^{2}}-\frac{n}{m c^{2} \alpha_{m n}^{2}}=0,
$$

and

$$
\nabla^{2} T_{2}(x, y, z)=0
$$

To evaluate $\nabla^{2} T_{1}(x, y, z)$, it is only necessary to evaluate the second term of $T_{1}(x, y, z)$, which will be denoted $T_{s}(x, y, z)$, since $\nabla^{2} T_{2}(-x, y, z)=0$. This gives

$$
\begin{aligned}
& T_{s}(x, y, z)=\frac{64 S_{0}}{k_{1} \pi^{5}} \sum_{\text {odd } m}^{\infty} \sum_{\text {odd } n}^{\infty} \sum_{\text {odd }}^{\infty} \overbrace{\operatorname{lnn}\left(\frac{\ell^{2}}{a^{2}}+\frac{m^{2}}{b^{2}}+\frac{n^{2}}{c^{2}}\right)} \\
& \text { - } \left.\sin \frac{\ell \pi x}{a} \sin \frac{m \pi y}{b} \sin \frac{n \pi z}{c}\right) \text {. }
\end{aligned}
$$

This gives 


$$
\begin{aligned}
& \frac{\partial^{2} T_{s}}{\partial x^{2}}=-\frac{64 S_{o}}{k_{1} \pi^{3}} \sum_{\ell}^{\infty} \sum_{\text {odd }}^{\infty} \sum_{\text {odd } n}^{\infty} \sum_{\text {odd }}^{\infty}\left(\frac{l^{2} / a^{2}}{l m\left(\frac{l^{2}}{a^{2}}+\frac{n^{2}}{b^{2}}+\frac{n^{2}}{c^{2}}\right)}\right. \\
& \text { - } \left.\sin \frac{\ell \pi x}{a} \sin \frac{m \pi y}{b} \sin \frac{n \pi z}{c}\right) \text {, }
\end{aligned}
$$

and similar expressions for $\frac{\partial^{2} T_{s}}{\partial y^{2}}$ and $\frac{\partial^{2} T s}{\partial z^{2}}$. Combining these gives

$$
\nabla^{2} T_{S}=-\frac{i 4 S_{0}}{K_{1} \pi^{3}} \sum_{\ell}^{\infty} \sum_{\text {odd } m}^{\infty} \sum_{\text {odd } n}^{\infty} \frac{1}{\ell m n} \sin \frac{\ell \pi x}{a} \sin \frac{m \pi y}{b} \sin \frac{n \pi z}{c}
$$

To evaluate this expression, consider a Fourler expansion of the number " 1 " over the region $0 \leq x \leq a, 0 \leq y \leq b, 0 \leq z \leq c$. This gives

$$
1=\sum_{\ell=1}^{\infty} \sum_{m=1}^{\infty} \sum_{n=1}^{\infty} A_{\ell m n} \sin \frac{\ell \pi x}{2} \sin \frac{m \pi y}{b} \sin \frac{n \pi z}{c},
$$

where

$$
A_{\ell m n}=\frac{3}{a b c} \int_{0}^{a} \int_{0}^{b} \int_{0}^{c} \sin \frac{\ell \pi x}{a} \sin \frac{m \pi y}{b} \sin \frac{n \pi z}{c} d z d y d x
$$

Using

$$
\int_{0}^{a} \sin \frac{\ell \pi x}{a} d x=\left(\begin{array}{ll}
\frac{2 a}{\ell \pi} & \ell \text { odd } \\
0 & \ell \text { even }
\end{array}\right.
$$

gives

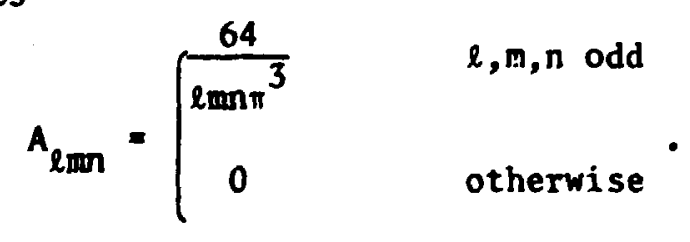


Therefore

$$
\nabla^{2} \mathrm{~T}_{\mathrm{s}}=-\frac{\mathrm{S}_{0}}{\mathrm{~K}_{1}}
$$

The final condition will be checked by evaluating $\left.K_{1} \frac{\partial T_{1}}{\partial x}\right|_{x=0}$ and
$\partial T_{2}$ $\left.K_{2} \quad \frac{\partial T_{2}}{\partial x}\right|_{x=0}$ and setting then equal to each other to see if the equality is verified. Performing these operations gives

$$
\begin{aligned}
& \frac{16 K_{2}}{\pi^{3}\left(K_{1}+K_{2}\right)} \sum_{m}^{\infty} \sum_{\text {odd } n}^{\infty} \frac{1}{\text { odd }}\left(\frac{\cosh \left(a \pi a_{m n}\right)-1}{\operatorname{minh}\left(a \pi \alpha_{m n}\right)}\right) \sin \frac{m \pi y}{b} \sin \frac{n \pi z}{c} \\
& =\frac{-16 K_{1}}{\pi_{i}^{3}\left(K_{1}+K_{2}\right)} \sum_{\text {odd } n}^{\infty} \sum_{\text {odd }}^{\infty} \frac{1}{m n \alpha_{m n}}\left(\frac{\cosh \left(a \pi \alpha_{m n}\right)-1}{\sinh \left(a \pi \alpha_{m n}\right)}\right) \sin \frac{m \pi y}{b} \sin \frac{n \pi z}{c} \\
& +\frac{64}{a \pi} \sum_{\ell}^{\infty} \sum_{\text {odd } m}^{\infty} \sum_{\text {odd } n}^{\infty} \frac{1}{m n\left(\frac{l^{2}}{a^{2}}+\frac{m^{2}}{b^{2}}+\frac{n^{2}}{c^{2}}\right)} \sin \frac{m \pi y}{b} \sin \frac{n \pi z}{c} .
\end{aligned}
$$

To verify this equation, it is necessary to verify that

$$
\frac{\pi}{4 B} \frac{\operatorname{oosh} \pi \beta-1}{\sinh \pi \beta}=\sum_{\ell \text { odd }}^{\infty} \frac{1}{\ell^{2}+\beta^{2}},
$$

where

$$
\beta=a \alpha_{\mathrm{mn}} \text {. }
$$

To verify Eq. $(A-42)$, consider a Fourler cosine expansion of $\frac{\cosh \beta(\pi-x)}{B \sinh \beta \pi}$. Using 


$$
\int_{0}^{\pi} \frac{\cosh B(\pi-x)}{\beta \sinh B \pi} \cos \ell x d x=\frac{1}{\ell^{2}+B^{2}},
$$

gives

$$
\frac{\cosh \beta(\pi-x)}{\beta \sin \beta \pi}=\frac{1}{\pi \beta^{2}}+\sum_{\ell=1}^{\infty} \frac{2}{\pi} \frac{1}{\ell^{2}+\beta^{2}} \cos \ell x .
$$

Setting $x=0$ gives

$$
\frac{\cosh \beta \pi}{\beta \sin \beta \pi}=\frac{1}{\pi \beta^{2}}+\sum_{\ell=1}^{\infty} \frac{2}{\pi} \frac{1}{\ell^{2}+\beta^{2}}
$$

Setting $x=\pi$ gives

$$
\frac{1}{\beta \sinh \beta \pi}=\frac{1}{\pi \beta^{2}}-\sum_{\ell=1}^{\infty} \frac{2}{\pi} \frac{(-1)^{\ell}}{\ell^{2}+\beta^{2}} .
$$

Subtracting Eq. (A-47) from Eq. (A-46) and multiplying by $\frac{\pi}{4}$ gives

$$
\frac{\pi}{4 \beta} \frac{\cosh \pi \beta-1}{\sinh \pi \beta}=\sum_{\ell \text { odd }}^{\infty} \frac{1}{\ell^{2}+\beta^{2}},
$$

which verifies the equality. Therefore the analytical solution to problem 3 is verified.

The analytical solution to problem 4 is

$$
T_{2}(x, y, z)=\frac{K_{1}}{k_{1}+K_{2}} \frac{\operatorname{sinin}\left[\pi(a+x)\left(\frac{1}{2}+\frac{1}{c^{2}}\right)^{1 / 2}\right] \sin \frac{\pi y}{b} \sin \frac{\pi z}{c}}{b^{2}\left(\pi a\left(\frac{1}{b^{2}}+\frac{1}{c^{2}}\right)^{1 / 2}\right] \cosh \left[\pi a\left(\frac{1}{b^{2}}+\frac{1}{c^{2}}\right)^{1 / 2}\right]}
$$

for $-a \leq x \leq 0,0 \leq y \leq b, 0 \leq z \leq c$, and 


$$
T_{1}(x ; y, z)=T_{2}(-x, y, z)+\frac{\sinh \left[\pi \times\left(\frac{1}{b^{2}}+\frac{1}{c^{2}}\right)^{1 / 2}\right]}{\sinh \left[\pi a\left(\frac{1}{b^{2}}+\frac{1}{c^{2}}\right)^{1 / 2}\right]} \sin \frac{\pi y}{b} \sin \frac{\pi z}{c} \quad(A-50)
$$

for $0 \leq x \leq a, 0 \leq y \leq b$, and $0 \leq z \leq c$.

The solution must satisfy the following:

(1) $\nabla^{2} \mathrm{~T}=0$,

(2) $T(x, y, z)= \begin{cases}\sin \frac{\pi y}{b} \sin \frac{\pi z}{c}, & \text { on surface at } x=a, \\ 0 \quad & \begin{array}{l}\text { on all other external } \\ \text { boundaries }\end{array}\end{cases}$

and

(3) $K_{1} \frac{\partial T_{1}}{\partial x}=\frac{\partial T_{2}}{\partial x}$,

at the interface between the two media.

By inspection it is obvious that the external boundary conditions as given in EG. $(A-52)$ are met. To evaluate $\nabla^{2} I_{2}(x, y, z)$ let

$$
T_{2}(x, y, z)=\alpha \sinh \left[\pi(a+x)\left(\frac{1}{b^{2}}+\frac{1}{c^{2}}\right)^{1 / 2}\right] \sin \frac{\pi y}{b} \sin \frac{\pi z}{c},
$$

then from Eqs. $(A-7)$ and $(A-11)$

$$
\nabla^{2} \mathrm{~T}_{2}(x, y, z)=0
$$

To evaluate $\nabla^{2} T_{1}(x, y, z)$, it is only necessary to evaluate the second term of $T_{1}(x, y, z)$ which will be denoted $T_{s}(x, y, z)$. This gives

$$
T_{s}(x, y, z)=B \sinh \left[\pi x\left(\frac{1}{b}+\frac{1}{c^{2}}\right)^{1 / 2}\right] \sin \frac{\pi y}{b} \sin \frac{\pi L}{c} .
$$


Therefore

$$
\begin{aligned}
& \frac{\partial^{2} T_{s}}{\partial x^{2}}=B \pi^{2}\left(\frac{1}{b^{2}}+\frac{1}{c^{2}}\right) \sinh \left[\pi x\left(\frac{1}{b^{2}}+\frac{1}{c^{2}}\right)^{1 / 2}\right] \sin \frac{\pi y}{b} \sin \frac{\pi z}{c}, \\
& \frac{\partial^{2} T_{s}}{\partial y^{2}}=-\beta \frac{\pi^{2}}{b^{2}} \sinh \left[\pi x\left(\frac{1}{b^{2}}+\frac{1}{c^{2}}\right)^{1 / 2}\right] \sin \frac{\pi y}{b} \sin \frac{\pi z}{c},
\end{aligned}
$$

and

$$
\frac{\partial^{2} T_{s}}{\partial z^{2}}=-B \frac{\pi^{2}}{c^{2}} \sinh \left[\pi \times\left(\frac{1}{b}+\frac{1}{c^{2}}\right)^{1 / 2}\right] \sin \frac{\pi y}{b} \sin \frac{\pi z}{c}
$$

This gives

$$
\nabla^{2} T_{1}(x, y, z)=0
$$

The final condition will be checked by evaluating $\left.k_{1} \frac{\partial T}{\partial x}\right|_{x=0}$ and $\left.K_{2} \frac{\partial T}{\partial x}\right|_{x=0}$ and setting them equal to each other to see if the equality is verified. Performing these operations gives

$$
\begin{aligned}
& k_{2} k_{1} \pi\left(\frac{1}{b^{2}}+\frac{1}{c^{2}}\right)^{1 / 2} \sin \frac{\pi y}{b} \sin \frac{\pi z}{c}= \\
& \overline{K_{1}+K_{2}} \frac{\sinh \left[\pi a\left(\frac{1}{b^{2}}+\frac{1}{c^{2}}\right)^{1 / 2}\right]}{} \\
& -\frac{k_{1}{ }^{2}}{k_{1}+K_{2}} \frac{\pi\left(\frac{1}{b^{2}}+\frac{1}{c^{2}}\right)^{1 / ?} \sin \frac{\pi y}{b} \sin \frac{\pi z}{c}}{\sinh \left[\pi a\left(\frac{1}{b^{2}}+\frac{1}{c^{2}}\right)^{1 / 2}\right]} \\
& +\frac{k_{1} \pi\left(\frac{1}{b^{2}}+\frac{1}{c^{2}}\right)^{1 / 2} \sin \frac{\pi y}{b} \sin \frac{\pi z}{c}}{\sinh \left[\pi a\left(\frac{1}{b^{2}}+\frac{1}{c^{2}}\right)^{1 / 2}\right]},
\end{aligned}
$$


which checks. Therefore the analytical solution to problem 4 is verified.

The analytical solution to problem 5 is

$$
\begin{aligned}
T_{2}(x, y, z)= & \frac{S_{0} a}{\pi^{2}} \frac{1}{\left(\frac{1}{b^{2}}+\frac{1}{c^{2}}\right)^{1 / 2}} \frac{1}{1+a^{2}\left(\frac{1}{b^{2}}+\frac{1}{c^{2}}\right)} \\
& \frac{\left(K_{1}+K_{3}\right) \cosh \left[\pi a\left(-\frac{1}{b^{2}}+\frac{1}{c^{2}}\right)^{1 / 2}\right]+K_{1}}{\left.K_{1}+K_{3}\right)\left(K_{1}+K_{2}\right) \cosh ^{2}\left[\pi a\left(\frac{1}{b^{2}}+\frac{1}{c^{2}}\right)^{1 / 2}\right]-K_{1}^{2}} \\
& \cdot \sinh \left[\pi(a+x)\left(\frac{1}{b^{2}}+\frac{1}{c^{2}}\right)^{1 / 2}\right] \sin \frac{\pi y}{b} \sin \frac{\pi z}{c},
\end{aligned}
$$

for $-a \leq x \leq 0,0 \leq y \leq b, 0 \leq z \leq c$,

$$
\begin{aligned}
T_{3}(x, y, z)= & \frac{S_{0} a}{\pi^{2}} \frac{1}{\left(\frac{1}{b^{2}}+\frac{1}{c^{2}}\right) 1 / 2} \frac{1}{1+a^{2}\left(\frac{1}{b^{2}}+\frac{1}{c^{2}}\right)} \\
& \frac{\left(K_{1}+K_{2}\right) \cosh \left[\pi a\left(\frac{1}{b^{2}}+\frac{1}{c^{2}}\right)^{1 / 2}\right]+K_{1}}{\left(K_{1}+K_{3}\right)\left(K_{1}+K_{2}\right) \cosh ^{2}\left[\pi a\left(\frac{1}{b^{2}}+\frac{1}{c^{2}}\right)^{1 / 2}\right]-K_{1}^{2}} \\
& \cdot \sinh \left[\pi(2 a-x)\left(\frac{1}{b^{2}}+\frac{1}{2}\right)^{1 / 2}\right] \sin \frac{\pi y}{b} \sin \frac{\pi z}{c},
\end{aligned}
$$

for $a \leq x \leq 2 a, 0 \leq . \leq b, 0 \leq z \leq c$,

and 


$$
\begin{aligned}
T_{1}(x, y, z)= & T_{2}(-x, y, z)+T_{3}(2 a-x, y, z) \\
& +\frac{s_{0}}{\pi^{2} K_{1}} \frac{1}{\frac{1}{a^{2}}+\frac{1}{b^{2}}+\frac{1}{c^{2}}} \sin \frac{\pi x}{a} \sin \frac{\pi y}{b} \sin \frac{\pi z}{c},
\end{aligned}
$$

for $0 \leq x \leq a, 0 \leq y \leq b$, and $0 \leq z \leq c$.

The solution must satisfy the following:

$$
\text { (1) } \nabla^{2} T=-\frac{S(x, y, z)}{K} \text {, }
$$

where

$$
S(x, y, z)=S_{c} \sin \frac{\pi x}{a} \sin \frac{\pi y}{b} \sin \frac{\pi z}{c}
$$

for $0 \leq x \leq a, 0 \leq y \leq b, 0 \leq z \leq c$, and zero el sewhere,

(2) $T(x, y, z)=0$,

for all $(x, y, z)$ on outer boundaries,

(3) $K_{1} \frac{\partial T_{1}}{\partial x}=K_{2} \frac{\partial T_{2}}{\partial x}$,

at the $x=0$ interface, and

(4) $K_{1} \frac{\partial T_{1}}{\partial x}=K_{3} \frac{\partial T_{3}}{\partial x}$,

at the $x=a$ interface.

By inspection it is obvious that $T(x, y, z)=0$ on the outer houndaries. From the verification of problem 2 it follows that Eq. (A-65) is also verified. The condition at the $x=0$ interface will be checked by evaluating $\left.K_{1} \frac{\partial T}{\partial x}\right|_{x=0}$ and $\left.K_{2} \frac{\partial T}{\partial x}\right|_{\lambda=0}$ and setting them 
equal to each other to see if the equality is verified. Perforaing these operations gives

$$
\begin{gathered}
\frac{\left(K_{1}+K_{2}\right) \cosh \left[\pi a\left(\frac{1}{b^{2}}+\frac{1}{c^{2}}\right)^{1 / 2}\right]\left(\left(K_{1}+K_{3}\right) \cosh \left[\pi a\left(\frac{1}{b^{2}}+\frac{1}{c^{2}}\right)^{1 / 2}+K_{1}\right)\right.}{\left(K_{1}+K_{3}\right)\left(K_{1}+K_{2}\right) \cosh ^{2}\left[\pi a\left(\frac{1}{b^{2}}+\frac{1}{c^{2}}\right)^{1 / 2}\right]-K_{1}^{2}} \\
=\frac{K_{1}\left(K_{1}+K_{2}\right) \cosh \left[\pi a\left(\frac{1}{b^{2}}+\frac{1}{c^{2}}\right)^{1 / 2}\right]+K_{1}^{2}}{\left(K_{1}+K_{3}\right)\left(K_{1}+K_{2}\right) \cosh ^{2}\left[\pi a\left(\frac{1}{b^{2}}+\frac{1}{c^{2}}\right)^{1 / 2}\right]-K_{1}^{2}},
\end{gathered}
$$

which checks. The final condition at the $x=a$ interface will be checked by evaluating $\left.K_{1} \frac{\partial T}{\partial x}\right|_{x=a}$ and $\left.K_{3} \frac{\partial T}{\partial x}\right|_{x=a}$ and setting them equal to each other to see if the equality is verified. Performing these operations gives

$$
\begin{gathered}
\frac{\left(K_{1}+K_{3}\right) \cosh \left[\pi a\left(\frac{1}{b^{2}}+\frac{1}{c^{2}}\right)^{1 / 2}\right]\left(\left(K_{1}+K_{2}\right) \cosh \left[\pi a\left(-\frac{1}{2}+\frac{1}{c^{2}}\right)^{1 / 2}\right]+K_{1}\right)}{\left(K_{1}+K_{3}\right)\left(K_{1}+K_{2}\right) \cosh ^{2}\left[\pi a\left(\frac{1}{b^{2}}+\frac{1}{c^{2}}\right)^{1 / 2}\right]-K_{1}^{2}} \\
=\frac{K_{1}\left(K_{1}+K_{3}\right) \cosh \left[\pi a\left(\frac{1}{b^{2}}+\frac{1}{c^{2}}\right)^{1 / 2}\right]+K_{1}^{2}}{\left(K_{1}+K_{3}\right)\left(K_{1}+K_{2}\right) \cosh ^{2}\left[\pi a\left(\frac{1}{b^{2}}+\frac{1}{c^{2}}\right)^{1 / 2}\right]-K_{1}^{2}+1,}
\end{gathered}
$$

which checks. Therefore the analytical solution to problem 5 is verified.

The analytical solution to problem 6 is 


$$
T_{2}(r, z)=\frac{S_{0} \pi l}{\pi^{2}+l^{2} \alpha_{1}{ }^{2}} \frac{J_{0}\left(a_{1} r\right)}{a_{1}\left(K_{1}+K_{2}\right)} \frac{\sinh a_{1}(l+z)}{\cos . a_{1} l},
$$

for $0 \leq r \leq a,-i \leq z \leq 0$,

and

$$
T_{1}(r, z)=T_{2}(r,-z)+\frac{S_{0}}{K_{1}\left(a_{1}^{2}+\frac{\pi^{2}}{l^{2}}\right)} J_{0}\left(a_{1} r\right) \sin \frac{\pi z}{l},
$$

for $0 \leq r \leq a$, and $0 \leq z \leq l$.

The solution must satisfy the following:

$$
\text { (1) } \nabla^{2} T=-\frac{S(r, z)}{K} \text {, }
$$

where

$$
S(r, z)=S_{0} J_{0}\left(\alpha_{1} r\right) \sin \frac{\pi z}{l},
$$

for $0 \leq \mathbf{r} \leq \mathrm{a}, 0 \leq: \leq \ell$, and zero el sewhere,

(2) $T(r, z)=0$,

for all $(r, z)$ on outer boundaries, and

(3) $K_{1} \frac{\partial T_{1}}{\partial z}=K_{2} \frac{\partial T_{2}}{\partial z}$,

at the interface between the two media.

By inspection and from the definition of $\alpha_{1}$ as the first zero of $J_{0}$ (aa), it is obvious that $T(r, z)=0$ on the outer boundaries. 
For cylindrical geometries with anguiar symmetry

$$
\nabla^{2}=\frac{1}{r} \frac{\partial}{\partial r}\left(r \frac{\partial}{\partial r}\right)+\frac{\partial^{2}}{\partial z^{2}} .
$$

Using

$$
\frac{1}{r} \frac{\partial}{\partial r}\left(r \frac{\partial J_{0}\left(a_{1} r\right)}{\partial r}\right)=-a_{1}^{2} J_{0}\left(\alpha_{1} r\right),
$$

and

$$
\frac{\partial^{2}}{\partial z^{2}} \sinh a_{1}(l+z)=a_{1}^{2} \sinh a_{1}(l+z),
$$

gives

$$
\nabla T_{2}(r, 2)=0
$$

To evaluate $\nabla^{2} T_{1}(r, z)$, it is only necessary to evaluate the second term of $T_{1}(r, z)$, which will be denoted $T_{s}(r, x)$. This gives

$$
\nabla T_{s}(r, z)=-\frac{s_{0} J_{0}\left(a_{1} r\right) \sin \pi_{\ell} z}{x_{1}}
$$

Therefore

$$
\nabla^{2} T_{1}(r, z)=-\frac{S(r, z)}{K_{1}}
$$

The final condition will be checked by evaluating $\left.K_{1} \frac{\partial T_{1}}{\partial z}\right|_{z=0}$ and $\left.K_{2} \frac{\partial T_{2}}{\partial z}\right|_{z=0}$ and setting them equal to each other to see if the equality is verified. Performing these operations gives 


$$
\begin{aligned}
\frac{S_{0} \pi \ell}{\pi^{2}+\ell^{2} a_{1}^{2}} \frac{K_{2}}{K_{1}+K_{2}} J_{0}\left(a_{1} r\right)= & -\frac{s_{0} \pi \ell}{\pi^{2}+\ell^{2} a_{1}^{2}} \frac{K_{1}}{K_{1}+K_{2}} J_{0}\left(a_{1} r\right) \\
& +\frac{s_{0} \pi}{\ell\left(a_{1}^{2}+\frac{\pi^{2}}{\ell^{2}}\right)} J_{0}\left(a_{1} r\right)
\end{aligned}
$$

which checks. Therefore the analytical solution to problem 6 is verified.

The analytical solution to problem 7 is

$$
T_{2}(r, z)=\frac{2 S_{0}}{a\left(K_{1}+K_{2}\right)} \sum_{n=1}^{\infty}\left(\frac{\cosh \alpha_{n} \ell-1}{a_{n}^{3} J_{1}\left(\alpha_{n} a\right)} \frac{J_{0}\left(\alpha_{n} r\right) \sinh a_{n}(l+z)}{\sinh \alpha_{n} l \cosh \alpha_{n} l}\right)
$$

for $0 \leq \mathrm{r} \leq \mathrm{a},-\ell \leq \mathrm{z} \leq 0$

and

$$
T_{1}(r, z)=T_{2}(r,-z)+\frac{8 S_{0}}{a \pi} \sum_{m \text { odd }} \frac{1}{m} \sin \frac{m \pi z}{l} \sum_{n=1}^{\infty} \frac{J_{o}\left(a_{n} r\right)}{K_{1} \alpha_{n}\left(\frac{m^{2} \pi^{2}}{l^{2}}+\alpha_{n}^{2}\right) J_{1}\left(\alpha_{n} a\right)}
$$

for $0 \leq \mathbf{r} \leq \mathbf{a}$, and $0 \leq z \leq \ell$

The solution must satisfy the following

$$
\text { (1) } \nabla^{2} T=-\frac{s(r, z)}{K}
$$

where

$$
S(r, z)=S_{0}
$$

for $0 \leq \mathbf{r} \leq \mathrm{a}, 0 \leq z \leq \ell$, and zero elsewhere 
(2) $T(r, z)=0$

for ali $(r, z)$ on outer boundaries, and

(3) $K_{1} \frac{\partial T_{1}}{\partial z}=K_{2} \frac{\partial T_{2}}{\partial z}$

at the interface $t$ : tween the two media.

By inspection and from the definition of the $\alpha_{n}$ as the zeroes of $\mathrm{J}_{0}(a a)$, it is obvious that $\mathrm{T}(\mathrm{r}, \mathrm{z})=0$ on outer boundaries. Using

$$
\frac{1}{r} \frac{\partial}{\partial r}\left(r \frac{\partial J_{0}\left(\alpha_{n} r\right)}{\partial r}\right)=-\alpha_{n}^{2} J_{0}\left(\alpha_{n} r\right)
$$

and

$$
\frac{\partial^{2} \sinh \alpha_{n}(l+z)}{\partial z^{2}}=\alpha_{n}^{2} \sinh \alpha_{n}(l+z)
$$

gives

$$
\nabla^{2} \mathrm{~T}_{2}(\mathrm{r}, z)=0
$$

To evaluate $\nabla^{2} T_{1}(r, z)$, it is only necessary to evaluate the second term of $T_{1}(r, z)$ which will be denoted $T_{S}(r, z)$. Using

$$
\frac{\partial^{2}}{\partial z^{2}} \sin \frac{m \pi z}{l}=-\frac{m^{2} \pi^{2}}{l^{2}} \sin \frac{m \pi z}{l}
$$

gives

$$
\nabla^{2} T_{s}(r, z)=-\frac{8 S_{0}}{a T} \sum_{\text {odd }}^{\infty} \sum_{n=1}^{\infty} \frac{J_{0}\left(\omega_{n} r\right)}{m K_{1} \alpha_{n} J_{1}\left(\alpha_{n} a\right)} \sin \frac{m \pi z}{\ell} .
$$

To evaluate this, consider a series expansion of the number " 1 " over the region $0 \leq r \leq a, 0 \leq z \leq \ell$. This is given by 


$$
1=\sum_{m=1}^{\infty} \sum_{n=1}^{\infty} A_{\operatorname{mn}} \sin \frac{m \pi z}{\ell} J_{0}\left(\alpha_{n} r\right)
$$

where

$$
A_{\operatorname{mn}}=\frac{4}{a^{2} \ell J_{1}^{2}\left(\alpha_{n} a\right)} \int_{0}^{a} \int_{0}^{l} \sin \frac{m m z}{l} J_{0}\left(\alpha_{n} r\right) r d z d r .
$$

Using

$$
\int_{0}^{\ell} \sin \frac{m \pi z}{\ell} d z=\left(\begin{array}{cc}
\frac{2 l}{m \pi} & m \text { odd } \\
0 & \text { m even }
\end{array}\right.
$$

and

$$
\int_{0}^{a} r J_{0}\left(\alpha_{n} r\right) d r=\frac{a}{\alpha_{n}} J_{1}\left(\alpha_{n} a\right)
$$

gives

$$
A_{m n}=\frac{8}{m \pi a J_{1}\left(\alpha_{n} a\right)}
$$

Therefore

$$
\nabla^{2} \mathrm{~T}_{\mathrm{s}}(\mathrm{r}, \mathrm{z})=-\frac{\mathrm{s}_{\mathrm{o}}}{\mathrm{K}_{1}}
$$

The final condition will be checked by evaluating $\left.K_{1} \frac{\partial T}{\partial z}\right|_{z=0}$ and
$\left.K_{2} \frac{\partial T_{2}}{\partial z}\right|_{z=0}$ and setting them equal to each other to see if the equality
is verified. Performing these operations gives 


$$
\begin{aligned}
& \frac{2 S_{0} K_{2}}{a\left(K_{1}+K_{2}\right)} \sum_{n=1}^{\infty} \frac{\cosh a_{n} \ell-1}{a_{:}^{2} J_{1}\left(a_{n} a\right)} \frac{J_{0}\left(a_{n} r\right)}{\sinh \alpha_{n}^{\ell}} \\
& =-\frac{2 S_{0} K_{1}}{a\left(K_{1}+K_{2}\right)} \sum_{n=1}^{\infty} \frac{\cosh \alpha_{n}-1}{a_{n}^{2} J_{1}\left(\alpha_{n} a\right)} \frac{J_{0}\left(\alpha_{n} r\right)}{\sin \alpha_{n}^{l}} \\
& +\frac{8 S_{0}}{a l} \sum_{m \text { odd }}^{\infty} \sum_{n=1}^{\infty} \frac{J_{o}\left(a_{n} r\right)}{a_{n}\left(\frac{m^{2} \pi^{2}}{l^{2}}+a_{n}^{2}\right) J_{1}\left(a_{n} a\right)},
\end{aligned}
$$

or

$$
\begin{aligned}
\frac{l}{4} \sum_{n=1}^{\infty} \frac{\cosh \alpha_{n} l-1}{a_{n}^{2} J_{1}\left(a_{n} a\right)} \frac{J_{0}\left(\alpha_{n} r\right)}{\sinh \alpha_{n}^{l}} \\
\quad=\sum_{m \text { odd } n=1}^{\infty} \sum_{\alpha_{n}\left(\frac{m^{2} \pi^{2}}{l^{2}}+a_{n}^{2}\right) J_{1}\left(a_{n} a\right)}^{J_{o}\left(\alpha_{n} r\right)}
\end{aligned}
$$

To verify this equality, it is necessary to verify that

$$
\frac{\ell}{4 a_{n}} \frac{\cosh a_{n} \ell-1}{\sinh a_{n} \ell}=\sum_{\operatorname{codd}}^{\infty} \frac{1}{\frac{m^{2} \pi^{2}}{l^{2}}+a_{n}^{2}}
$$

or, letting $\beta=\frac{\ell \alpha_{n}}{\pi}$,

$$
\frac{\pi}{4 \beta}\left(\frac{\cosh \pi \beta-i}{\sinh \pi \beta}\right)=\sum_{m \text { odd }}^{\infty} \frac{1}{m^{2}+\beta^{2}} .
$$

But, this is precisely Eq. (A-42) which was verified in checking the 
solution to problem 3. Therefos the analyisal solution to problem 7 is verified.

The analytical solution to problem 8 is

$$
T_{2}(r, z)=\frac{K_{1}}{K_{1}+K_{2}} J_{0}\left(\alpha_{1} r\right) \frac{\sinh a_{1}(l+2)}{\sinh \alpha_{1} l \cosh a_{1} l}
$$

for $0 \leq \mathbf{r} \leq \mathrm{a},-\boldsymbol{l} \leq \mathbf{2} \leq 0$,

and

$$
T_{1}(r, z)=T_{2}(r,-z)+J_{0}\left(\alpha_{1} r\right) \frac{\sinh \alpha_{1} z}{\sinh \alpha_{1} l}
$$

for $0 \leq \mathbf{r} \leq \mathbf{a}$, and $0 \leq z \leq \ell$.

The solution must satisfy the following:
(1) $\nabla^{2} T=0$
(2) $T(r, z)= \begin{cases}J_{0}\left(\alpha_{1} r\right), & \text { on the surface at } z=l \\ 0, & \text { or. all other external boundaries }\end{cases}$

and

(3) $K_{1} \frac{\partial T_{1}}{\partial z}=K_{2} \frac{\partial T_{2}}{\partial z}$,

at the interface between the two media.

By inspection it is obvious that the external boundary conditions as given in Eq. (A-109) are met. Using

$$
\frac{1}{r} \frac{\partial}{\partial r}\left(r \frac{\partial}{\partial r} J_{0}\left(a_{1} r\right)\right)=-a_{1}^{2} J_{0}\left(\alpha_{1} r\right)
$$


145

and

$$
\frac{\partial^{2}}{\partial z^{2}} \sinh \alpha_{1}(\ell+z)=\alpha_{1}^{2} \sinh \alpha_{1}(\ell+z)
$$

gives

$$
\nabla^{2} \mathrm{~T}=0
$$

The final condition will be checked by evaluating $\left.K_{1} \frac{\partial T}{\partial z}\right|_{z=0}$ and $\left.K_{2} \frac{\partial T}{\partial z}\right|_{z=0}$ and setting them equal to each other to see if the equality is verified. Performing these operations gives

$$
\frac{K_{1} K_{2}}{K_{1}+K_{2}} \alpha_{1} \frac{J_{0}\left(\alpha_{1} r\right)}{\sinh \alpha_{1} l}=-\frac{K_{1}^{2}}{K_{1}: K_{2}} \alpha_{1} \frac{J_{0}\left(\alpha_{1} r\right)}{\sinh \alpha_{1} l}+K_{1} \alpha_{1} \frac{J_{0}\left(\alpha_{1} r\right)}{\sinh \alpha_{1}^{l}}, \quad(A-114)
$$

which checks. Therefore the analytical solution to problem 8 is verified. 


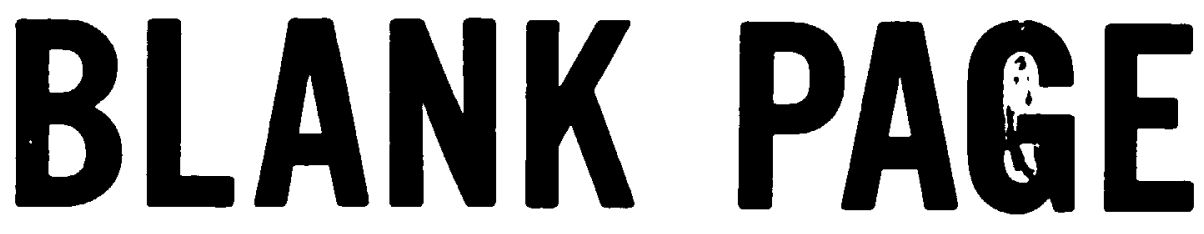




\section{APPENDIX B}

\section{DERIVATION OF A BOUNDED NEXT EVENT ESTIMATOR}

The following derivation will be made assuming a monoenergetic flux and isotropic scattering. These assumptions are made for simplicity of notation only. The results are not changed if the flux is assumed to be energy dependent and if the scattering is anisotropic.

The flux at a point $r=0$ can be represented by

$$
\phi(r=0)=\int_{r} \Sigma_{S} \Phi_{a v g}(r) 4 \pi r^{2} \frac{e^{-\Sigma r}}{4 \pi r^{2}} d r+\int_{r} S(r) 4 \pi r^{2} \frac{e^{-\sum r}}{4 \pi r^{2}} d r
$$

where

$$
\phi_{\operatorname{avg}}(r)=\frac{\iint_{A} \phi d A}{\iint_{A} d A}
$$

and $A$ is the surface area at $r$.

The standard Monte Carl" unbounded next event estimator uses each collision as an estimate of $\Sigma_{t^{\phi}}$ avg $(r) 4 \pi r^{2}$ and scores $\frac{\Sigma_{s} e^{-\Sigma \tau}}{\Sigma_{t} 4 \pi r^{2}}$ at each collision site. The estimator is considered unbounded since the score is unbounded as $r$ approaches zero.

This problem can be eliminated by scoring all collisions within a sphere of radius "a" by $\frac{e^{-\sum r}}{4 \pi r^{2}}$ where $d$ is calculated from

$$
\int_{0}^{a} \Sigma_{s} \Phi_{a v g}(r) 4 \pi r^{2} \frac{e^{-\Sigma r}}{4 \pi d^{2}} d r=\int_{0}^{a} \Sigma_{s} \Phi_{a v g}(r) 4 \pi r^{2} \frac{e^{-\Sigma r}}{4 \pi r^{2}} d r
$$


This calculation depends upon the flux shape assuned for $\phi(r)$. In this appendix "d" will be calculated as a function of "a" for (1) a flat flux and (2) an exponentially decreasing flux.

$$
\begin{aligned}
& \text { Assume } \phi(r)=\phi_{0}, \text { a constant. Then } \phi_{a v g}(r)=\phi_{0} \text { and } \\
& d^{2}=\frac{\int_{0}^{a} r^{2} e^{-\Sigma r} d r}{\int_{0}^{a} e^{-\Sigma r} d r} .
\end{aligned}
$$

Evaluating the above integrals gives

$$
d^{2}=a^{2}\left(\frac{2}{\lambda^{2}}-\frac{e^{-\lambda}\left(1+\frac{2}{\lambda}\right)}{1-e^{-\lambda}}\right)
$$

where $\lambda=\sum a$ is the radius of the bounding sphere in mean free paths.

This can also be written in series form as

$$
d^{2}=a^{2} \frac{\sum_{n=0}^{\infty}(-1)^{n} \frac{(n+1)(n+2)}{(n+3) !} \lambda^{n}}{\sum_{n=0}^{\infty}(-1)^{n} \frac{\lambda^{n}}{(n+1) !}}
$$

Next assume $\phi=\phi_{0} e^{-\Sigma x} \cdot \phi_{a v g}(r)$ is then given by

$$
\phi_{\operatorname{avg}}(r)=\frac{1}{4 \pi r^{2}} \iint_{A} \phi_{0} e^{-\Sigma x} d A
$$

where $A$ is the area of the sphere with radius $r$ and $I=\left(x^{2}+y^{2}+z^{2}\right)^{1 / 2}$. Evaluating this integral gives 


$$
\phi_{\operatorname{avg}}(r)=\phi_{0} \frac{\sinh \left(\Sigma_{r}\right)}{\Sigma r},
$$

and

$$
d^{2}=\frac{\int_{0}^{a} r^{2} \frac{\sinh \left(\Sigma_{r}\right)}{\Sigma r} e^{-\Sigma r} d r}{\int_{0}^{a} \frac{\sinh \left(\Sigma_{r}\right)}{\Sigma r} e^{-\Sigma r} d r}
$$

Evaluating these integrals gives

$$
d^{2}=a^{2}\left(\frac{\frac{1}{2}-\frac{1}{c^{2}+\frac{e^{-c}}{c^{2}}(1+c)}}{E i(c)+\ln (c)+\gamma}\right)
$$

where $c=2 \Sigma a$,

$Y$ is Euler's constant, and

$E_{1}(c)$ is the exponential integral function of $c$.

This can also be written in series form as:

$$
d^{2}=a^{2} \frac{\sum_{n=0}^{\infty}(-1)^{n} \frac{(n+2) c^{n}}{(n+3) !}}{\sum_{n=0}^{\infty}(-1)^{n} \frac{c^{n}}{(n+1)(n+1) !}}
$$

Table 20 gives $d^{2} / a^{2}$ versus $\Sigma_{a}$ for both assumptions, i.e., $\phi=\phi_{0}$ and $\phi=\phi_{0} e^{-\sum x}$.

Note that for bounding spheres with radii up to one mean path in length the flux shape used has very little effect on $d^{2}$, and therefore on the score used in the estimator. In estimating the flux at a point 
wich is not near a strong point source or an external boundary, the above nethod should therefore be applicable for bounding spheres up to one mean free path in radius since the flux shape will usually be somewhere between 1 constant and exponentially decaying.

TABLE 20

$$
\mathrm{d}^{2} / \mathrm{a}^{2} \text { VERSUS } \mathrm{a}
$$

\begin{tabular}{ccc}
\hline$a$ & & $\mathrm{~d}^{2} / \mathrm{a}^{2}$ \\
\cline { 2 - 3 }$(\operatorname{mfp})$ & for $\phi=\phi_{0}$ & for $\phi=\phi_{0} \mathrm{e}^{-\sum \mathrm{x}}$ \\
\hline .1 & .3250 & .3252 \\
.25 & .3127 & .3136 \\
.50 & .2925 & .2960 \\
1.00 & .2541 & .2665 \\
\hline
\end{tabular}

This procedure does not require a modification of the randon walk procedure and may be implemented very easily into Monte Carlo programs which use next event estimators. 
APPENDIX C

\section{LISTING OF COMPUTER INPUT DATA AND SELECTED OUTPUT}

The input to MORSE-SGC and its output are given on the following pages for the coupled gamma radiation and heat conduction problem solved in Chapter VI.

The listings of the analysis routines are given on the next three pages. The input data to LAVA to generate the cross sections and to MORSE-SGC is given on the following page, and the remaining pages in this Appendix are MORSE-SGC output. 


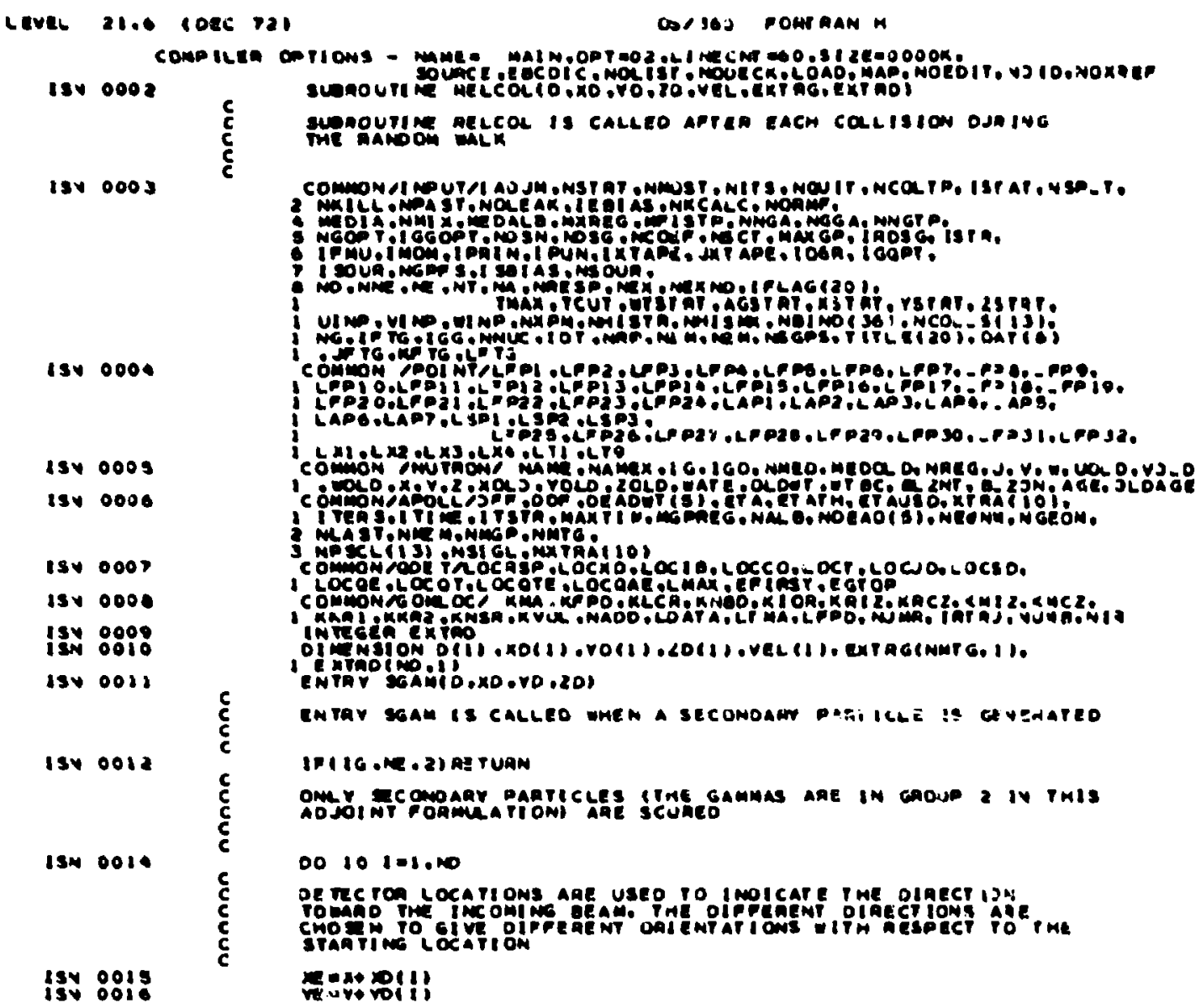

184 0002

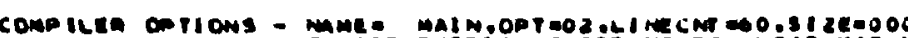

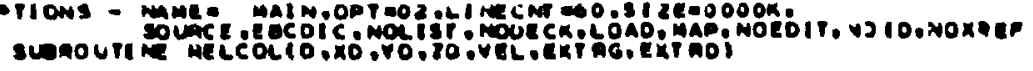

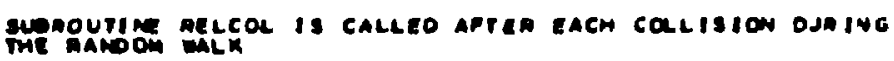

13v 0003

134 0004

134 0009

134 0006

isv 000 >

15v 0000

ISY :00:8

134 0012

134 0012

ISN 0014

13y :0015

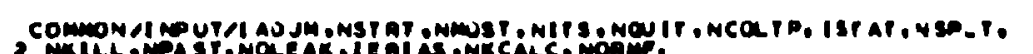

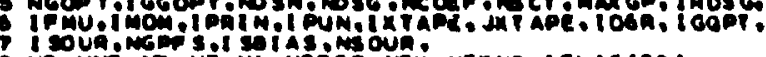

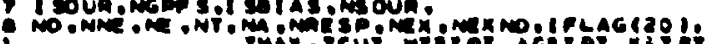

Ulmo y

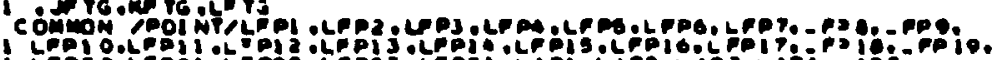

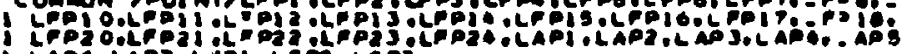

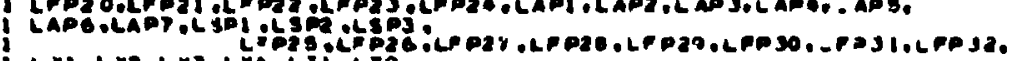

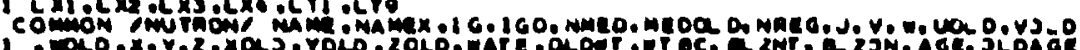

'cono;

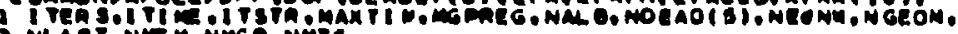

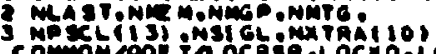

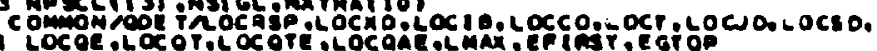

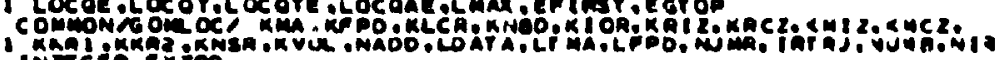

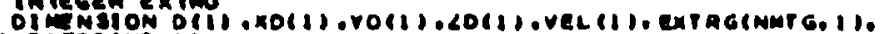

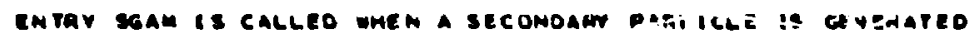

IFI 1G.ME . 2) AI TUAN

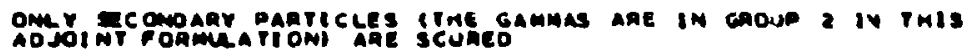

$00101=1.00$

DE REC TOO LOCATIONS ARE USEO TO IMOICATE IME DIAECT 13H

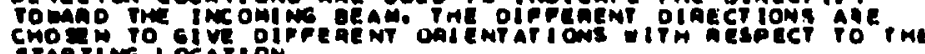

nution 


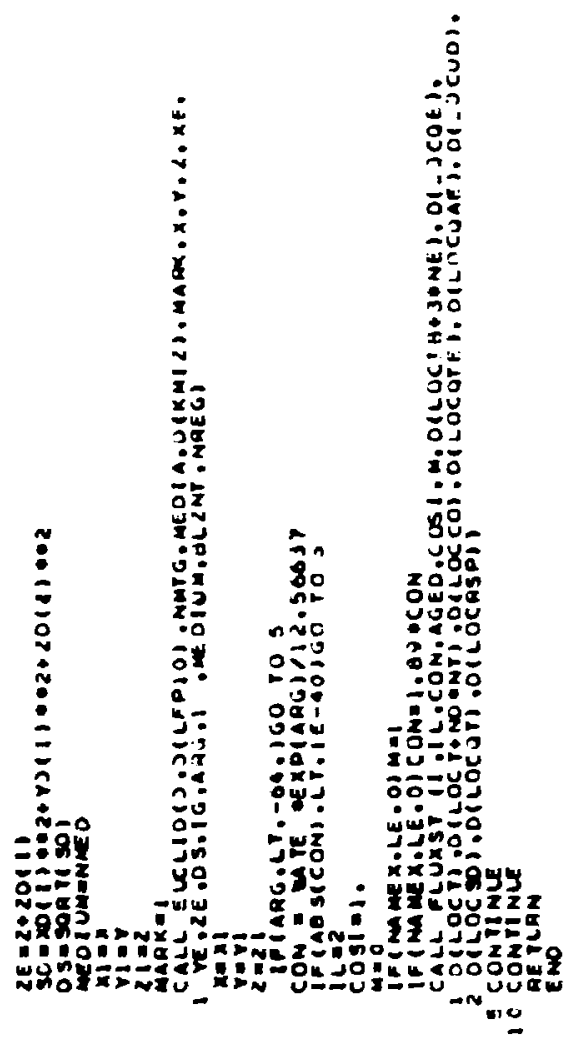

noop-nnen

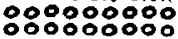

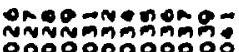
०0000\%0\%००००

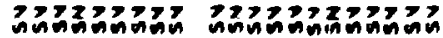



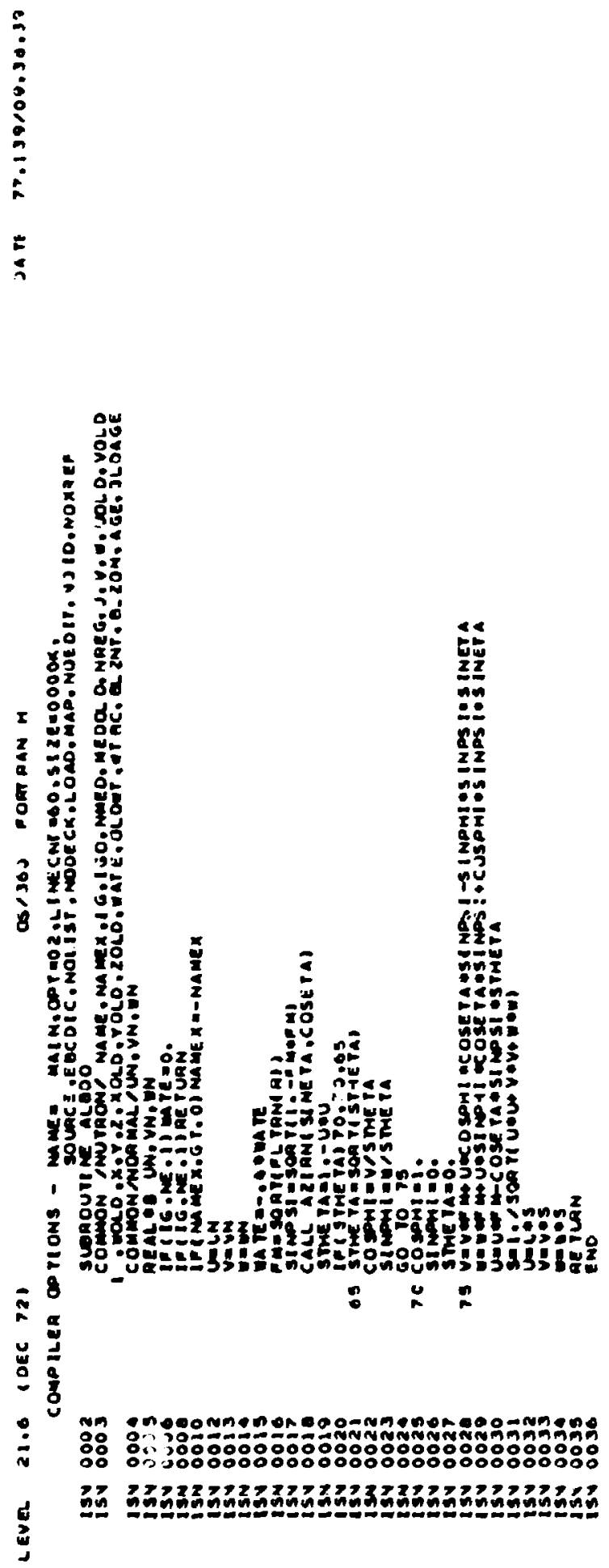
AHPX MODUE LOG ANO LIST OF CARJ INOUT DATA

modu L LAYA mas BEEN CALLEO

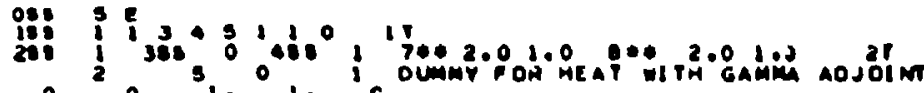

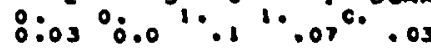

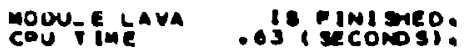

cou ine .03 iseconosit iro

rest of gamma mearing

28: $0,100,1000,100,100001000000$

3: I $10^{1000}, 0^{1} 0^{0}, 32,32$

$i_{2}, 1: 000: 111$

itigna reating test

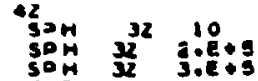

ENi⿺

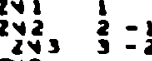

Nón.

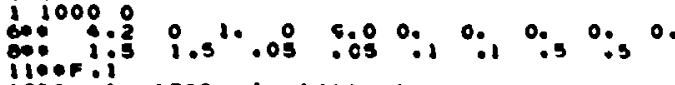

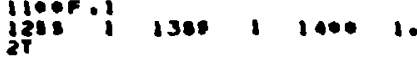

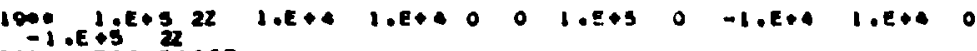

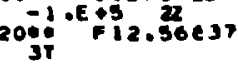

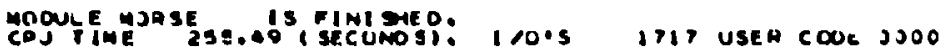

Srsten co0t 000 


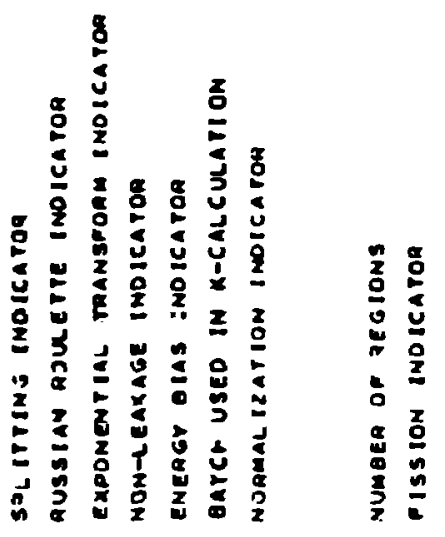

:

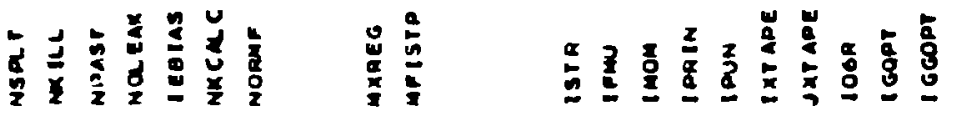

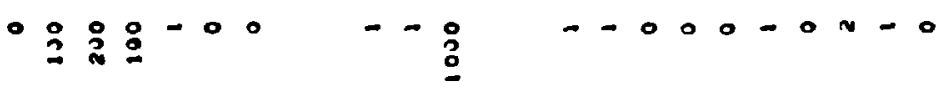

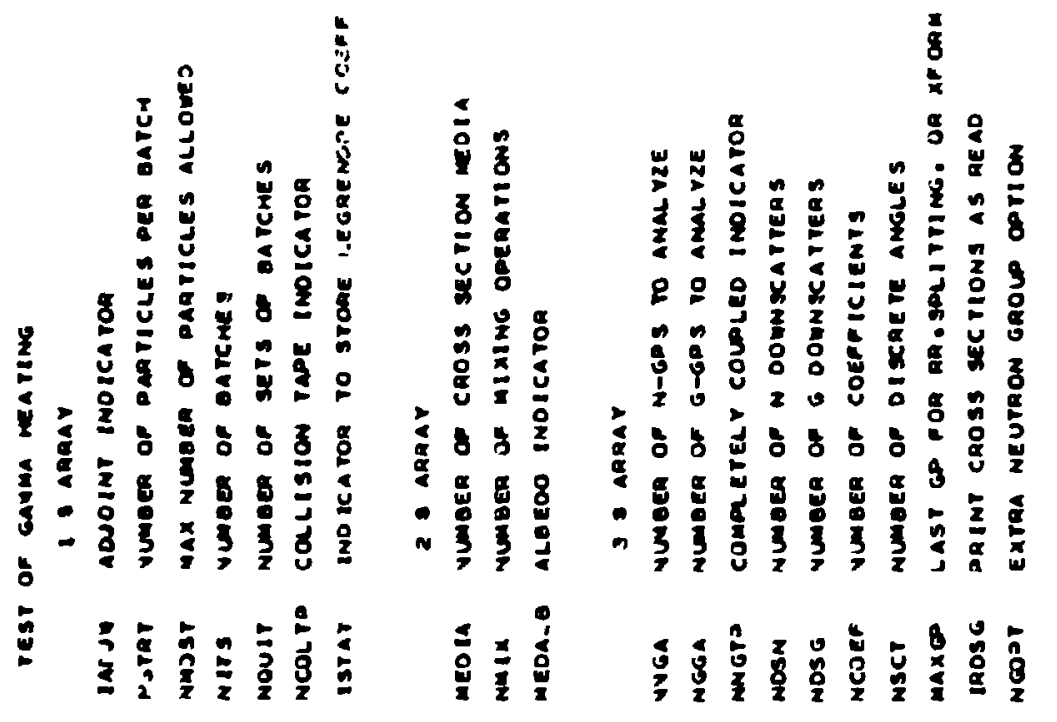




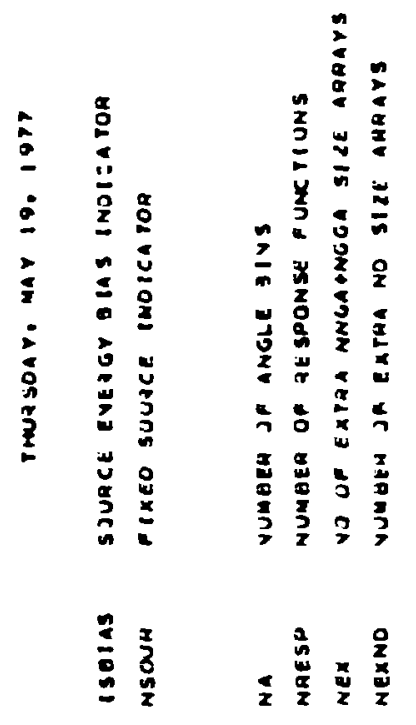

- noo

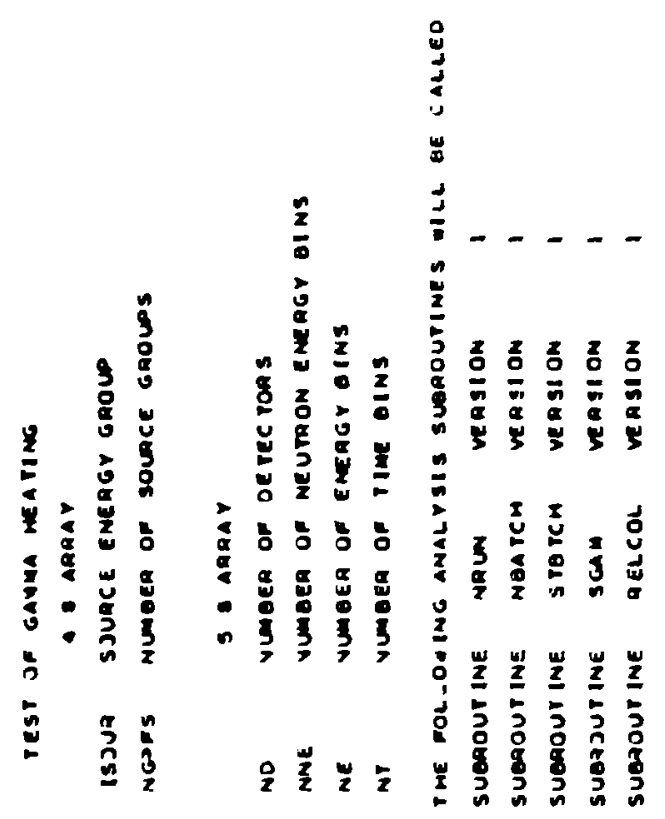




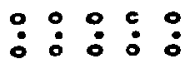
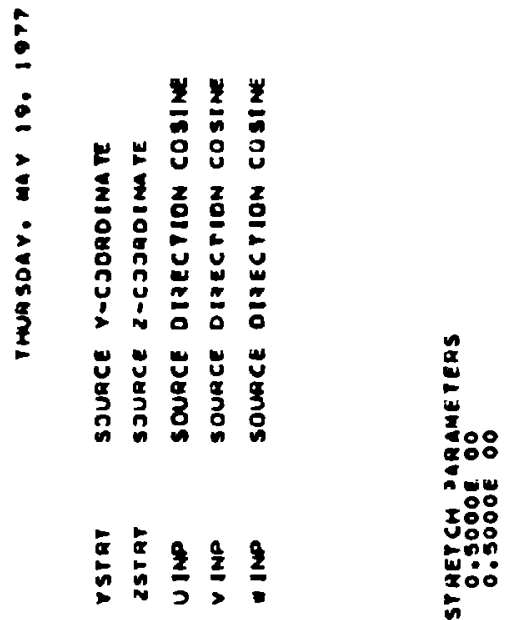

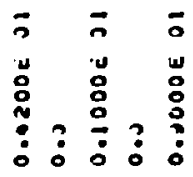

บับ

3용

势。

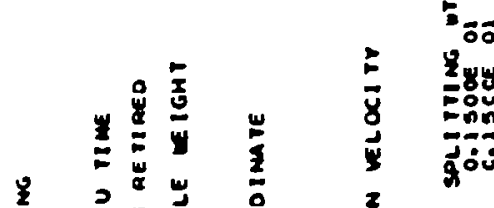

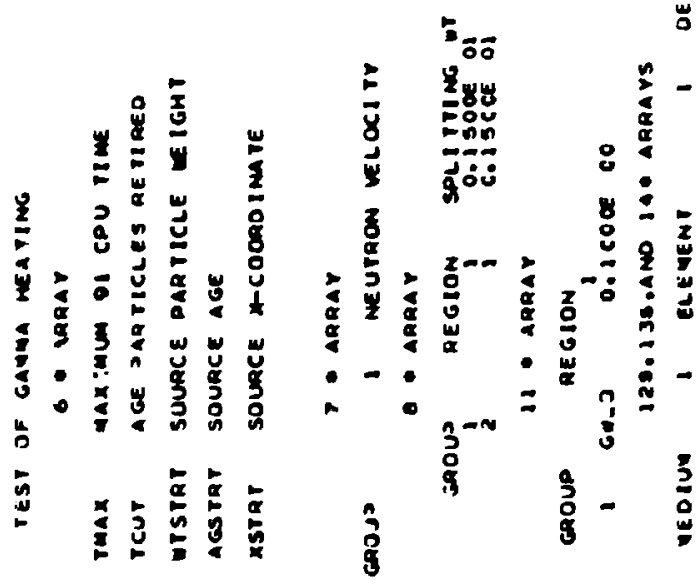


GaMma heATING TEST

IVOP $=0 \quad$ IOQG $=0$

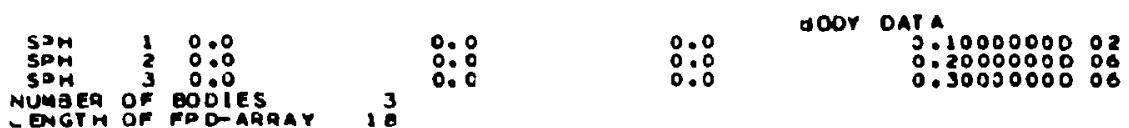

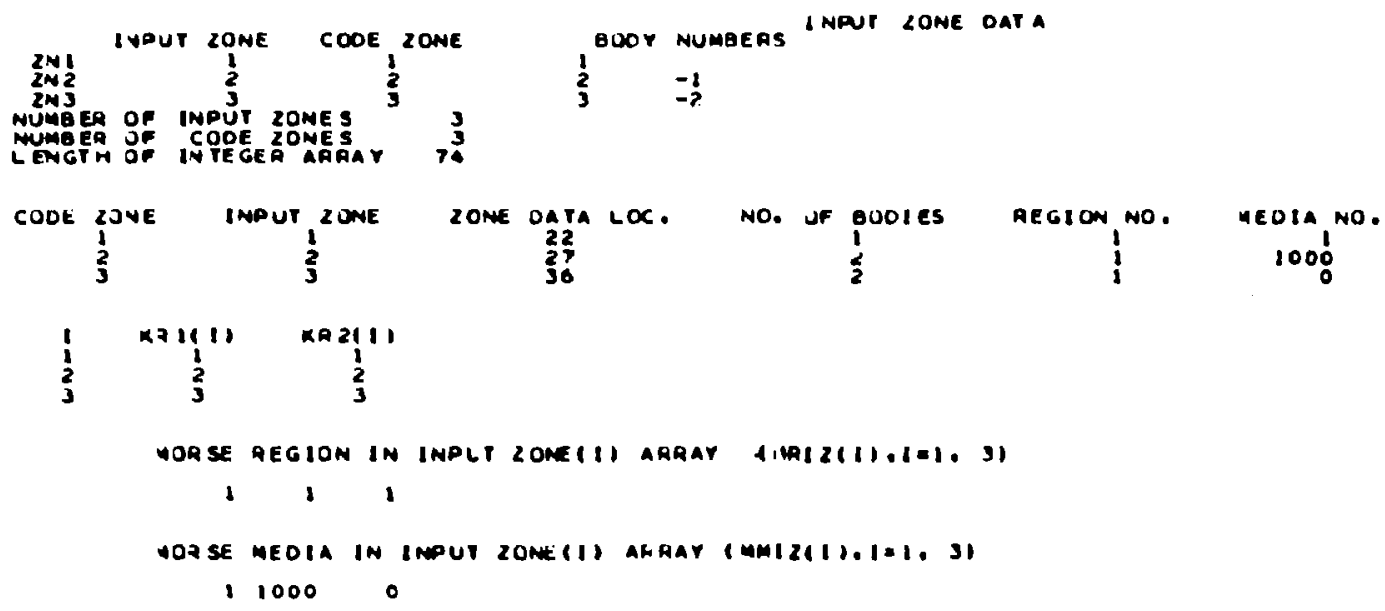


Test of gamma reating Detectios

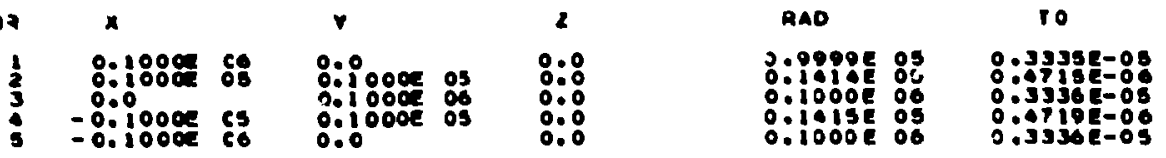

AESOJVE AUNCTIONS

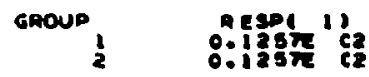

PIME Reoutaeo mor inout ans less man one secono.

- costiat oatch i

AANOOMNODOPIAFOAOSD

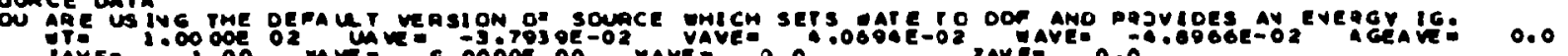

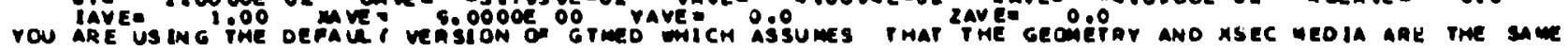

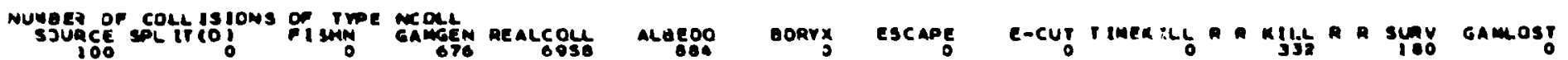

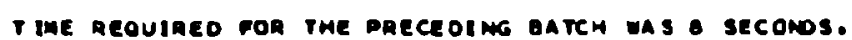

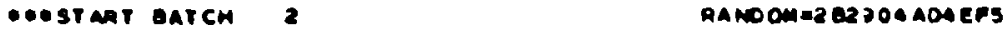

SOUPCE DATA

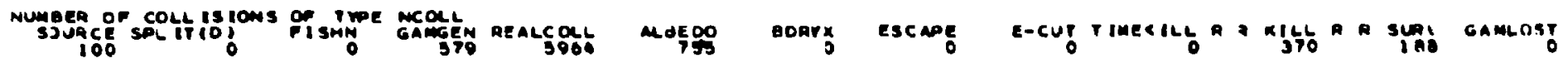

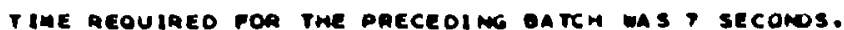

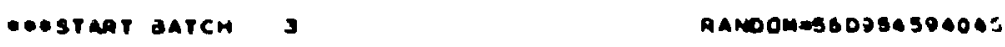

sovace dara

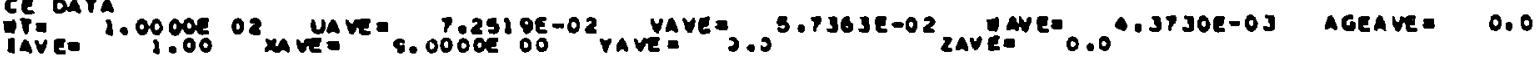

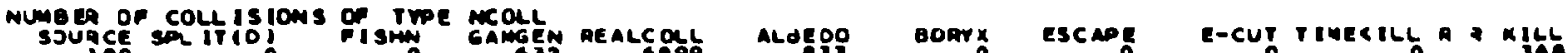

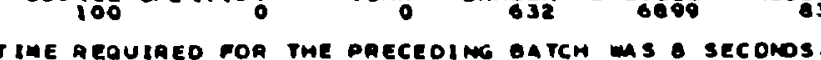

-onstapi batch a

Ranoomer 2302950001

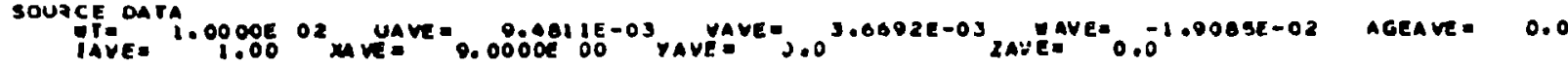

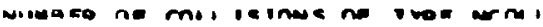



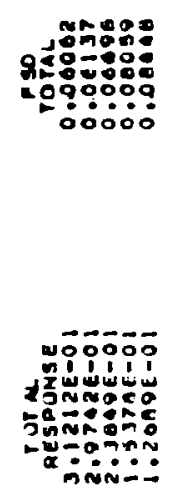